\title{
Effectiveness of Digital Interventions for Reducing Behavioral Risks of Cardiovascular Disease in Nonclinical Adult Populations: Systematic Review of Reviews
}

Natalie Gold ${ }^{1,2}$, DPhil; Amy Yau ${ }^{1,3}$, PhD; Benjamin Rigby ${ }^{1,4}$, MA; Chris Dyke ${ }^{1,5}$, MSc; Elizabeth Alice Remfry ${ }^{1,6}$, BSc; Tim Chadborn ${ }^{1}, \mathrm{PhD}$

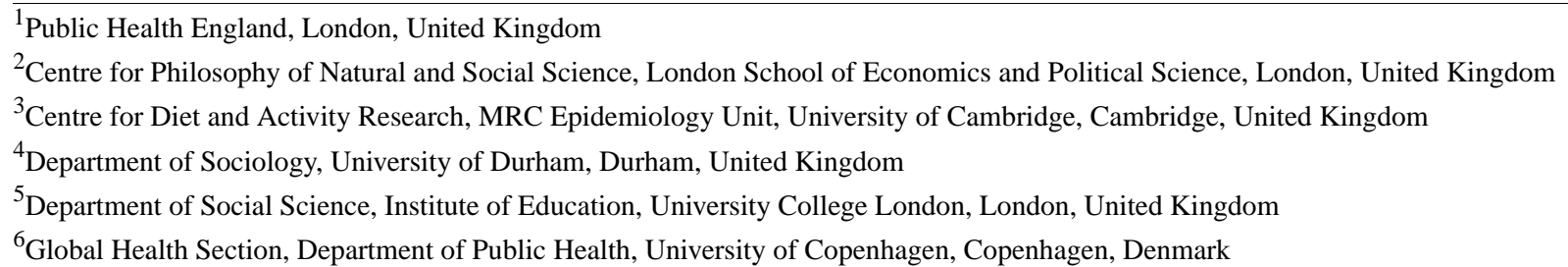

\section{Corresponding Author:}

Natalie Gold, DPhil

Public Health England

Wellington House

133-155 Waterloo Road

London, SE1 8UG

United Kingdom

Phone: 449767839044

Email: n.gold@1se.ac.uk

\section{Abstract}

Background: Digital health interventions are increasingly being used as a supplement or replacement for face-to-face services as a part of predictive prevention. They may be offered to those who are at high risk of cardiovascular disease and need to improve their diet, increase physical activity, stop smoking, or reduce alcohol consumption. Despite the popularity of these interventions, there is no overall summary and comparison of the effectiveness of different modes of delivery of a digital intervention to inform policy.

Objective: This review aims to summarize the effectiveness of digital interventions in improving behavioral and health outcomes related to physical activity, smoking, alcohol consumption, or diet in nonclinical adult populations and to identify the effectiveness of different modes of delivery of digital interventions.

Methods: We reviewed articles published in the English language between January 1, 2009, and February 25, 2019, that presented a systematic review with a narrative synthesis or meta-analysis of any study design examining digital intervention effectiveness; data related to adults ( $\geq 18$ years) in high-income countries; and data on behavioral or health outcomes related to diet, physical activity, smoking, or alcohol, alone or in any combination. Any time frame or comparator was considered eligible. We searched MEDLINE, Embase, PsycINFO, Cochrane Reviews, and gray literature. The AMSTAR-2 tool was used to assess review confidence ratings.

Results: We found 92 reviews from the academic literature (47 with meta-analyses) and 2 gray literature items (1 with a meta-analysis). Digital interventions were typically more effective than no intervention, but the effect sizes were small. Evidence on the effectiveness of digital interventions compared with face-to-face interventions was mixed. Most trials reported that intent-to-treat analysis and attrition rates were often high. Studies with long follow-up periods were scarce. However, we found that digital interventions may be effective for up to 6 months after the end of the intervention but that the effects dissipated by 12 months. There were small positive effects of digital interventions on smoking cessation and alcohol reduction; possible effectiveness in combined diet and physical activity interventions; no effectiveness for interventions targeting physical activity alone, except for when interventions were delivered by mobile phone, which had medium-sized effects; and no effectiveness observed for interventions targeting diet alone. Mobile interventions were particularly effective. Internet-based interventions were generally effective. 
Conclusions: Digital interventions have small positive effects on smoking, alcohol consumption, and in interventions that target a combination of diet and physical activity. Small effects may have been due to the low efficacy of treatment or due to nonadherence. In addition, our ability to make inferences from the literature we reviewed was limited as those interventions were heterogeneous, many reviews had critically low AMSTAR-2 ratings, analysis was typically intent-to-treat, and follow-up times were relatively short.

Trial Registration: PROSPERO International Prospective Register of Systematic Reviews CRD42019126074; https://www.crd.york.ac.uk/prospero/display_record.php?RecordID=126074.

(J Med Internet Res 2021;23(5):e19688) doi: 10.2196/19688

\section{KEYWORDS}

alcohol; behavior change; cardiovascular disease; diet; digital interventions; digital medicine; internet interventions; mHealth; mobile interventions; physical activity; smoking; tobacco; mobile phone

\section{Introduction}

\section{Background}

The National Health Service (NHS) Long Term Plan sets out the UK government's vision for preventing health problems and supporting the self-management of conditions [1]. A major target is cardiovascular disease (CVD), which causes $28 \%$ of all deaths in the United Kingdom and is the largest cause of premature death in deprived areas [2,3]. England's primary large-scale intervention for CVD prevention is the NHS Health Check program [1,2], which was introduced in 2009 [4]. It is one of the largest public health prevention programs in the world, with over 6 million people in England having a check between 2013 and 2018 [5]. The NHS Health Check is a CVD risk assessment, which should be offered every 5 years to all adults aged between 40 and 74 years with no pre-existing vascular condition. As a result, people with previously undiagnosed conditions can be put on a clinical pathway and those who are at risk of developing a condition can be offered lifestyle support and advice to manage their risk. In particular, cardiovascular risk can be reduced by modifying 4 types of behavior: diet [6], physical activity [7], smoking [8], and alcohol consumption [9].

A key pillar of the Long Term Plan is predictive prevention - the use of technology and digital tools to identify health risks, make early diagnoses, and support positive health behaviors of those most at need through targeted treatments [3]. Predictive prevention, including the NHS Health Check, involves risk communication and behavior change. Evidence shows that risk communication alone does not lead to behavior change [10-13]. Therefore, we need to support behavior change. Digital tools are an increasingly important part of that landscape, and digital behavior change interventions may be offered to people after their NHS Health Check to manage their risk by helping them modify their diet, physical activity, smoking, or alcohol consumption.

Digital tools may be used either to supplement face-to-face services or to replace them. Replacement is particularly germane, since there is anecdotal evidence that face-to-face services are increasingly being defunded. In addition, services may need to shift from face-to-face to digital in response to the COVID-19 pandemic, which occurred after we had completed the review. Providers may hope that digital tools will offer a low-cost solution, with the potential to reach more people than traditional face-to-face services; however, the research base needs to be evaluated to see if there is sufficient evidence [14].

\section{Aims and Objectives}

The first step is to establish whether digital interventions are effective. We also need to know which modes of delivery are most effective in order to allocate resources to develop the most promising digital tools or to know where research is needed, if the evidence base is lacking. In this systematic review of reviews, we aim to summarize the evidence on the effectiveness of digital interventions in improving dietary, physical activity, smoking, and alcohol consumption behaviors in nonpatient adult populations in high-income countries.

\section{Methods}

\section{Overview}

The study protocol was registered with PROSPERO (registration number: CRD42019126074). All deviations from the protocol are explained in the Methods section. We followed the PRISMA (Preferred Reporting Items for Systematic Reviews and Meta-Analyses) guidelines for reporting (see Multimedia Appendix 1 for the checklist) [15].

\section{Data Sources}

Relevant reviews were obtained through an internet-based search and a manual search. First, 4 internet-based databases (MEDLINE, Embase, PsycINFO, and Cochrane Reviews) were searched for peer-reviewed review articles published in English between January 1, 2009, and February 25, 2019. We limited our search dates, only starting in January 2009, to make our study manageable and also because we expected reviews published in the last decade to capture earlier papers. Publications were restricted to English due to the absence of translation expertise. Gray literature searches were conducted in OpenGrey, ProQuest Dissertations and Theses, Google, and targeted websites (see Multimedia Appendix 2 for search terms and the gray literature search strategy). More articles were identified by manual searches of the reference lists from excluded reviews of reviews. We did not search in study registries, as we were looking for systematic reviews. We did not conduct full hand searches or consult experts to ascertain the literature for pragmatic and logistical reasons. 


\section{Review Selection}

The reviews were screened using a three-stage process. A total of 2 reviewers (NG and AY) examined titles and discarded reviews that did not meet the inclusion criteria (Textbox 1). Each reviewer then independently screened the abstracts of $10 \%$ of the remaining reviews to identify studies that potentially met the inclusion criteria. Interreviewer agreement on inclusion was also assessed. Reviewers disagreed on 11 of 41 decisions. All disagreements were resolved through discussion. AY screened all the remaining abstracts. The relevant review articles were then obtained in full and screened independently for eligibility by NG and AY. Any disagreement over eligibility was resolved through discussion with a third reviewer (TC or BR).

Textbox 1. Inclusion and exclusion criteria.

Inclusion criteria

1. Study type: systematic reviews (whose reporting of the evidence could be either by narrative synthesis or by meta-analysis) that reported on the effectiveness of digital interventions in changing health-related behavior and/or health outcomes. We did not restrict by study design of the included studies within the systematic reviews.

2. Population: this included adult nonclinical populations. We aimed to assess the effectiveness of digital interventions in relation to CVD prevention relevant to the NHS Health Check program, which is offered to adults aged between 40 and 74 years (England, United Kingdom). Where populations were mixed, we included the review if the population of interest could be isolated. Where impossible to isolate the population of interest, reviews were included if $\geq 50 \%$ of the studies were of relevant populations.

3. Intervention: we included digital interventions targeting behaviors related to diet, physical activity, smoking, and/or alcohol consumption. Digital interventions include interventions delivered over the internet (web-based or websites), mobile telephone interventions (including texts and mobile apps), social media, computer-delivered interventions, and wearable technology. Interventions incorporating both digital and face-to-face components were also included.

4. Comparator: there were no restrictions. We extracted information about the comparators where available, allowing us to review effectiveness compared with both nondigital interventions and nonintervention controls.

5. Outcome: this included behavioral or health outcomes related to diet, physical activity, alcohol consumption, and smoking. Reviews that considered these areas of behavior, either individually or in combination, were included.

6. Time frame for follow-up: any time frame.

Exclusion criteria

1. Study type: reviews of reviews, conference abstracts, protocols, opinion pieces, and commentaries. We excluded reviews of reviews because we expected that most of the reviews gathered in a review of reviews would already be included in our study. Therefore, including reviews of reviews would have led to double counting of some information.

2. Population: reviews that only considered any of the following in $\geq 50 \%$ of included studies: children and adolescents, students, adults aged <40 years, pregnant women, management of existing CVD or other health conditions, and low- to middle-income countries. These criteria were selected to protect the ecological validity of this review, as relevant to the NHS Health Check.

3. Intervention: reviews of nondigital interventions. We did not consider television, radio, or telephone calls to be digital, as they are not often used in digital interventions for public health.

4. Comparator: no exclusion criteria.

5. Outcome: feasibility, acceptability, participation, and engagement only.

6. Superseded: this included reviews updated by subsequent reviews that included all the same studies as the original.

\section{Study Quality Assessment}

Review confidence was critically appraised independently by 2 reviewers (NG and AY) for a $10 \%$ subsample of the included publications, using the AMSTAR-2 tool [16]. The findings were discussed to check for consistency. The remaining articles were divided and assessed by NG or AY. Any uncertainty was resolved through discussion with a third reviewer (BR).

\section{Data Extraction}

Data were extracted using a standardized form (Multimedia Appendix 3). We extracted data on the following predefined review components: objective, population, inclusion and exclusion criteria, search date, included studies (number, type, and countries), follow-up, method of synthesis, results and findings, and comparator. A total of 2 reviewers (NG and AY) extracted the data independently for $10 \%$ of the publications.
Discrepancies were resolved through discussion. The remaining publications were divided among both reviewers. During data extraction, we also noted information about the control condition and any information on a comparison of effectiveness of no-intervention versus active controls. Data on adherence and attrition were also recorded, where available. The data extraction form has been presented in Multimedia Appendix 3.

\section{Analysis}

We conducted a systematic narrative synthesis using extracted data from included articles. No statistical analyses were conducted and meta-analysis was not possible with the included articles. We have presented results in the following categories: diet, physical activity, diet and physical activity combined, smoking, alcohol consumption, and multiple areas of behavior (all combinations other than diet and physical activity). Where there were enough reviews, we grouped by mode of delivery, 
especially internet (including email and interventions that require accessing a website) and mobile phone (including apps and SMS text messaging interventions); social media was categorized separately from internet, mainly because there are enough papers to make the subdivision worthwhile but also because the social aspect may differentiate social media interventions from other forms of internet interventions, so that it is appropriately considered a subclass [17]. When reporting effect sizes, for Cohen $d$, Hedges $g$, and other measures of standardized mean difference (SMD), we followed the convention that 0.2 is a small effect size, 0.5 is a medium effect size, and 0.8 is a large effect size [18]. For risk ratios (RRs), we classified 1.22 as small, 1.86 as medium, and 3.00 as large; for odds ratios (ORs), these were 1.32, 2.38, and 4.70, respectively [19].

\section{Results}

\section{Overview}

Searches identified 1739 potentially relevant records. After screening the titles and abstracts, 154 articles were retrieved in full. An additional 36 articles were identified through hand searches and gray literature searches. In total, 94 reviews met the inclusion criteria (Figure 1). A list of reviews excluded after full-text screening is provided in Multimedia Appendix 4. We were unable to retrieve one gray literature item by May 13, 2019, and it was therefore excluded.

Figure 1. PRISMA flowchart. PRISMA: Preferred Reporting Items for Systematic Reviews and Meta-Analyses.

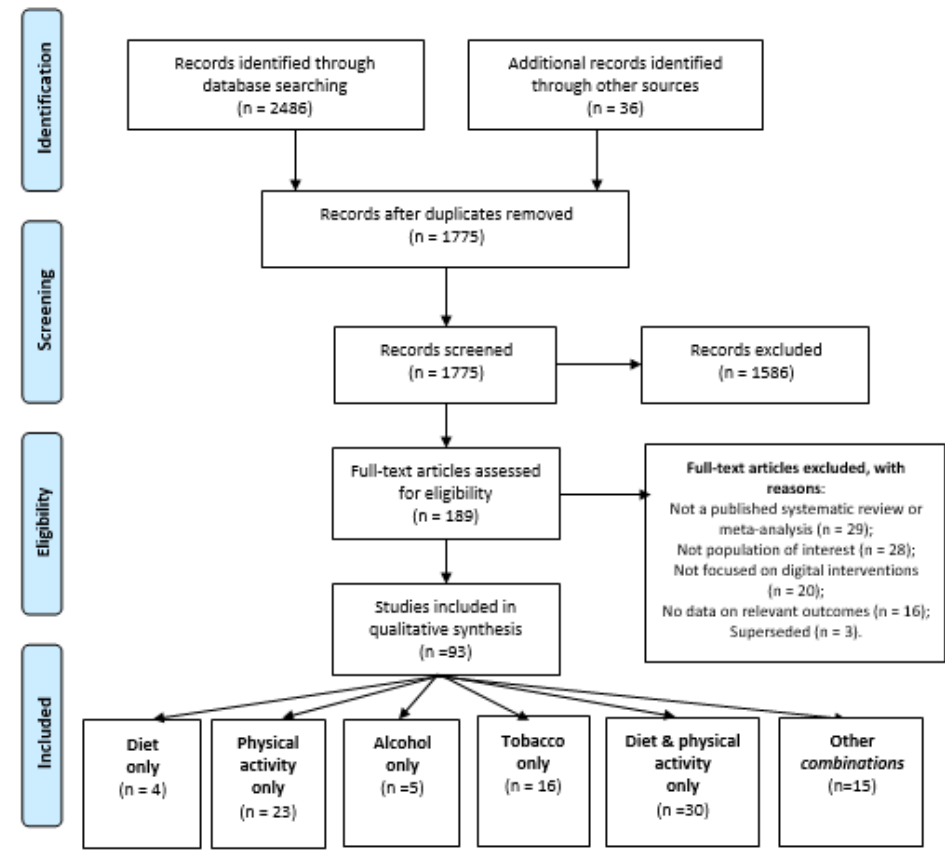

\section{Review Characteristics}

The included reviews examined the effectiveness of digital interventions on diet only [20-23], physical activity only [24-46], diet and physical activity combined [47-76] (for some it was possible to extract separate results about diet and physical activity behaviors, whereas some reported more general results on weight loss outcomes), alcohol consumption [77-82], and smoking cessation [83-98]. A further 15 reviews examined the effectiveness of digital interventions on a combination of our 4 target areas of behavior (not diet and physical activity; again, sometimes it was possible to extract separate information for each target area but other times, the results were only reported in combination) [99-113]. Some reviews covered a number of areas of behavior because their research questions focused on a health outcome (eg, CVD) or a mode of intervention delivery (eg, internet interventions) rather than a behavior. Where extracting information on an area of behavior from a combination review was possible, we included the relevant data in the results for that area. For a breakdown of the reviews, with the number found in each area, for each mode of intervention and type of control see Multimedia Appendix 5.

The populations reviewed were general (nonclinical) adult populations. However, the diet and physical activity reviews were often restricted to populations of individuals with overweight or obesity. The alcohol reviews were often restricted to problem drinkers, defined with reference to local guidelines $[77,79,80]$, questionnaire scores [81], or reduced productivity at work [78]. There was a range of modes of delivery, including mobile app, SMS text messaging, social media, pedometer, wearable, and interactive computer program. The reviews included both active and nonactive or minimal intervention controls, many pooling both types, but where possible, we tried to extract separate information about effectiveness of active compared with nonactive controls. We regarded the provision of educational materials as a nonactive or minimal control. More reviews included behavioral outcomes (such as fat consumption, fruit and vegetable consumption, physical activity, alcohol consumption, smoking cessation, and smoking abstinence) than health outcomes (such as weight loss, BMI, and waist 
circumference). Multimedia Appendix 6 provides the key characteristics of all included studies.

\section{Review Confidence Ratings}

The confidence rating of each review is presented in the study characteristics table in Multimedia Appendix 6, and a summary of confidence ratings is provided in Table $1 ; 84 \%$ (79/93) of reviews were rated as critically low. During the completion of the AMSTAR-2 tool, reviewers noted that most reviews failed to satisfy items 4 (including and justifying a publication language inclusion criterion) and 7 (providing a list of excluded reviews and justifications). Both items are considered critical for systematic reviews but not for meta-analyses. A modified rating was produced alongside the original AMSTAR-2 rating, which did not classify either of these flaws as critical, to see whether a variation among reviews would be revealed. However, little change was observed, with only 5 reviews moving from a rating of critically low to low.

Table 1. Risk of bias: a summary of AMSTAR-2 confidence ratings.

\begin{tabular}{lllll}
\hline Category & \multicolumn{2}{l}{ Confidence rating (modified rating) } & & Cow \\
& High & Moderate & $1(1)$ & $3(3)$ \\
\hline Diet & $0(0)$ & $0(0)$ & $0(1)$ & $20(19)$ \\
Physical activity & $0(0)$ & $1(1)$ & $1(3)$ & $28(26)$ \\
Diet and physical activity low & $1(1)$ & $1(1)$ & $4(4)$ \\
Alcohol & $0(0)$ & $0(0)$ & $4(4)$ & $10(10)$ \\
Smoking & $0(0)$ & $2(2)$ & $2(4)$ & $14(12)$ \\
Other & $0(0)$ & $1(1)$ & $9(14)$ & $79(74)$ \\
Total & $0(0)$ & $5(5)$ & &
\end{tabular}

\section{Effectiveness of Interventions}

Table 2 summarizes the effectiveness of the different types of interventions. 
Table 2. Summary of the effectiveness of different types of interventions.

\begin{tabular}{|c|c|c|c|c|c|c|}
\hline \multirow{2}{*}{$\begin{array}{l}\text { Find- } \\
\text { ings }\end{array}$} & \multicolumn{6}{|c|}{ Behavioral categories } \\
\hline & Diet & Physical activity & Diet and physical Activity & Smoking & Alcohol & $\begin{array}{l}\text { Other combina- } \\
\text { tions }\end{array}$ \\
\hline $\begin{array}{l}\text { Number } \\
\text { of re- } \\
\text { views }\end{array}$ & $\begin{array}{l}20 \text { reviews, of } \\
\text { which } 4 \text { were } \\
\text { diet-only }\end{array}$ & $\begin{array}{l}\text { - } 45 \text { reviews, of } \\
\text { which } 22 \text { were } \\
\text { physical activity- } \\
\text { only }\end{array}$ & $\begin{array}{l}35 \text { reviews, of which } \\
29 \text { were on weight loss, } \\
\text { specifically combining } \\
\text { diet and physical activ- } \\
\text { ity, and } 3 \text { where results } \\
\text { on weight loss were } \\
\text { extractable from other } \\
\text { combinations }\end{array}$ & $\begin{array}{l}28 \text { reviews, of } \\
\text { which } 16 \text { were } \\
\text { smoking-only }\end{array}$ & $\begin{array}{l}13 \text { reviews, of } \\
\text { which } 6 \text { were } \\
\text { alcohol-only }\end{array}$ & 11 \\
\hline $\begin{array}{l}\text { Effec- } \\
\text { tiveness } \\
\text { com- } \\
\text { pared } \\
\text { with } \\
\text { mixed } \\
\text { (active } \\
\text { and non- } \\
\text { active) } \\
\text { controls }\end{array}$ & $\begin{array}{l}\text { Small effect } \\
\text { of internet in- } \\
\text { terventions on } \\
\text { pooled behav- } \\
\text { iors but het- } \\
\text { erogenous } \\
\text { outcome mea- } \\
\text { sures and ef- } \\
\text { fect sizes } \\
\text { Some small } \\
\text { effects of mo- } \\
\text { bile phone in- } \\
\text { terventions on } \\
\text { behavior, but } \\
\text { no effects on } \\
\text { weight loss } \\
\text { Mixed effects } \\
\text { of social me- } \\
\text { dia but no ef- } \\
\text { fects on } \\
\text { weight loss } \\
\text { Mixed effects } \\
\text { of computer- } \\
\text { delivered in- } \\
\text { terventions, } \\
\text { and positive } \\
\text { effects were } \\
\text { not clinically } \\
\text { significant }\end{array}$ & $\begin{array}{l}\text { Mixed evidence } \\
\text { for the effect of } \\
\text { internet interven- } \\
\text { tions on physical } \\
\text { activity; where } \\
\text { there were effects, } \\
\text { they were small } \\
\text { and there was het- } \\
\text { erogeneity be- } \\
\text { tween studies } \\
\text { Mixed effects of } \\
\text { social media, with } \\
\text { meta-analyses not } \\
\text { finding significant } \\
\text { effects } \\
\text { Better evidence } \\
\text { for effects of mo- } \\
\text { bile phone inter- } \\
\text { ventions, with } \\
\text { most studies in } \\
\text { narrative reviews } \\
\text { showing effective- } \\
\text { ness, with effect } \\
\text { There for SMS text } \\
\text { able evidence } \\
\text { from reviews as- } \\
\text { sessing a variety } \\
\text { or a combination } \\
\text { of interventions } \\
\text { ventions ranging } \\
\text { from small to } \\
\text { medium } \\
\text { Mixed evidence } \\
\text { for the effect of } \\
\text { exergaming } \\
\text { Mixed evidence } \\
\text { on computer-deliv- } \\
\text { ered interventions } \\
\text { (2 reviews); the } \\
\text { meta-analysis } \\
\text { found small ef- } \\
\text { fects }\end{array}$ & $\begin{array}{l}\text { Positive effects of inter- } \\
\text { net interventions for } \\
\text { weight loss and BMI, } \\
\text { especially when part of } \\
\text { blended interventions } \\
\text { - } \quad \text { Mixed effects of social } \\
\text { media interventions } \\
\text { Medium-sized effects } \\
\text { of mobile interventions } \\
\text { on weight loss } \\
\text { Mixed results from re- } \\
\text { views covering a vari- } \\
\text { ety of interventions, } \\
\text { around half of the inter- } \\
\text { ventions were found to } \\
\text { be effective }\end{array}$ & $\begin{array}{l}\text { Small positive } \\
\text { effects for inter- } \\
\text { net, mobile, and } \\
\text { computer-deliv- } \\
\text { ered interven- } \\
\text { tions. Mixed evi- } \\
\text { dence about inter- } \\
\text { ventions from re- } \\
\text { views that cov- } \\
\text { ered multiple } \\
\text { types of digital } \\
\text { technologies }\end{array}$ & $\begin{array}{l}\text { Small and } \\
\text { medium posi- } \\
\text { tive effects }\end{array}$ & $\begin{array}{l}\text { Small positive ef- } \\
\text { fects on behavior } \\
\text { and on health out- } \\
\text { comes for internet, } \\
\text { computer-deliv- } \\
\text { ered, SMS text } \\
\text { messaging, and } \\
\text { prompts delivered } \\
\text { by all methods; } \\
\text { however, results } \\
\text { were not favorable } \\
\text { for apps }\end{array}$ \\
\hline
\end{tabular}




\begin{tabular}{|c|c|c|c|c|c|c|}
\hline \multirow{2}{*}{$\begin{array}{l}\text { Find- } \\
\text { ings }\end{array}$} & \multicolumn{6}{|c|}{ Behavioral categories } \\
\hline & Diet & Physical activity & Diet and physical Activity & Smoking & Alcohol & $\begin{array}{l}\text { Other combina- } \\
\text { tions }\end{array}$ \\
\hline $\begin{array}{l}\text { Compar- } \\
\text { ison to } \\
\text { no or } \\
\text { minimal } \\
\text { interven- } \\
\text { tion }\end{array}$ & $\begin{array}{l}\text { Little evidence } \\
\text { available and it } \\
\text { was mixed: } \\
\text { - } \quad \text { One meta- } \\
\text { analysis of } \\
\text { voice-re- } \\
\text { sponse inter- } \\
\text { ventions } \\
\text { found no ef- } \\
\text { fect } \\
\text { One meta- } \\
\text { analysis of } \\
\text { computer-tai- } \\
\text { lored interven- } \\
\text { tions found a } \\
\text { small effect }\end{array}$ & $\begin{array}{l}\text { Mixed results: } \\
\text { - } \quad \text { Mixed results for } \\
\text { internet and mo- } \\
\text { bile interventions } \\
\text { - } \text { One review of so- } \\
\text { cial media inter- } \\
\text { ventions, finding } \\
\text { increased in steps } \\
\text { taken but not ener- } \\
\text { gy expenditure, to- } \\
\text { tal physical activi- } \\
\text { ty, or moderate-to- } \\
\text { vigorous physical } \\
\text { activity } \\
\text { Small positive ef- } \\
\text { fect of interactive } \\
\text { voice response and } \\
\text { computer-deliv- } \\
\text { ered interventions }\end{array}$ & $\begin{array}{l}\text { - More effective than } \\
\text { minimal controls for } \\
\text { internet and computer- } \\
\text { based interventions, } \\
\text { but effect sizes were } \\
\text { small }\end{array}$ & $\begin{array}{l}\text { Small positive } \\
\text { effects for inter- } \\
\text { net-based inter- } \\
\text { ventions and for } \\
\text { digital interven- } \\
\text { tions not broken } \\
\text { down by mode } \\
\text { of delivery), mo- } \\
\text { bile interventions } \\
\text { more effective } \\
\text { than completely } \\
\text { passive controls } \\
\text { and as effective } \\
\text { as minimal con- } \\
\text { trols }\end{array}$ & $\begin{array}{l}\text { Effective com- } \\
\text { pared with no- } \\
\text { intervention } \\
\text { controls }\end{array}$ & $\begin{array}{l}\text { Effective com- } \\
\text { pared with no-inter- } \\
\text { vention controls, } \\
\text { with small effects } \\
\text { for internet and } \\
\text { medium effects for } \\
\text { SMS text messag- } \\
\text { ing interventions }\end{array}$ \\
\hline $\begin{array}{l}\text { Compar- } \\
\text { ison } \\
\text { with ac- } \\
\text { tive con- } \\
\text { trols }\end{array}$ & $\begin{array}{l}\text { Mixed; in nar- } \\
\text { rative synthe- } \\
\text { ses (of inter- } \\
\text { net, mobile, } \\
\text { and combina- } \\
\text { tions of inter- } \\
\text { ventions), less } \\
\text { than 50\% of } \\
\text { studies in } \\
\text { each review } \\
\text { found signifi- } \\
\text { cant improve- } \\
\text { ments }\end{array}$ & $\begin{array}{l}\text { Increase in physi- } \\
\text { cal activity for in- } \\
\text { ternet-based inter- } \\
\text { ventions } \\
\text { - Mixed results for } \\
\text { mobile interven- } \\
\text { tions } \\
\text { Promising results } \\
\text { about wearables }\end{array}$ & $\begin{array}{l}\text { - Internet-based interven- } \\
\text { tions not more effec- } \\
\text { tive than active con- } \\
\text { trols, unless as a part of } \\
\text { blended interventions } \\
\text { - Computer-delivered } \\
\text { interventions led to less } \\
\text { weight loss than in- } \\
\text { person treatment }\end{array}$ & $\begin{array}{l}\text { No evidence that } \\
\text { digital interven- } \\
\text { tions are any } \\
\text { more effective } \\
\text { than active con- } \\
\text { trols }\end{array}$ & $\begin{array}{l}\text { No evidence } \\
\text { that digital inter- } \\
\text { ventions are any } \\
\text { more effective } \\
\text { than active con- } \\
\text { trols; mixed evi- } \\
\text { dence about } \\
\text { whether active } \\
\text { controls are } \\
\text { more effective } \\
\text { than digital }\end{array}$ & $\begin{array}{l}\text { Very small effects } \\
\text { for internet and } \\
\text { small effects for } \\
\text { computer-delivered } \\
\text { and SMS text mes- } \\
\text { saging interven- } \\
\text { tions }\end{array}$ \\
\hline $\begin{array}{l}\text { Sustain- } \\
\text { ability }\end{array}$ & $\begin{array}{l}\text { Relatively } \\
\text { few studies } \\
\text { with follow- } \\
\text { ups } \\
\text { Digital inter- } \\
\text { ventions were } \\
\text { largely effec- } \\
\text { tive over a 3- } \\
\text { to 6-month } \\
\text { period } \\
\text { Evidence was } \\
\text { more mixed } \\
\text { in the long } \\
\text { term (>12 } \\
\text { months) }\end{array}$ & $\begin{array}{l}\text { Relatively few } \\
\text { studies with fol- } \\
\text { low-ups } \\
\text { Some evidence for } \\
\text { sustainability of } \\
\text { internet and combi- } \\
\text { nations of digital } \\
\text { interventions at 6- } \\
\text { month follow-up } \\
\text { Some evidence } \\
\text { that the effect of } \\
\text { mobile interven- } \\
\text { tions is short lived }\end{array}$ & $\begin{array}{l}\text { Few studies, but those } \\
\text { found that effective- } \\
\text { ness declined over time }\end{array}$ & & $\begin{array}{l}\text { Mixed results } \\
\text { about whether } \\
\text { the effect was } \\
\text { sustained. No } \\
\text { follow-ups ex- } \\
\text { ceeded } 12 \\
\text { months. Evi- } \\
\text { dence suggests } \\
\text { effects have di- } \\
\text { minished by } \\
\text { this point }\end{array}$ & $\begin{array}{l}\text { Mixed evidence on } \\
\text { when the effect } \\
\text { size peaks (short or } \\
\text { medium term); ef- } \\
\text { fects decrease but } \\
\text { still exist at } 12 \\
\text { months }\end{array}$ \\
\hline
\end{tabular}




\begin{tabular}{|c|c|c|c|c|c|c|}
\hline \multirow{2}{*}{$\begin{array}{l}\text { Find- } \\
\text { ings }\end{array}$} & \multicolumn{6}{|c|}{ Behavioral categories } \\
\hline & Diet & Physical activity & Diet and physical Activity & Smoking & Alcohol & $\begin{array}{l}\text { Other combina- } \\
\text { tions }\end{array}$ \\
\hline & & & & $\begin{array}{r}\text { Mixed ev } \\
\text { - } \quad \text { Effe } \\
\text { net- } \\
\text { vent } \\
\text { taine } \\
\text { mon } \\
\text { is di } \\
\text { abou } \\
\text { effec } \\
\text { taine } \\
\text { mon } \\
\text { Mix } \\
\text { on th } \\
\text { abili } \\
\text { inter } \\
\text { No e } \\
\text { susta } \\
\text { com } \\
\text { ered } \\
\text { tions } \\
\text { Revi } \\
\text { veye } \\
\text { digit } \\
\text { tions } \\
\text { ferer } \\
\text { mod } \\
\text { foun } \\
\text { effec } \\
\text { the } 1 \\
\text { follo }\end{array}$ & & \\
\hline
\end{tabular}

\section{Diet}

\section{Review Characteristics}

A total of 20 reviews reported findings on diet behaviors or a weight loss outcome that resulted from an intervention that only targeted diet. The breakdown of their characteristics is shown in Table S1.
Effectiveness of Digital Interventions on Diet Compared With Mixed (Active and Nonactive) Controls for Diet Behaviors

The effectiveness of digital interventions in improving results related to diet was at best mixed, for both behavioral and health outcomes (Table 3); where improvements were reported, the effect sizes were typically small. This was the case across all modes of delivery. 
Table 3. Results of reviews on diet, ordered by type of control and mode of delivery of intervention.

\begin{tabular}{|c|c|c|c|c|c|c|c|}
\hline Review & $\begin{array}{l}\text { Relevant } \\
\text { studies, } \mathrm{n} \\
\text { (total stud- } \\
\text { ies) }\end{array}$ & $\begin{array}{l}\text { Method of synthe- } \\
\text { sis }\end{array}$ & Interventions & $\begin{array}{l}\text { Dietary out- } \\
\text { comes }\end{array}$ & Follow-up & Summary of findings & $\begin{array}{l}\text { AMSTAR- } \\
2 \text { rating }\end{array}$ \\
\hline \multicolumn{8}{|c|}{ Mixed (active and nonactive) controls } \\
\hline $\begin{array}{l}\text { Afshin et al } \\
\text { (2016) [99] }\end{array}$ & $20(224)$ & $\begin{array}{l}\text { Narrative synthe- } \\
\text { sis of RCTs }{ }^{\mathrm{a}} \text { and } \\
\text { quasiexperimen- } \\
\text { tal studies }\end{array}$ & $\begin{array}{l}\text { Internet and } \\
\text { mobile }\end{array}$ & $\begin{array}{l}\text { Various di- } \\
\text { etary behav- } \\
\text { iors, fruit and } \\
\text { veg intake }\end{array}$ & $\begin{array}{l}1 \text { week-2 } \\
\text { years }\end{array}$ & $\begin{array}{l}70 \%(14 / 20) \text { studies found } \\
\text { significant dietary improve- } \\
\text { ments. Effect sizes varied due } \\
\text { to heterogeneity in dietary } \\
\text { targets. The intake of fruit in- } \\
\text { creased by approximately } \\
1 \text { serving/day. Two RCTs as- } \\
\text { sessed mobile - based inter- } \\
\text { ventions and fruit or vegetable } \\
\text { intake; each found significant } \\
\text { improvement (by } 2 \text { and } \\
4 \text { servings/day) }\end{array}$ & $\begin{array}{l}\text { Critically } \\
\text { low }\end{array}$ \\
\hline $\begin{array}{l}\text { Aneni et al } \\
\text { (2014) [100] }\end{array}$ & $9(29)$ & $\begin{array}{l}\text { Narrative synthe- } \\
\text { sis of RCTs }\end{array}$ & Internet & Dietary intake & 6-24 months & $\begin{array}{l}44 \%(4 / 9) \text { high-quality studies } \\
\text { demonstrated improvements } \\
\text { in diet }\end{array}$ & $\begin{array}{l}\text { Critically } \\
\text { low }\end{array}$ \\
\hline $\begin{array}{l}\text { Hou et al } \\
\text { (2013) [108] }\end{array}$ & $7(38)$ & $\begin{array}{l}\text { Narrative synthe- } \\
\text { sis of studies with } \\
\text { comparison or } \\
\text { control groups }\end{array}$ & Internet & $\begin{array}{l}\text { Dietary intake } \\
\text { and fruit and } \\
\text { vegetable in- } \\
\text { take }\end{array}$ & Not reported & $\begin{array}{l}\text { All the studies examining nu- } \\
\text { trition alone and nutrition and } \\
\text { other factors in addition to } \\
\text { PA }^{\mathrm{b}} \text { reported increases in } \\
\text { healthy dietary behaviors in } \\
\text { the intervention groups. Inter- } \\
\text { ventions showed promising } \\
\text { effects at } 12 \text { months for reduc- } \\
\text { tions in body fat, weight, and } \\
\text { dietary fat intake }\end{array}$ & $\begin{array}{l}\text { Critically } \\
\text { low }\end{array}$ \\
\hline $\begin{array}{l}\text { Lustria et al } \\
\text { (2013) [110] }\end{array}$ & $10(40)$ & $\begin{array}{l}\text { Meta-analysis of } \\
\text { experimental and } \\
\text { quasiexperimen- } \\
\text { tal studies }\end{array}$ & $\begin{array}{l}\text { Internet-tailored } \\
\text { interventions }\end{array}$ & $\begin{array}{l}\text { Fruit and veg- } \\
\text { etable intake } \\
\text { and saturated } \\
\text { fat intake }\end{array}$ & $\begin{array}{l}6 \text { weeks- } 6 \\
\text { months }\end{array}$ & $\begin{array}{l}\text { Small effect sizes, Cohen } \\
d=0.223(\mathrm{k}=4 ; 95 \% \text { CI } 0.11 \\
\text { to } 0.33 ; P<.001)\end{array}$ & $\begin{array}{l}\text { Critically } \\
\text { low }\end{array}$ \\
\hline $\begin{array}{l}\text { Maon et al } \\
\text { (2012) [63] }\end{array}$ & $8(26)$ & $\begin{array}{l}\text { Narrative synthe- } \\
\text { sis of RCTs }\end{array}$ & Internet & $\begin{array}{l}\text { Fruit and veg- } \\
\text { etable con- } \\
\text { sumption }\end{array}$ & $\begin{array}{l}6 \text { weeks-2 } \\
\text { years }\end{array}$ & $\begin{array}{l}50 \%(4 / 8) \text { studies investigat- } \\
\text { ing healthy eating habits re- } \\
\text { ported positive changes such } \\
\text { as increased fruit or vegetable } \\
\text { consumption }\end{array}$ & $\begin{array}{l}\text { Critically } \\
\text { low }\end{array}$ \\
\hline $\begin{array}{l}\text { Webb et al } \\
\text { (2010) [113] }\end{array}$ & $10(85)$ & $\begin{array}{l}\text { Meta-analysis of } \\
\text { RCTs }\end{array}$ & Internet & $\begin{array}{l}\text { Dietary behav- } \\
\text { ior }\end{array}$ & Not reported & $\begin{array}{l}\text { Small effect sizes on behavior } \\
\text { were observed for interven- } \\
\text { tions that targeted only dietary } \\
\text { behavior (Cohen } d_{+}=0.20 \text {; } \\
\mathrm{k}=10 ; 95 \% \text { CI } 0.02 \text { to } 0.37 \text { ) }\end{array}$ & $\begin{array}{l}\text { Critically } \\
\text { low }\end{array}$ \\
\hline $\begin{array}{l}\text { DiFillipo et al } \\
\text { (2015) [21] }\end{array}$ & $3(3)$ & $\begin{array}{l}\text { Narrative synthe- } \\
\text { sis of RCTs }\end{array}$ & Mobile & Weight loss & $\begin{array}{l}8 \text { weeks- } 6 \\
\text { months }\end{array}$ & $\begin{array}{l}100 \%(3 / 3) \text { of studies found } \\
\text { a numerical tendency to } \\
\text { weight loss compared with the } \\
\text { control; only } 33 \%(1 / 3) \text { of } \\
\text { studies was statistically signif- } \\
\text { icant; this study showed an } \\
\text { increase in self-monitoring }\end{array}$ & $\begin{array}{l}\text { Critically } \\
\text { low }\end{array}$ \\
\hline $\begin{array}{l}\text { McCarroll et al } \\
\text { (2017) [23] (di- } \\
\text { et only) }\end{array}$ & $21(23)$ & $\begin{array}{l}\text { Narrative synthe- } \\
\text { sis of RCTs }\end{array}$ & Mobile & $\begin{array}{l}\text { Healthy eating } \\
\text { and weight } \\
\text { loss (diet on- } \\
\text { ly) }\end{array}$ & $1-24$ months & $\begin{array}{l}\text { Small positive effects of inter- } \\
\text { ventions on healthy eating } \\
\text { ( } 5 / 8,63 \% \text { of trials) and } \\
\text { weight loss ( } 5 / 13,38 \% \text { of tri- } \\
\text { als), but studies were judged } \\
\text { to be of poor quality }\end{array}$ & $\begin{array}{l}\text { Critically } \\
\text { low }\end{array}$ \\
\hline
\end{tabular}




\begin{tabular}{|c|c|c|c|c|c|c|c|}
\hline Review & $\begin{array}{l}\text { Relevant } \\
\text { studies, n } \\
\text { (total stud- } \\
\text { ies) }\end{array}$ & $\begin{array}{l}\text { Method of synthe- } \\
\text { sis }\end{array}$ & Interventions & $\begin{array}{l}\text { Dietary out- } \\
\text { comes }\end{array}$ & Follow-up & Summary of findings & $\begin{array}{l}\text { AMSTAR- } \\
2 \text { rating }\end{array}$ \\
\hline $\begin{array}{l}\text { Lyzwinksi et al } \\
(2014)[60]\end{array}$ & $5(14)$ & $\begin{array}{l}\text { Meta-analysis of } \\
\text { RCTs }\end{array}$ & Mobile & $\begin{array}{l}\text { Fruit and veg } \\
\text { intake, energy } \\
\text { density }\end{array}$ & $\begin{array}{l}8 \text { weeks-12 } \\
\text { months }\end{array}$ & $\begin{array}{l}100 \%(3 / 3) \text { of studies found } \\
\text { an improvement in fruit and } \\
\text { vegetable intake; } 100 \%(2 / 2) \\
\text { studies found an improvement } \\
\text { in energy density and eating } \\
\text { behavior }\end{array}$ & $\begin{array}{l}\text { Critically } \\
\text { low }\end{array}$ \\
\hline $\begin{array}{l}\text { Palmer et al } \\
(2018)[111]\end{array}$ & $3(71)$ & $\begin{array}{l}\text { Meta-analysis of } \\
\text { RCTs }\end{array}$ & Mobile & $\begin{array}{l}\text { Saturated fat } \\
\text { intake, BMI, } \\
\text { salt intake, } \\
\text { weight from } \\
\text { diet-only inter- } \\
\text { ventions }\end{array}$ & Not reported & $\begin{array}{l}2(100 \%) \text { studies showed no } \\
\text { effect of interventions on } \\
\text { weight or dietary intake }\end{array}$ & Moderate \\
\hline $\begin{array}{l}\text { Elaheebocus et } \\
\text { al (2018) [56] }\end{array}$ & $20(134)$ & $\begin{array}{l}\text { Narrative synthe- } \\
\text { sis of RCTs }\end{array}$ & Social media & Body weight & 6-48 months & $\begin{array}{l}75 \%(15 / 20) \text { of studies using } \\
\text { web-based social networks } \\
\text { had positive results for dietary } \\
\text { outcomes }\end{array}$ & $\begin{array}{l}\text { Critically } \\
\text { low }\end{array}$ \\
\hline $\begin{array}{l}\text { Mita et al } \\
(2016) \text { [65] }\end{array}$ & $12(16)$ & $\begin{array}{l}\text { Meta-analysis of } \\
\text { RCTs }\end{array}$ & Social media & $\begin{array}{l}\text { Body weight } \\
\text { and fruit and } \\
\text { vegetable in- } \\
\text { take }\end{array}$ & Not reported & $\begin{array}{l}\text { No significant differences } \\
\left(\mathrm{SMD}^{\mathrm{c}}-0.14 ; 95 \% \mathrm{CI}-0.28\right. \\
\text { to } 0.01 \text { ), with similar findings } \\
\text { for body weight (SMD } 0.07 \text {; } \\
95 \% \mathrm{CI}-0.17 \text { to } 0.20 \text { ) and } \\
\text { fruit and vegetable intake } \\
\text { (SMD } 0.39 ; 95 \% \mathrm{CI}-0.11 \text { to } \\
0.89 \text { ) }\end{array}$ & Moderate \\
\hline $\begin{array}{l}\text { Williams et al } \\
\text { (2012) [75] }\end{array}$ & $5(22)$ & $\begin{array}{l}\text { Meta-analysis of } \\
\text { RCTs }\end{array}$ & Social media & $\begin{array}{l}\text { Body weight } \\
\text { and dietary fat }\end{array}$ & 3-24 months & $\begin{array}{l}\text { No significant differences in } \\
\text { changes in weight (SMD } 0 ; \\
95 \% \text { CI }-0.19 \text { to } 0.19 ; 10 \\
\text { studies) however, pooled re- } \\
\text { sults from } 5 \text { studies showed a } \\
\text { significant decrease in dietary } \\
\text { fat consumption with social } \\
\text { media (SMD }-0.35 ; 95 \% \text { CI } \\
-0.68 \text { to }-0.02)\end{array}$ & $\begin{array}{l}\text { Critically } \\
\text { low }\end{array}$ \\
\hline $\begin{array}{l}\text { Wieland et al } \\
\text { (2012) [74] }\end{array}$ & $18(18)$ & $\begin{array}{l}\text { Meta-analysis of } \\
\text { RCTs, quasi- } \\
\text { RCTs, cluster } \\
\text { RCTs, and quasi- } \\
\text { experimental } \\
\text { studies }\end{array}$ & $\begin{array}{l}\text { Interactive, } \\
\text { computer-based }\end{array}$ & $\begin{array}{l}\text { Fat intake, } \\
\text { calorie intake, } \\
\text { total fiber, } \\
\text { fruit and veg- } \\
\text { etable intake; } \\
\text { weight, BMI, } \\
\text { and waist cir- } \\
\text { cumference }\end{array}$ & $\begin{array}{l}4 \text { weeks-30 } \\
\text { months }\end{array}$ & $\begin{array}{l}\text { Computer-based interventions } \\
\text { led to greater weight loss than } \\
\text { minimal interventions (MD } \\
-1.5 \mathrm{~kg} \text {; } 95 \% \mathrm{CI}-2.1 \text { to }-0.9 \text { ) } \\
\text { but less weight loss than in- } \\
\text { person treatment (MD } 2.1 \mathrm{~kg} \text {; } \\
95 \% \text { CI } 0.8 \text { to } 3.4 \text {; one trial); } \\
\text { in the } 3 \text { - to 6-month follow- } \\
\text { up, there was a significant de- } \\
\text { crease in percentage calories } \\
\text { from fat (MD - } 1.1 \% \text { ), and } \\
\text { improved fiber intake (dietary } \\
\text { fiber score MD } 1.3 \text { ); there } \\
\text { was no significant effect on } \\
\text { energy intake at } 30 \text { months }\end{array}$ & $\begin{array}{l}\text { Critically } \\
\text { low }\end{array}$ \\
\hline
\end{tabular}




\begin{tabular}{|c|c|c|c|c|c|c|c|}
\hline Review & $\begin{array}{l}\text { Relevant } \\
\text { studies, n } \\
\text { (total stud- } \\
\text { ies) }\end{array}$ & $\begin{array}{l}\text { Method of synthe- } \\
\text { sis }\end{array}$ & Interventions & $\begin{array}{l}\text { Dietary out- } \\
\text { comes }\end{array}$ & Follow-up & Summary of findings & $\begin{array}{l}\text { AMSTAR- } \\
2 \text { rating }\end{array}$ \\
\hline $\begin{array}{l}\text { Harris et al } \\
\text { (2011) [22] (di- } \\
\text { et only) }\end{array}$ & $25(43)$ & $\begin{array}{l}\text { Meta-analysis of } \\
\text { RCTs }\end{array}$ & $\begin{array}{l}\text { Interactive, } \\
\text { computer based }\end{array}$ & $\begin{array}{l}\text { Fat intake, } \\
\text { fruit and veg- } \\
\text { etable con- } \\
\text { sumption, } \\
\text { fiber intake, } \\
\text { and energy in- } \\
\text { take }\end{array}$ & 0-12 months & $\begin{array}{l}\text { Interventions did not produce } \\
\text { clinically significant changes } \\
\text { in dietary behavior: fruit and } \\
\text { vegetable intake had a } \\
\text { weighted MD of } 0.24 \text { servings } \\
\text { per day ( } 95 \% \text { CI } 0.04 \text { to } 0.44 \\
\text { servings; } P=.02) \text {, total energy } \\
\text { consumed from fat had a } \\
\text { weighted MD of }-1.4 \%(95 \% \\
\text { CI }-2.5 \% \text { to }-0.3 \% ; P=.01) \text {; } \\
\text { there were no significant } \\
\text { weighted MDs in intake of } \\
\text { total fat, saturated fat, daily } \\
\text { dietary fiber, or daily energy; } \\
\text { there were no significant ef- } \\
\text { fects on fruit and vegetable } \\
\text { consumption and BMI at } 12 \\
\text { months }\end{array}$ & Low \\
\hline $\begin{array}{l}\text { Carvalho de } \\
\text { Menzes et al } \\
(2016)[53]\end{array}$ & $15(18)$ & $\begin{array}{l}\text { Narrative synthe- } \\
\text { sis of all designs }\end{array}$ & Various & $\begin{array}{l}\text { Fat consump- } \\
\text { tion and fruit } \\
\text { and vegetable } \\
\text { consumption }\end{array}$ & 1-36 months & $\begin{array}{l}\text { Among the changes in eating } \\
\text { habits, statistically significant } \\
\text { reductions were observed in } \\
\text { the consumption of fat (total } \\
\text { fat, saturated fat, and transfat), } \\
\text { and there was an increase in } \\
\text { the ingestion of fruit and veg- } \\
\text { etables }\end{array}$ & $\begin{array}{l}\text { Critically } \\
\text { low }\end{array}$ \\
\hline \multicolumn{8}{|l|}{ Nonactive controls } \\
\hline $\begin{array}{l}\text { Krebs et al } \\
(2010) \text { [109] }\end{array}$ & $51(76)$ & $\begin{array}{l}\text { Meta-analysis of } \\
\text { RCTs }\end{array}$ & $\begin{array}{l}\text { Computer-tai- } \\
\text { lored }\end{array}$ & $\begin{array}{l}\text { Dietary intake } \\
\text { of fat, vegeta- } \\
\text { bles, and fruits }\end{array}$ & 1-24 months & $\begin{array}{l}\text { The mean effect size for di- } \\
\text { etary fat reduction was } g=0.22 \\
\text { ( } 95 \% \text { CI } 0.18 \text { to } 0.26 \text { ); mean } \\
\text { effect size for fruit and veg- } \\
\text { etable intake was } g=0.16 \\
\text { ( } 95 \% \text { CI } 0.10 \text { to } 0.21 \text { ). }\end{array}$ & Low \\
\hline $\begin{array}{l}\text { Tsoli et al } \\
(2018) \text { [73] }\end{array}$ & $2(15)$ & $\begin{array}{l}\text { Meta-analysis of } \\
\text { RCTs }\end{array}$ & $\begin{array}{l}\text { Interactive } \\
\text { voice response }\end{array}$ & Diet & $\begin{array}{l}6 \text { weeks-12 } \\
\text { months }\end{array}$ & $\begin{array}{l}\text { No statistically significant ef- } \\
\text { fect on behaviors related to } \\
\text { diet (Hedges } g=0.130 ; 95 \% \\
\text { CI }-0.088 \text { to } 0.347 ; \mathrm{k}=2 \text {; } \\
P=.24 \text { ) }\end{array}$ & $\begin{array}{l}\text { Critically } \\
\text { low }\end{array}$ \\
\hline \multicolumn{8}{|l|}{ Active controls } \\
\hline $\begin{array}{l}\text { Burke et al } \\
\text { (2011) [20] (di- } \\
\text { et only) }\end{array}$ & $5(24)$ & $\begin{array}{l}\text { Narrative synthe- } \\
\text { sis of RCTs and } \\
\text { descriptive stud- } \\
\text { ies }\end{array}$ & $\begin{array}{l}\text { Personal digital } \\
\text { assistants }\end{array}$ & $\begin{array}{l}\text { Weight loss } \\
\text { (diet only) }\end{array}$ & 3-24 months & $\begin{array}{l}\text { In all studies, self-monitoring } \\
\text { was significantly associated } \\
\text { with weight loss, but there } \\
\text { was minimal evidence to sup- } \\
\text { port the use of personal digital } \\
\text { assistants over other methods } \\
\text { of self-monitoring; they may } \\
\text { result in long-term reduced } \\
\text { risk of weight regain after } 18 \\
\text { months }\end{array}$ & $\begin{array}{l}\text { Critically } \\
\text { low }\end{array}$ \\
\hline
\end{tabular}




\begin{tabular}{|c|c|c|c|c|c|c|c|}
\hline Review & $\begin{array}{l}\text { Relevant } \\
\text { studies, n } \\
\text { (total stud- } \\
\text { ies) }\end{array}$ & $\begin{array}{l}\text { Method of synthe- } \\
\text { sis }\end{array}$ & Interventions & $\begin{array}{l}\text { Dietary out- } \\
\text { comes }\end{array}$ & Follow-up & Summary of findings & $\begin{array}{l}\text { AMSTAR- } \\
2 \text { rating }\end{array}$ \\
\hline $\begin{array}{l}\text { Covolo et al } \\
(2017)[104]\end{array}$ & $18(40)$ & $\begin{array}{l}\text { Narrative synthe- } \\
\text { sis of RCTs }\end{array}$ & Mobile & $\begin{array}{l}\text { BMI and waist } \\
\text { circumfer- } \\
\text { ence; fruit and } \\
\text { vegetable in- } \\
\text { take, and } \\
\text { high-sugar } \\
\text { food intake }\end{array}$ & $\begin{array}{l}6 \text { months-2 } \\
\text { years }\end{array}$ & $\begin{array}{l}56 \%(10 / 18) \text { of RCTs found } \\
\text { no difference between inter- } \\
\text { vention and control group; } \\
33 \%(6 / 18) \text { studies showed a } \\
\text { significant increase in the } \\
\text { consumption of fruit and veg- } \\
\text { etables and reduced sugar- } \\
\text { sweetened beverage consump- } \\
\text { tion }\end{array}$ & $\begin{array}{l}\text { Critically } \\
\text { low }\end{array}$ \\
\hline
\end{tabular}

${ }^{\mathrm{a}} \mathrm{RCT}$ : randomized controlled trial.

${ }^{\mathrm{b}} \mathrm{PA}$ : physical activity.

${ }^{\mathrm{c}} \mathrm{SMD}$ : standardized mean difference.

${ }^{\mathrm{d}} \mathrm{MD}$ : mean difference.

Of the 6 reviews covering internet interventions, 2 meta-analyses found small, favorable effects of internet interventions on dietary behavior, Cohen $d=0.223$ [110] and Cohen $d+=0.20$ [113]. A total of 4 narrative syntheses found mixed results with heterogeneous target outcomes [63,99,100,108]. The most common dietary target across the studies was fruit intake, which increased by approximately 1 serving per day [99].

Across 6 reviews of mobile interventions, there was some evidence of positive effects on dietary behaviors, especially fruit and vegetable intake, but no statistically significant effects on weight loss $[21,23,60,99,104,111]$. One of these reviews [99] reported a previous systematic review (not covered in this paper) that found no significant change in calorie intake or the consumption of sugar-sweetened beverages in 4 trials evaluating diet.

A total of 3 social media reviews had mixed findings on dietary outcomes, such as fruit and vegetable intake and fat intake, but the 2 reviews with meta-analyses found no differences in weight $[65,75]$.

The effects of interactive computer interventions were equivocal and not clinically significant [22,74].

\section{Effect of Digital Interventions on Diet Compared With Nonactive Controls}

There was little evidence about the effectiveness of digital interventions on diet compared with no intervention or minimal intervention controls, and the available evidence was mixed (Table 3). One meta-analysis found small effects on fruit and vegetable intake $(g=0.16)$ and dietary fat reduction $(g=0.22)$ [109], but another meta-analysis found no statistically significant effects on dietary behaviors [73].

\section{Effect of Digital Interventions on Diet Compared With Active Controls}

The relative effectiveness of digital interventions on diet compared with active comparators was mixed, with

approximately half of the studies or less in narrative syntheses, showing that digital interventions were effective compared with active controls (Table 3). This was true across all modes of delivery: internet [99], mobile [99,104], and combined interventions [99].

\section{Sustainability of Effects on Diet at Follow-Up}

Reviews included studies that ranged from single-contact interventions to 5-year follow-ups (Table 3). Relatively few reviews reported on follow-ups; of those that did, about half reported follow-ups in the medium term (3-6 months) and half in the long term ( $\geq 12$ months).

Where reported, digital interventions were generally found to be effective for over 3 to 6 months. Several reviews have found positive results at 6 months $[20,21,74]$. However, this finding is not universal [111].

Long-term findings were more mixed in the 5 reviews that investigated them. Two reviews suggested promising effects at 12 months [108] and 18 months [20]. However, 3 reviews found no significant effects at 12 months [22], 24 months [111], and 30 months [74].

\section{Physical Activity}

\section{Review Characteristics}

We included the findings on physical activity-related outcomes from 45 systematic reviews. The breakdown of their characteristics is shown in Multimedia Appendix 6.

\section{Effectiveness of Digital Interventions on Physical Activity Compared With Mixed (Active and Nonactive) Controls}

The effectiveness of digital interventions was mixed across all modes of delivery, apart from mobiles, for which the evidence was consistently positive (Table 4). 
Table 4. Results of reviews on physical activity, ordered by type of control and further ordered by mode of delivery of intervention.

\begin{tabular}{|c|c|c|c|c|c|c|c|}
\hline Review & $\begin{array}{l}\text { Relevant } \\
\text { studies, } \mathrm{n} \\
\text { (total } \\
\text { studies) }\end{array}$ & Method of synthesis & Interventions & Outcomes & $\begin{array}{l}\text { Follow- } \\
\text { up }\end{array}$ & Summary of findings & $\begin{array}{l}\text { AMSTAR- } \\
2 \text { rating }\end{array}$ \\
\hline \multicolumn{8}{|c|}{ Mixed controls (active and nonactive) } \\
\hline $\begin{array}{l}\text { Aalbers et al } \\
(2011) \text { [47] }\end{array}$ & $2(10)$ & $\begin{array}{l}\text { Narrative synthesis } \\
\text { of randomized and } \\
\text { nonrandomized pre- } \\
\text { post controlled trials }\end{array}$ & Internet & $\begin{array}{l}\text { Total } \mathrm{PA}^{\mathrm{a}} \text { and MV- } \\
\mathrm{PA}^{\mathrm{b}}\end{array}$ & $\begin{array}{l}1.5-6.5 \\
\text { months }\end{array}$ & $\begin{array}{l}2 \text { studies reported the effects of } \\
\text { digital interventions, one was } \\
\text { less effective on MVPA than a } \\
\text { nonactive control and the other } \\
\text { demonstrated a small positive } \\
\text { effect on total } \mathrm{PA}^{\mathrm{b}}(P=.001) \text {. }\end{array}$ & $\begin{array}{l}\text { Critically } \\
\text { low }\end{array}$ \\
\hline $\begin{array}{l}\text { Aneni et al } \\
\text { (2014) [100] }\end{array}$ & $9(29)$ & $\begin{array}{l}\text { Narrative synthesis } \\
\text { of RCTs }^{c}\end{array}$ & Internet & PA measures & $\begin{array}{l}6-24 \\
\text { months }\end{array}$ & $\begin{array}{l}\text { No improvement was seen in } \\
\text { virtually all the studies with PA } \\
\text { outcome, only } 11 \%(1 / 9) \text { of } \\
\text { studies demonstrated a signifi- } \\
\text { cant intervention effect on PA. }\end{array}$ & $\begin{array}{l}\text { Critically } \\
\text { low }\end{array}$ \\
\hline $\begin{array}{l}\text { Bottorf et al } \\
\text { (2014) [26] } \\
\text { (PA only) }\end{array}$ & $8(35)$ & $\begin{array}{l}\text { Narrative synthesis } \\
\text { of all study types }\end{array}$ & Internet & $\begin{array}{l}\text { Changes in PA; step } \\
\text { count; self-reported } \\
\text { walking; BMI; waist } \\
\text { circumference; } \\
\text { weight }\end{array}$ & $\begin{array}{l}3-12 \\
\text { months }\end{array}$ & $\begin{array}{l}63 \%(5 / 8) \text { of studies demonstrat- } \\
\text { ed that PA significantly in- } \\
\text { creased in the internet-based } \\
\text { interventions, } 2 \text { studies showed } \\
\text { a nonsignificant difference, and } \\
\text { one showed that the effects } \\
\text { were indeterminable. }\end{array}$ & $\begin{array}{l}\text { Critically } \\
\text { low }\end{array}$ \\
\hline $\begin{array}{l}\text { Davies et al } \\
\text { (2012) [29] } \\
\text { (PA only) }\end{array}$ & $34(34)$ & $\begin{array}{l}\text { Meta-analysis of ex- } \\
\text { perimental design } \\
\text { studies }\end{array}$ & Internet & $\mathrm{PA}$ & $\begin{array}{l}2-52 \\
\text { weeks }\end{array}$ & $\begin{array}{l}\text { The estimated overall mean ef- } \\
\text { fect of internet-delivered inter- } \\
\text { ventions on PA was Cohen } \\
d=0.14(P<.001) \text {. Homogeneity } \\
\text { tests from the fixed-effect } \\
\text { analysis revealed significant } \\
\text { heterogeneity across studies } \\
(Q=73.75 ; P<.001) \text {. The overall } \\
\text { mean effect for sustained PA at } \\
\text { least } 6 \text { months postintervention } \\
(\mathrm{n}=11) \text { resulted in a small but } \\
\text { significant effect size Cohen } \\
d=0.11(P<.01) \text {. }\end{array}$ & $\begin{array}{l}\text { Critically } \\
\text { low }\end{array}$ \\
\hline $\begin{array}{l}\text { George et al } \\
\text { (2012) [33] } \\
\text { (PA only) }\end{array}$ & $2(14)$ & $\begin{array}{l}\text { Narrative synthesis } \\
\text { of all study types }\end{array}$ & Internet & $\begin{array}{l}\text { Step count; health } \\
\text { status; BMI; weekly } \\
\text { PA }\end{array}$ & $\begin{array}{l}2-8 \\
\text { months }\end{array}$ & $\begin{array}{l}\text { Increase in PA in } 100 \%(2 / 2) \\
\text { of online interventions where } \\
\text { participants were in competitive } \\
\text { teams, including one that } \\
\text { showed an increase in step } \\
\text { count. Poor quality evidence. }\end{array}$ & $\begin{array}{l}\text { Critically } \\
\text { low }\end{array}$ \\
\hline $\begin{array}{l}\text { Hou et al } \\
\text { (2013) [108] }\end{array}$ & $7(38)$ & $\begin{array}{l}\text { Narrative synthesis } \\
\text { of trials with compar- } \\
\text { ison or control group }\end{array}$ & Internet & $\begin{array}{l}\text { Level of physical } \\
\text { activity }\end{array}$ & $\begin{array}{l}0-12 \\
\text { months }\end{array}$ & $\begin{array}{l}86 \%(6 / 7) \text { of interventions were } \\
\text { successful in the studies focus- } \\
\text { ing primarily on PA. }\end{array}$ & $\begin{array}{l}\text { Critically } \\
\text { low }\end{array}$ \\
\hline $\begin{array}{l}\text { Jahangiry et } \\
\text { al (2017) } \\
\text { [35] (PA on- } \\
\text { ly) }\end{array}$ & $21(22)$ & $\begin{array}{l}\text { Meta-analysis of } \\
\text { controlled trials }\end{array}$ & Internet & $\begin{array}{l}\text { MVPA; walking; } \\
\text { step count (pedome- } \\
\text { ter) }\end{array}$ & $\begin{array}{l}1-20 \\
\text { weeks }\end{array}$ & $\begin{array}{l}36 \%(5 / 14) \text { of rials reporting } \\
\text { MVPA, } 50 \%(3 / 6) \text { of trials re- } \\
\text { porting step count, and } 29 \% \\
(4 / 14) \text { of studies reporting } \\
\text { minutes walking showed signif- } \\
\text { icant increases. The interven- } \\
\text { tions were influenced by the } \\
\text { age of participants and trial } \\
\text { length. }\end{array}$ & $\begin{array}{l}\text { Critically } \\
\text { low }\end{array}$ \\
\hline $\begin{array}{l}\text { Lustria et al } \\
\text { (2013) [110] }\end{array}$ & $12(40)$ & $\begin{array}{l}\text { Meta-analysis of ex- } \\
\text { perimental and } \\
\text { quasiexperimental } \\
\text { studies }\end{array}$ & Internet & Levels of PA & $\begin{array}{l}4 \\
\text { weeks- } \\
24 \\
\text { months }\end{array}$ & $\begin{array}{l}\text { The sample size-weighted } \\
\text { mean effect size for studies on } \\
\text { PA was not significant Cohen } \\
d=0.059(\mathrm{k}=12 ; 95 \% \mathrm{CI}-0.02 \\
\text { to } 0.14 \text { ). }\end{array}$ & $\begin{array}{l}\text { Critically } \\
\text { low }\end{array}$ \\
\hline
\end{tabular}




\begin{tabular}{|c|c|c|c|c|c|c|c|}
\hline Review & $\begin{array}{l}\text { Relevant } \\
\text { studies, } \mathrm{n} \\
\text { (total } \\
\text { studies) }\end{array}$ & Method of synthesis & Interventions & Outcomes & $\begin{array}{l}\text { Follow- } \\
\text { up }\end{array}$ & Summary of findings & $\begin{array}{l}\text { AMSTAR- } \\
2 \text { rating }\end{array}$ \\
\hline \multirow[t]{2}{*}{$\begin{array}{l}\text { Maon et al } \\
(2012)[63]\end{array}$} & \multirow[t]{2}{*}{$13(26)$} & \multirow[t]{2}{*}{$\begin{array}{l}\text { Meta-analysis and } \\
\text { narrative synthesis } \\
\text { of RCTs }\end{array}$} & \multirow[t]{2}{*}{ Internet } & \multirow[t]{2}{*}{$\begin{array}{l}\text { PA levels, sedentary } \\
\text { behavior, and MV- } \\
\text { PA }\end{array}$} & \multirow[t]{2}{*}{$\begin{array}{l}6 \\
\text { weeks-2 } \\
\text { years }\end{array}$} & $\begin{array}{l}54 \%(7 / 13) \text { of studies showed } \\
\text { statistically significant effects } \\
\text { on PA levels, such as increased } \\
\text { walking or decreased sedentary } \\
\text { behavior. However, a meta- } \\
\text { analysis on } 4 \text { studies with ex- } \\
\text { tractable data for the outcome } \\
\text { of moderate-to-vigorous week- } \\
\text { ly PA found a not statistically } \\
\text { significant improvement: } \\
\text { SMD } 0.15 \text { ( } 95 \% \text { CI } 20.06 \text { to } \\
0.35 ; P=.16)\end{array}$ & \multirow[t]{2}{*}{$\begin{array}{l}\text { Critically } \\
\text { low }\end{array}$} \\
\hline & & & & & & $\begin{array}{l}\text { Duration of studies and effects: } \\
10 \%(3 / 30) \text { of studies showed } \\
\text { positive effects when outcomes } \\
\text { were measured immediately } \\
\text { after the end of the interven- } \\
\text { tions. In total, } 37 \%(11 / 30) \text { of } \\
\text { studies that lasted } 3 \text { months or } \\
\text { less demonstrated positive out- } \\
\text { comes; } 43 \%(13 / 30) \text { of studies } \\
\text { with an intervention of } 3-6 \\
\text { months showed positive results; } \\
\text { and only } 10 \%(3 / 30) \text { interven- } \\
\text { tions that lasted longer than } 6 \\
\text { months were reported to have } \\
\text { positive results. }\end{array}$ & \\
\hline $\begin{array}{l}\text { Webb et al } \\
(2010)[113]\end{array}$ & $20(85)$ & $\begin{array}{l}\text { Meta-analysis of } \\
\text { RCTs }\end{array}$ & Internet & Level of PA & $\begin{array}{l}3-12 \\
\text { months }\end{array}$ & $\begin{array}{l}\text { Small effects on behavior were } \\
\text { observed for interventions that } \\
\text { targeted only PA (Cohen } \\
d+=0.24 ; \mathrm{k}=20 ; 95 \% \text { CI } 0.09 \text { to } \\
0.38 \text { ). }\end{array}$ & $\begin{array}{l}\text { Critically } \\
\text { low }\end{array}$ \\
\hline $\begin{array}{l}\text { Buchholz et } \\
\text { al (2013) } \\
\text { [27] (PA on- } \\
\text { ly) }\end{array}$ & $10(10)$ & $\begin{array}{l}\text { Narrative synthesis } \\
\text { of RCTs, quasiexper- } \\
\text { imental and, single } \\
\text { groups }\end{array}$ & $\begin{array}{l}\text { Mobile (SMS } \\
\text { text messag- } \\
\text { ing) }\end{array}$ & $\begin{array}{l}\text { Self-reported fre- } \\
\text { quency or pedome- } \\
\text { ter-reported steps } \\
\text { and level of PA }\end{array}$ & $\begin{array}{l}3-52 \\
\text { weeks }\end{array}$ & $\begin{array}{l}\text { Effect sizes across all studies } \\
\text { were positive; the median effect } \\
\text { size was } 0.5 \text { (medium) but het- } \\
\text { erogeneous. Sample sizes were } \\
\text { small. }\end{array}$ & $\begin{array}{l}\text { Critically } \\
\text { low }\end{array}$ \\
\hline $\begin{array}{l}\text { Elavsky } \\
\text { (2018) [31] } \\
\text { (PA only) }\end{array}$ & $50(52)$ & $\begin{array}{l}\text { Narrative synthesis } \\
\text { of RCTs and pre- } \\
\text { post studies }\end{array}$ & Mobile & $\begin{array}{l}\text { PA and sedentary } \\
\text { behavior }\end{array}$ & $\begin{array}{l}<3 \\
\text { months }\end{array}$ & $\begin{array}{l}59 \%(17 / 29) \text { of RCTs and } 62 \% \\
\text { (13/21) of pre-post studies sup- } \\
\text { ported the effectiveness of mo- } \\
\text { bile interventions to improve } \\
\text { PA, and } 9 \text { ( } 5 \text { of } 10 \text { RCTs and } \\
\text { all } 4 \text { pre-post) of } 14(64 \%) \\
\text { studies reduced sedentary be- } \\
\text { havior. }\end{array}$ & $\begin{array}{l}\text { Critically } \\
\text { low }\end{array}$ \\
\hline $\begin{array}{l}\text { Lyzwinksi et } \\
\text { al (2014) } \\
{[60]}\end{array}$ & $9(14)$ & $\begin{array}{l}\text { Meta-analysis of } \\
\text { RCTs }\end{array}$ & Mobile & Levels of PA & $\begin{array}{l}8 \\
\text { weeks- } \\
12 \\
\text { months }\end{array}$ & $\begin{array}{l}\text { Trials mostly found that PA } \\
\text { levels increased in the interven- } \\
\text { tion groups relative to the con- } \\
\text { trol groups. }\end{array}$ & $\begin{array}{l}\text { Critically } \\
\text { low }\end{array}$ \\
\hline $\begin{array}{l}\text { Maher et al } \\
(2014)[61]\end{array}$ & $4(10)$ & $\begin{array}{l}\text { Narrative synthesis } \\
\text { of studies with com- } \\
\text { parator group (con- } \\
\text { trol or within sub- } \\
\text { ject) }\end{array}$ & Mobile & Levels of PA & $\begin{array}{l}8 \\
\text { weeks- } \\
24 \\
\text { months }\end{array}$ & $\begin{array}{l}25 \%(1 / 4) \text { of studies demonstrat- } \\
\text { ed a significant change in PA } \\
\text { Cohen } d=0.84 \text { ( } 95 \% \text { CI }-0.49 \\
\text { to } 1.19) \text {. }\end{array}$ & $\begin{array}{l}\text { Critically } \\
\text { low }\end{array}$ \\
\hline $\begin{array}{l}\text { Muntaner et } \\
\text { al (2015) } \\
\text { [41] (PA on- } \\
\text { ly) }\end{array}$ & $11(11)$ & $\begin{array}{l}\text { Narrative synthesis } \\
\text { of all study types }\end{array}$ & Mobile & PA; exercise & $\begin{array}{l}2-24 \\
\text { weeks }\end{array}$ & $\begin{array}{l}55 \%(6 / 11) \text { of articles included } \\
\text { in this review reported signifi- } \\
\text { cant increases in PA levels. }\end{array}$ & $\begin{array}{l}\text { Critically } \\
\text { low }\end{array}$ \\
\hline
\end{tabular}




\begin{tabular}{|c|c|c|c|c|c|c|c|}
\hline Review & $\begin{array}{l}\text { Relevant } \\
\text { studies, } \mathrm{n} \\
\text { (total } \\
\text { studies) }\end{array}$ & Method of synthesis & Interventions & Outcomes & $\begin{array}{l}\text { Follow- } \\
\text { up }\end{array}$ & Summary of findings & $\begin{array}{l}\text { AMSTAR- } \\
2 \text { rating }\end{array}$ \\
\hline $\begin{array}{l}\text { O'Reilly et al } \\
\text { (2013) [42] } \\
\text { (PA only) }\end{array}$ & $12(22)$ & $\begin{array}{l}\text { Narrative synthesis } \\
\text { of RCTs }\end{array}$ & Mobile & $\begin{array}{l}\text { PA; sedentary behav- } \\
\text { ior; BMI; blood } \\
\text { lipids; blood pres- } \\
\text { sure; QoL }{ }^{\text {e }} \text {; adverse } \\
\text { effects }\end{array}$ & $\begin{array}{l}\text { Not re- } \\
\text { ported }\end{array}$ & $\begin{array}{l}75 \%(9 / 12) \text { of studies reported } \\
\text { significant changes in PA or } \\
\text { sedentary behavior. }\end{array}$ & $\begin{array}{l}\text { Critically } \\
\text { low }\end{array}$ \\
\hline $\begin{array}{l}\text { Palmer et al } \\
(2018)[111]\end{array}$ & $15(71)$ & $\begin{array}{l}\text { Meta-analysis of } \\
\text { RCTs }\end{array}$ & Mobile & Level of PA & $\begin{array}{l}3 \\
\text { months }\end{array}$ & $\begin{array}{l}\text { Trials of PA interventions re- } \\
\text { porting outcomes at } 3 \text { months } \\
\text { showed no benefits. }\end{array}$ & Moderate \\
\hline $\begin{array}{l}\text { Schoeppe et } \\
\text { al (2016) } \\
{[68]}\end{array}$ & $10(27)$ & $\begin{array}{l}\text { Narrative synthesis } \\
\text { of RCTs, random- } \\
\text { ized trials, con- } \\
\text { trolled trials, and } \\
\text { pre- and poststudies }\end{array}$ & Mobile & $\begin{array}{l}\text { PA; sedentary behav- } \\
\text { ior }\end{array}$ & $\begin{array}{l}1-24 \\
\text { weeks }\end{array}$ & $\begin{array}{l}59 \%(13 / 22) \text { of studies reported } \\
\text { significant improvements in } \\
\text { levels of PA; } 20 \%(1 / 5) \text { of } \\
\text { studies reported a significant } \\
\text { change in sedentary behavior. }\end{array}$ & $\begin{array}{l}\text { Critically } \\
\text { low }\end{array}$ \\
\hline $\begin{array}{l}\text { Elaheebocus } \\
\text { et al (2018) } \\
{[56]}\end{array}$ & $25(134)$ & $\begin{array}{l}\text { Narrative synthesis } \\
\text { of RCTs }\end{array}$ & Social media & Body weight & $\begin{array}{l}6-48 \\
\text { months }\end{array}$ & $\begin{array}{l}76 \%(19 / 25) \text { of studies using } \\
\text { online social networks had } \\
\text { positive results. }\end{array}$ & $\begin{array}{l}\text { Critically } \\
\text { low }\end{array}$ \\
\hline $\begin{array}{l}\text { Mita et al } \\
\text { (2016) [65] }\end{array}$ & $11(16)$ & $\begin{array}{l}\text { Meta-analysis of } \\
\text { RCTs }\end{array}$ & Social media & PA; weight change & $\begin{array}{l}1-12 \\
\text { months }\end{array}$ & $\begin{array}{l}\text { For PA, significant mean differ- } \\
\text { ence } 0.07 ; 95 \% \mathrm{CI}-0.25 \text { to } \\
0.38 ; \mathrm{k}=11 \text {. }\end{array}$ & Moderate \\
\hline $\begin{array}{l}\text { Williams et } \\
\text { al (2012) } \\
{[75]}\end{array}$ & $12(22)$ & $\begin{array}{l}\text { Meta-analysis of } \\
\text { RCTs }\end{array}$ & Social media & PA & $\begin{array}{l}3-24 \\
\text { months }\end{array}$ & $\begin{array}{l}\text { Meta-analysis showed no signif- } \\
\text { icant differences in changes in } \\
\text { PA (SMD } 0.13 ; 95 \% \text { CI }-0.04 \\
\text { to } 0.30 ; \mathrm{k}=12 \text { ). }\end{array}$ & $\begin{array}{l}\text { Critically } \\
\text { low }\end{array}$ \\
\hline $\begin{array}{l}\text { Willis et al } \\
\text { (2017) [76] }\end{array}$ & $3(5)$ & $\begin{array}{l}\text { Narrative synthesis } \\
\text { of all study types }\end{array}$ & Social media & Total PA & $\begin{array}{l}8 \\
\text { weeks-6 } \\
\text { months }\end{array}$ & $\begin{array}{l}\text { Only one study reported signif- } \\
\text { icant changes in levels of PA, } \\
\text { when the web-based social net- } \\
\text { work intervention included an } \\
\text { online support group. }\end{array}$ & $\begin{array}{l}\text { Critically } \\
\text { low }\end{array}$ \\
\hline $\begin{array}{l}\text { Johnson } \\
\text { (2017) [37] }\end{array}$ & $10(19)$ & $\begin{array}{l}\text { Narrative synthesis } \\
\text { of RCTs }\end{array}$ & Active gaming & $\begin{array}{l}\text { Behavioral and cog- } \\
\text { nitive outcomes }\end{array}$ & $\begin{array}{l}\text { Not re- } \\
\text { ported }\end{array}$ & $\begin{array}{l}\text { Findings were largely positive } \\
\text { for behavioral impacts, specifi- } \\
\text { cally the impact of gamification } \\
\text { for PA: } 80 \%(8 / 10) \text { positive and } \\
20 \%(2 / 10) \text { mixed. }\end{array}$ & $\begin{array}{l}\text { Critically } \\
\text { low }\end{array}$ \\
\hline $\begin{array}{l}\text { Peng et al } \\
\text { (2012) [43] } \\
\text { (PA only) }\end{array}$ & $4(12)$ & $\begin{array}{l}\text { Narrative synthesis } \\
\text { of all study designs }\end{array}$ & Active gaming & $\begin{array}{l}\text { Heart rate; energy } \\
\text { expenditure; and } \\
\text { oxygen uptake }\end{array}$ & $\begin{array}{l}\text { 6-12 } \\
\text { weeks }\end{array}$ & $\begin{array}{l}\text { Evidence does not support ac- } \\
\text { tive video games as an effective } \\
\text { tool to significantly increase } \\
\text { PA or exercise attendance. }\end{array}$ & $\begin{array}{l}\text { Critically } \\
\text { low }\end{array}$ \\
\hline
\end{tabular}




\begin{tabular}{|c|c|c|c|c|c|c|c|}
\hline Review & $\begin{array}{l}\text { Relevant } \\
\text { studies, } \mathrm{n} \\
\text { (total } \\
\text { studies) }\end{array}$ & Method of synthesis & Interventions & Outcomes & $\begin{array}{l}\text { Follow- } \\
\text { up }\end{array}$ & Summary of findings & $\begin{array}{l}\text { AMSTAR- } \\
2 \text { rating }\end{array}$ \\
\hline $\begin{array}{l}\text { Street et al } \\
(2017)[46] \\
\text { (PA only) }\end{array}$ & $9(9)$ & $\begin{array}{l}\text { Narrative synthesis } \\
\text { of studies with com- } \\
\text { parison or control } \\
\text { groups }\end{array}$ & Active gaming & $\begin{array}{l}\text { PA; maximum oxy- } \\
\text { gen uptake; power; } \\
\text { blood pressure; body } \\
\text { mass; body weight; } \\
\text { body fat; BMI; bal- } \\
\text { ance; speed; and } \\
\text { strength }\end{array}$ & $\begin{array}{l}6-12 \\
\text { weeks }\end{array}$ & $\begin{array}{l}\text { Moderate-to-high exergaming } \\
\text { participation was associated } \\
\text { with statistically significant } \\
\text { improvements in anthropomet- } \\
\text { ric outcomes but low participa- } \\
\text { tion was not associated with } \\
\text { anthropometric changes. } 38 \% \\
\text { (3/8) studies that investigated } \\
\text { anthropometric outcomes, in- } \\
\text { cluding BMI and body fat, } \\
\text { found a statistically significant } \\
\text { improvement, all } 3 \text { studies } \\
\text { showed positive health out- } \\
\text { comes associated with moder- } \\
\text { ate-to-high participation in ex- } \\
\text { ergaming; } 100 \% \text { ( } 3 / 3 \text { ) of stud- } \\
\text { ies that reported on PA frequen- } \\
\text { cy reported higher frequency in } \\
\text { the exergaming condition; } \\
\text { however, a different } 100 \% \text { ( } 3 / 3 \text { ) } \\
\text { of studies that reported on } \\
\text { overall PA found no statistical- } \\
\text { ly significant increases. }\end{array}$ & $\begin{array}{l}\text { Critically } \\
\text { low }\end{array}$ \\
\hline $\begin{array}{l}\text { Wieland et } \\
\text { al }(2012) \\
{[74]}\end{array}$ & $4(18)$ & $\begin{array}{l}\text { Meta-analysis of } \\
\text { RCTs, quasi-RCTs, } \\
\text { cluster RCTs, and } \\
\text { quasiexperimental } \\
\text { studies }\end{array}$ & $\begin{array}{l}\text { Computer-de- } \\
\text { livered }\end{array}$ & $\begin{array}{l}\text { Steps per day and } \\
\text { minutes walked con- } \\
\text { tinuously }\end{array}$ & $\begin{array}{l}4 \\
\text { weeks- } \\
30 \\
\text { months }\end{array}$ & $\begin{array}{l}\text { No studies demonstrated statis- } \\
\text { tically significant effects on } \\
\text { PA. }\end{array}$ & $\begin{array}{l}\text { Critically } \\
\text { low }\end{array}$ \\
\hline $\begin{array}{l}\text { Afshin et al } \\
(2016) \text { [99] }\end{array}$ & $33(224)$ & $\begin{array}{l}\text { Narrative synthesis } \\
\text { of RCTs and quasi- } \\
\text { experimental studies }\end{array}$ & $\begin{array}{l}\text { Various: inter- } \\
\text { net and mobile }\end{array}$ & Level of PA & $\begin{array}{l}1 \text { week- } \\
5 \text { years }\end{array}$ & $\begin{array}{l}88 \%(29 / 33) \text { of studies reported } \\
\text { significant improvement in PA; } \\
83 \%(5 / 6) \text { of phone interven- } \\
\text { tions were effective, including } \\
66 \%(2 / 3) \text { of SMS text messag- } \\
\text { ing interventions, } 100 \%(2 / 2) \\
\text { of apps, and } 100 \%(1 / 1) \text { of au- } \\
\text { tomated voice response. }\end{array}$ & $\begin{array}{l}\text { Critically } \\
\text { low }\end{array}$ \\
\hline $\begin{array}{l}\text { Carvalho de } \\
\text { Menzes et al } \\
(2016)[53]\end{array}$ & $13(18)$ & $\begin{array}{l}\text { Narrative synthesis } \\
\text { of all study designs }\end{array}$ & $\begin{array}{l}\text { Various: } \\
\text { email, tele- } \\
\text { phone, web- } \\
\text { sites }\end{array}$ & Level of PA & $\begin{array}{l}1-36 \\
\text { months }\end{array}$ & $\begin{array}{l}\text { Most studies demonstrated sta- } \\
\text { tistically significant improve- } \\
\text { ments in the level of PA. }\end{array}$ & $\begin{array}{l}\text { Critically } \\
\text { low }\end{array}$ \\
\hline $\begin{array}{l}\text { Hakala et al } \\
\text { (2017) [34] } \\
\text { (PA only) }\end{array}$ & $13(23)$ & $\begin{array}{l}\text { Meta-analysis of } \\
\text { RCTs }\end{array}$ & $\begin{array}{l}\text { Various: mo- } \\
\text { bile, text mes- } \\
\text { sages, pedome- } \\
\text { ters, wear- } \\
\text { ables, email }\end{array}$ & $\begin{array}{l}\text { PA: self-reported or } \\
\text { using an accelerome- } \\
\text { ter or pedometer }\end{array}$ & $\begin{array}{l}3 \\
\text { weeks- } \\
24 \\
\text { months }\end{array}$ & $\begin{array}{l}\text { No differences were observed } \\
\text { between the experimental and } \\
\text { control groups (risk ratio } 1.03 \text {; } \\
95 \% \text { CI } 0.92 \text { to } 1.15 ; P=.57 \text { ). }\end{array}$ & $\begin{array}{l}\text { Critically } \\
\text { low }\end{array}$ \\
\hline $\begin{array}{l}\text { Muellmann } \\
\text { et al (2018) } \\
\text { [39] (PA on- } \\
\text { ly) }\end{array}$ & $13(20)$ & $\begin{array}{l}\text { Narrative synthesis } \\
\text { of experimental de- } \\
\text { signs and quasiexper- } \\
\text { imental studies }\end{array}$ & $\begin{array}{l}\text { SMS text mes- } \\
\text { saging and in- } \\
\text { ternet }\end{array}$ & $\begin{array}{l}\text { PA and number of } \\
\text { steps per day }\end{array}$ & $\begin{array}{l}4 \\
\text { weeks- } \\
24 \\
\text { months }\end{array}$ & $\begin{array}{l}75 \%(3 / 4) \text { of studies using mo- } \\
\text { bile phones demonstrated signif- } \\
\text { icant differences in the level of } \\
\text { PA or steps per day (mixed } \\
\text { controls). In } 100 \%(9 / 9) \text { of } \\
\text { studies, internet interventions } \\
\text { significantly increased PA } \\
\text { compared with nonactive con- } \\
\text { trols. }\end{array}$ & $\begin{array}{l}\text { Critically } \\
\text { low }\end{array}$ \\
\hline $\begin{array}{l}\text { Muller and } \\
\text { Khoo (2014) } \\
\text { [40] (PA on- } \\
\text { ly) }\end{array}$ & $4(16)$ & $\begin{array}{l}\text { Narrative synthesis } \\
\text { of RCTs and quasiex- } \\
\text { perimental studies }\end{array}$ & $\begin{array}{l}\text { Various: inter- } \\
\text { net and mobile }\end{array}$ & PA & $\begin{array}{l}1 \text { week- } \\
18 \\
\text { months }\end{array}$ & $\begin{array}{l}75 \%(3 / 4) \text { of studies reported } \\
\text { significant improvements in } \\
\text { PA; } 25 \%(1 / 4) \text { of studies report- } \\
\text { ed nonsignificant decrease in } \\
\text { PA. }\end{array}$ & $\begin{array}{l}\text { Critically } \\
\text { low }\end{array}$ \\
\hline
\end{tabular}




\begin{tabular}{|c|c|c|c|c|c|c|c|}
\hline Review & $\begin{array}{l}\text { Relevant } \\
\text { studies, n } \\
\text { (total } \\
\text { studies) }\end{array}$ & Method of synthesis & Interventions & Outcomes & $\begin{array}{l}\text { Follow- } \\
\text { up }\end{array}$ & Summary of findings & $\begin{array}{l}\text { AMSTAR- } \\
2 \text { rating }\end{array}$ \\
\hline $\begin{array}{l}\text { Stephenson } \\
\text { et al (2017) } \\
\text { [45] (PA on- } \\
\text { ly) }\end{array}$ & $15(17)$ & $\begin{array}{l}\text { Meta-analysis of } \\
\text { RCTs }\end{array}$ & $\begin{array}{l}\text { Various: mo- } \\
\text { bile messag- } \\
\text { ing, mobile } \\
\text { apps, website, } \\
\text { wearable tech- } \\
\text { nology }\end{array}$ & Sedentary behavior & $\begin{array}{l}5 \text { days- } \\
24 \\
\text { months }\end{array}$ & $\begin{array}{l}\text { Interventions using computer } \\
\text { and mobile and wearable tech- } \\
\text { nologies can be effective in re- } \\
\text { ducing sedentary behavior. Ef- } \\
\text { fectiveness appeared most } \\
\text { prominent in the short-term and } \\
\text { lessened over time. Meta-analy- } \\
\text { sis of } 88 \% \text { (15/17) of RCTs } \\
\text { suggested that computer, mo- } \\
\text { bile, and wearable technology } \\
\text { tools resulted in a mean reduc- } \\
\text { tion of }-41.28 \text { min/day of sit- } \\
\text { ting time ( } 95 \% \text { CI }-60.99 \text { to } \\
\left.-21.58 ; I^{2}=77 \%\right) \text {. The pooled } \\
\text { effects showed mean reductions } \\
\text { at short ( } \leq 3 \text { months), medium } \\
\text { (>3 to } 6 \text { months), and long-term } \\
\text { (>6 months) follow-up of } \\
-42.42 \text { min/day, }-37.23 \\
\text { min/day, and }-1.65 \text { min/day, } \\
\text { respectively. }\end{array}$ & $\begin{array}{l}\text { Critically } \\
\text { low }\end{array}$ \\
\hline
\end{tabular}

\section{Nonactive controls}

Jenkins et al $5(22)$

(2009) [36]

(PA only)
Narrative synthesis Internet of RCTs
PA

$0-24$ months

Results were mixed; internet

Critically interventions can be effective, low compared with control conditions, although poor compliance was an issue. $50 \%(2 / 4)$ studies reported an increase in PA compared with nonactive controls while 2 studies found no difference.

\begin{tabular}{|c|c|c|}
\hline $\begin{array}{l}\text { Bock et al } \\
(2014)[24] \\
\text { (PA only) }\end{array}$ & $4(50)$ & $\begin{array}{l}\text { Narrative synthesis } \\
\text { of RCTs and quasiex- } \\
\text { perimental studies }\end{array}$ \\
\hline $\begin{array}{l}\text { Krebs et al } \\
(2010) \text { [109] }\end{array}$ & $25(76)$ & $\begin{array}{l}\text { Meta-analysis of } \\
\text { RCTs }\end{array}$ \\
\hline $\begin{array}{l}\text { Bort-Roig et } \\
\text { al (2014) } \\
{[25] \text { (PA on- }}\end{array}$ & $5(26)$ & $\begin{array}{l}\text { Narrative synthesis } \\
\text { of comparative and } \\
\text { pre-postdesign }\end{array}$ \\
\hline
\end{tabular}

Internet or computer

Weekly PA, proportion of sufficiently active persons; step counts

\section{Computer-de- Minutes of PA} livered

Mobile

PA (steps); energy expenditure; body weight and body fat; blood pressure and cholesterol; QoL

\section{.}

Interventions had a nonsignifi- Critically weeks-3 cant, positive effect on PA years $\quad(P=.88)$.

1-18 The mean effect size was months $g=0.16(95 \%$ CI 0.10 to 0.21$)$.

$280 \%(4 / 5)$ of studies assessing months PA increases, with mean PA increases ranging from 800 to 1104 steps/day. Studies were small with differences in baseline characteristics.

PA, MVPA, walking 1-52 and sedentary behav- weeks ior

Not effective for MVPA outcomes, based only on adult studies SMD 0.14 (95\% CI -0.10 to 0.37 ). For sedentary behavior outcomes, SMD -0.21 (95\% CI -0.59 to 0.18 ).

Freak-Poli et 4 (4)

Narrative synthesis Wearable al (2013) [32] (PA onof RCTs and cluster technology 1y)

PA; sedentary behav- 3-8 ior; BMI; blood months lipids; blood pressure; QoL; adverse effects
Overall, there was insufficient evidence to assess the effectiveness of pedometer interventions in the workplace. $75 \%(3 / 4)$ of studies compared with a minimal control group, 33\% (1/3) of studies observed an increase in PA under a pedometer program, but the other two did not find a significant difference. 


\begin{tabular}{|c|c|c|c|c|c|c|c|}
\hline Review & $\begin{array}{l}\text { Relevant } \\
\text { studies, } \mathrm{n} \\
\text { (total } \\
\text { studies) }\end{array}$ & Method of synthesis & Interventions & Outcomes & $\begin{array}{l}\text { Follow- } \\
\text { up }\end{array}$ & Summary of findings & $\begin{array}{l}\text { AMSTAR- } \\
2 \text { rating }\end{array}$ \\
\hline $\begin{array}{l}\text { An et al } \\
(2017)[49]\end{array}$ & $21(22)$ & $\begin{array}{l}\text { Meta-analysis of } \\
\text { RCTs, pre-post stud- } \\
\text { ies, and cohort stud- } \\
\text { ies }\end{array}$ & Social media & $\begin{array}{l}\text { PA, sedentary behav- } \\
\text { ior }\end{array}$ & $\begin{array}{l}3-102 \\
\text { weeks }\end{array}$ & $\begin{array}{l}\text { Interventions increased daily } \\
\text { number of steps taken by } 1530 \\
\text { ( } 95 \% \text { CI } 82 \text { to } 2979 \text { ). However, } \\
\text { they were not associated with } \\
\text { energy expenditure, total PA, } \\
\text { or MVPA. }\end{array}$ & $\begin{array}{l}\text { Critically } \\
\text { low }\end{array}$ \\
\hline $\begin{array}{l}\text { Tsoli et al } \\
(2018) \text { [73] }\end{array}$ & $3(15)$ & $\begin{array}{l}\text { Meta-analysis of } \\
\text { RCTs }\end{array}$ & $\begin{array}{l}\text { Interactive } \\
\text { voice respons- } \\
\text { es }\end{array}$ & PA & $\begin{array}{l}6 \\
\text { weeks- } \\
12 \\
\text { months }\end{array}$ & $\begin{array}{l}\text { Interventions led to a small but } \\
\text { statistically significant increase } \\
\text { in PA ( } g=0.254 ; 95 \% \text { CI } 0.068 \\
\text { to } 0.439 ; \mathrm{k}=3 ; P=.007) \text {. }\end{array}$ & $\begin{array}{l}\text { Critically } \\
\text { low }\end{array}$ \\
\hline \multicolumn{8}{|l|}{ Active controls } \\
\hline $\begin{array}{l}\text { Beishuizen } \\
\text { et al (2016) } \\
{[102]}\end{array}$ & $5(57)$ & $\begin{array}{l}\text { Meta-analysis of } \\
\text { RCTs }\end{array}$ & Internet & Level of PA & $\begin{array}{l}4 \\
\text { weeks-3 } \\
\text { months }\end{array}$ & $\begin{array}{l}\text { Interventions led to an increase } \\
\text { in PA (SMD } 0.25 ; 95 \% \text { CI } 0.10 \\
\text { to } 0.39 \text { ). }\end{array}$ & Low \\
\hline $\begin{array}{l}\text { Covolo et al } \\
(2017)[104]\end{array}$ & $23(40)$ & $\begin{array}{l}\text { Narrative synthesis } \\
\text { of RCTs }\end{array}$ & Mobile apps & $\begin{array}{l}\text { Daily steps, frequen- } \\
\text { cy, and intensity of } \\
\text { PA }\end{array}$ & $\begin{array}{l}6 \\
\text { months- } \\
2 \text { years }\end{array}$ & $\begin{array}{l}30 \%(7 / 23) \text { of RCTs showed a } \\
\text { significant increase in PA in the } \\
\text { intervention group (measured } \\
\text { in daily steps, frequency of PA, } \\
\text { or level of intensity), } 48 \% \\
\text { (11/23) of studies did not show } \\
\text { a significant increase, and in } \\
21 \%(5 / 23) \text { studies, outcome } \\
\text { measures were inconsistent in } \\
\text { whether there was a significant } \\
\text { difference between intervention } \\
\text { and control. }\end{array}$ & $\begin{array}{l}\text { Critically } \\
\text { low }\end{array}$ \\
\hline $\begin{array}{l}\text { Mateo et al } \\
(2015)[38] \\
\text { (PA only) }\end{array}$ & $10(11)$ & $\begin{array}{l}\text { Meta-analysis of } \\
\text { controlled trials }\end{array}$ & Mobile apps & $\begin{array}{l}\text { PA, MVPA, and } \\
\text { steps }\end{array}$ & $\begin{array}{l}6 \\
\text { weeks-9 } \\
\text { months }\end{array}$ & $\begin{array}{l}\text { Compared with the control } \\
\text { group, use of a mobile phone } \\
\text { app was associated with signif- } \\
\text { icant changes in body weight } \\
\text { and BMI of }-1.04 \mathrm{~kg}(95 \% \mathrm{CI} \\
\left.-1.75 \text { to }-0.34 ; \mathrm{I}^{2}=41 \%\right) \text { and } \\
-0.43 \mathrm{~kg} / \mathrm{m}^{2}(95 \% \mathrm{CI}-0.74 \text { to } \\
\left.-0.13 ; \mathrm{I}^{2}=50 \%\right) \text {, respectively } \\
(\mathrm{k}=9) ; \text { however, a nonsignifi- } \\
\text { cant difference in PA was ob- } \\
\text { served between the intervention } \\
\text { and comparison groups (SMD } \\
0.40 ; 95 \% \text { CI }-0.07 \text { to } 0.87 \text {; } \\
\left.\mathrm{I}^{2}=93 \%\right) \text {. }\end{array}$ & $\begin{array}{l}\text { Critically } \\
\text { low }\end{array}$ \\
\hline $\begin{array}{l}\text { Song et al } \\
\text { (2018) }[44] \\
\text { (PA only) }\end{array}$ & $6(8)$ & $\begin{array}{l}\text { Narrative synthesis } \\
\text { of all study types }\end{array}$ & Mobile & $\begin{array}{l}\text { PA (frequency and } \\
\text { step count); BMI; } \\
\text { blood glucose }\end{array}$ & $\begin{array}{l}4 \\
\text { weeks-6 } \\
\text { months }\end{array}$ & $\begin{array}{l}\text { Significant effects on frequency } \\
\text { of PA in } 80 \%(4 / 5) \text { of studies } \\
\text { (though the effect was reported } \\
\text { to have disappeared after the } \\
12 \text {-week follow-up), step count } \\
\text { in } 66 \%(2 / 3) \text { of studies, BMI in } \\
50 \%(2 / 4) \text { of studies, and reduc- } \\
\text { tion in glucose in } 100 \%(2 / 2) \\
\text { studies. }\end{array}$ & $\begin{array}{l}\text { Critically } \\
\text { low }\end{array}$ \\
\hline
\end{tabular}




\begin{tabular}{|c|c|c|c|c|c|c|c|}
\hline Review & $\begin{array}{l}\text { Relevant } \\
\text { studies, } \mathrm{n} \\
\text { (total } \\
\text { studies) }\end{array}$ & Method of synthesis & Interventions & Outcomes & $\begin{array}{l}\text { Follow- } \\
\text { up }\end{array}$ & Summary of findings & $\begin{array}{l}\text { AMSTAR- } \\
2 \text { rating }\end{array}$ \\
\hline $\begin{array}{l}\text { Cheatham et } \\
\text { al (2018) } \\
\text { [28] (PA on- } \\
\text { ly) }\end{array}$ & $25(25)$ & $\begin{array}{l}\text { Narrative synthesis } \\
\text { of controlled clinical } \\
\text { trials }\end{array}$ & $\begin{array}{l}\text { Wearable } \\
\text { technology }\end{array}$ & $\begin{array}{l}\text { PA; BMI; weight; } \\
\text { blood pressure; } \\
\text { Resting Energy Ex- } \\
\text { penditure; body } \\
\text { composition; cardio- } \\
\text { vascular fitness; } \\
\text { work productivity } \\
\text { and absenteeism; } \\
\text { waist circumference; } \\
\text { blood parameters }\end{array}$ & $\begin{array}{l}3 \\
\text { weeks- } \\
24 \\
\text { months }\end{array}$ & $\begin{array}{l}\text { An activity tracker combined } \\
\text { with a comprehensive weight } \\
\text { loss program may provide supe- } \\
\text { rior short-term ( } \leq 6 \text { months) re- } \\
\text { sults than a standard weight } \\
\text { loss program in middle aged or } \\
\text { older adults. } 80 \%(20 / 25) \text { of } \\
\text { studies reported higher weight } \\
\text { loss when an activity tracker } \\
\text { was used with a weight loss in- } \\
\text { tervention. }\end{array}$ & $\begin{array}{l}\text { Critically } \\
\text { low }\end{array}$ \\
\hline
\end{tabular}

aA: physical activity.

${ }^{b}$ MVPA: moderate-to-vigorous physical activity.

${ }^{\mathrm{c}} \mathrm{RCT}$ : randomized controlled trial.

${ }^{\mathrm{d}} \mathrm{SMD}$ : standardized mean difference.

${ }^{\mathrm{e}} \mathrm{QoL}$ : quality of life.

Evidence for the effectiveness of internet interventions on physical activity has been mixed. In total, 5 out of 10 reviews were positive [26,29,33,108,113], including 2 meta-analyses that found small but significant effects of internet interventions: Cohen $d=0.14$ [29] and Cohen $d+=0.24$ [113]. However, there was significant heterogeneity across studies [29]. In contrast, 5 studies were not positive $[35,47,63,100,110]$, one of which was very unfavorable, with only 1 of $9(11 \%)$ studies demonstrating an effect of the intervention [100]. Two meta-analyses found that the effect of internet interventions on physical activity was not significant $[63,110]$.

A total of 4 reviews of social media interventions were mixed. In total, 2 meta-analyses of social media interventions found no significant difference in changes in physical activity [65,75], and a narrative synthesis reported mixed results [76]. However, one narrative synthesis found that $76 \%$ of studies using web-based social networks had positive results for physical activity [56].

The results of the mobile interventions to improve health were more positive. In total, 8 of $10(80 \%)$ narrative syntheses reported a majority of positive results [25,27,31,39,41,42,60,99], with one reporting an increase of 800 to 1104 steps per day [25]. One review noted that effective interventions used SMS text messaging communication or self-monitoring [42]. A review that was specifically on SMS text messaging reported that effect sizes were all greater than 0.20 , and the median was 0.50 , a medium effect size [27]. In contrast, 2 narrative syntheses reported that most mobile trials did not show any benefits $[61,111]$.

There was mixed evidence of active gaming across 3 reviews. One review found that gamification has a positive impact on physical activity and found evidence that gamification can increase motivation to exercise [37]. However, another review found a positive effect on attendance but not on physical activity or BMI [46]. A third review found that active gaming did not support increases in either physical activity or attendance [43].

Computer-delivered interventions in physical activity behaviors did not have consistent results in either weight loss or weight maintenance trials [74].

In total, 4 of the 5 reviews assessing a variety of interventions found favorable results $[40,45,53,99]$. One of the narrative syntheses reported a wide range of values for improvement, from 1.5 to 153 extra minutes of physical activity a week and 1000 to 2600 steps per day [99]. A meta-analysis found that computer, mobile, and wearable technology led to a mean change of -41.28 minutes per day of sitting time (a reduction in sitting time) [45]. However, one meta-analysis found no difference between the experimental and control groups [34].

\section{Effectiveness of Digital Interventions on Physical Activity Compared With Nonactive Controls}

Compared with minimal controls, evidence for the effectiveness of digital interventions has been mixed.

For internet interventions, one review found favorable evidence [39], another found unfavorable evidence [24], and a third found mixed evidence [36].

The 3 reviews of mobile interventions have also provided mixed evidence. One narrative synthesis found that interventions were effective, with 4 studies ( 3 pre-post and 1 comparative) reporting increases of 800 to 1104 steps per day [25]. However, another study found that mobile interventions were not effective in increasing physical activity of moderate-to-vigorous intensity or in decreasing sedentary behavior [30]. Wearables were also not very effective, with only 1 of 3 (33\%) studies comparing a pedometer with a minimal control showing increased physical activity [32].

Social media-based interventions increased the daily number of steps taken by 1530 steps per day [49]. However, they were not associated with energy expenditure, total physical activity, or moderate-to-vigorous physical activity.

There were small effect sizes for both computer-delivered interventions $(g=0.16) \quad$ [109] and interactive voice response-based interventions $(g=0.254)$ [73]. 


\section{Effectiveness of Digital Interventions on Physical Activity Compared With Active Controls}

There were mixed results compared with active controls.

A meta-analysis of internet interventions found an increase in physical activity with an SMD of 0.25 compared with active controls [102].

In total, 3 reviews of mobile phones had active controls, and there were mixed results. A meta-analysis found that the use of a mobile phone app was associated with significant changes in body weight $(-1.04 \mathrm{~kg})$ and BMI $\left(-0.43 \mathrm{~kg} / \mathrm{m}^{2}\right)$; however, there was no significant difference in physical activity between the 2 groups [38]. A narrative synthesis app was also not favorable for assessing changes in physical activity, with less than half of the studies showing a significant increase in physical activity in the intervention group [104]. However, another narrative synthesis of general mobile interventions found that most studies had interventions that led to changes in body weight, increases in step count, and increases in frequency of physical activity [44].

There were also promising results from a review on wearables: when an activity tracker is combined with a comprehensive weight loss program, it may provide superior short-term $(\leq 6$ months) results than a standard weight loss program in middle-aged or older adults (>30 years) [28].

\section{Sustainability of Effects on Physical Activity at Follow-Up}

There was little evidence on sustainability, as many physical activity studies did not have follow-up assessment postintervention or only had follow-ups relatively soon after the intervention end point.
There is some evidence that digital interventions can have sustained effects. A meta-analysis assessing combinations of digital technologies found that the pooled effects showed mean changes (reductions) at short ( $\leq 3$ months), medium (3 to 6 months), and long-term follow-up (>6 months) of -42.42 minutes per day, -37.23 minutes per day, and -1.65 minutes per day, respectively [45]. A meta-analysis of internet interventions also found a small but significant effect on physical activity for follow-ups at least six months postintervention (Cohen $d=0.11$ ) [29]. The sustainability of internet interventions was also supported by a narrative synthesis that found that only 12 of $35(34 \%)$ studies had follow-up assessments, which ranged from 7 weeks to 15 months postprogram; 10 out of 12 (83\%) studies demonstrated successful maintenance of physical activity and/or secondary measures indicative of positive changes in physical activity; however, follow-up durations were primarily shorter: in 9 studies, follow-up was conducted at less than 12 months [26]. However, for mobile interventions, 2 reviews found evidence that effects tended to decrease in the long term [31], with effects disappearing after as little as 12 weeks [44].

\section{Diet and Physical Activity (Weight Management)}

\section{Review Characteristics}

A total of 35 reviews reported on both diet and physical activity. The breakdown of their characteristics is shown in Multimedia Appendix 6.

\section{Effectiveness of Digital Interventions on Diet and Physical Activity (Weight Loss) Compared With Mixed (Active and Nonactive) Controls}

Overall, digital interventions were generally found to be effective, with mobile phone interventions in particular having consistently positive results (Table 5). 
Table 5. Results of reviews on diet and physical activity combined, ordered by type of control and further ordered by mode of delivery of intervention.

\begin{tabular}{|c|c|c|c|c|c|c|c|}
\hline Review & $\begin{array}{l}\text { Relevant } \\
\text { studies, n } \\
\text { (total } \\
\text { studies) }\end{array}$ & $\begin{array}{l}\text { Method of synthe- } \\
\text { sis }\end{array}$ & Interventions & Outcomes & $\begin{array}{l}\text { Follow- } \\
\text { up }\end{array}$ & Summary of findings & $\begin{array}{l}\text { AM- } \\
\text { STAR-2 } \\
\text { rating }\end{array}$ \\
\hline
\end{tabular}

\section{Mixed (active and nonactive) controls}

Aalbers et al $5(10)$

(2011) [47]

20 (29)

(2014) [100]

Narrative synthesis Internet

of RCTs ${ }^{\mathrm{a}}$ and non-

randomized pre-

post controlled tri-

als

Narrative synthesis Internet of RCTs
Beishuizen [102]
7 (57) Meta-analysis of Internet RCTs

Narrative synthesis Internet of all study types

Fry et al (2009) [57]

8 (19)

Hou et al 7 (38) (2013) [108]

Manzoni et [62]
Narrative synthesis Internet of all study types

\section{Body weight and 1.5-6.5 body weight regain months}

\section{Weight, BMI,} waist circumference, and body fat

Systolic blood pressure, diastolic months blood pressure, $\mathrm{HbA}_{1 \mathrm{c}} \mathrm{b}$ level, cholesterol level, weight, and level of physical activity
Body fat, weight, 0-12 and dietary fat in- months take

Weight loss and 3-24 weight loss mainte- months nance
$40 \%(2 / 5)$ of studies reported ef- Critically fect sizes on body weight with low small-to-medium significant effects; 1 study reported weight regain but did not reach significance.

Modest improvements were observed in more than half of the studies with weight-related outcomes; 20 studies reported on body weight: $75 \%(15 / 20)$ of high quality and 5 of $20(25 \%)$ low quality); 47\% (7/15) highquality studies reported significant improvement.

There was a significant reduction Low in systolic blood pressure $\left(\mathrm{MD}^{\mathrm{C}}\right.$ $-2.66 \mathrm{~mm} \mathrm{Hg} ; 95 \% \mathrm{CI}-3.81$ to $-1.52)$, diastolic blood pressure (MD -1.26 mm Hg; 95\% CI -1.92 to -0.60$), \mathrm{HbA}_{1 \mathrm{c}}$ level (MD $-0.13 \%$; $95 \% \mathrm{CI}-0.22$ to $-0.05), \mathrm{LDL}^{\mathrm{d}}$ cholesterol level (MD-2.18 mg/dL; 95\% CI -3.96 to -0.41$)$, weight (MD $-1.34 \mathrm{~kg}$; $95 \% \mathrm{CI}-1.91$ to -0.77 ), and an increase in physical activity $\left(\mathrm{SMD}^{\mathrm{e}} 0.25 ; 95 \% \mathrm{CI} 0.10\right.$ to $0.39)$.

Diet and physical 8 weeksactivity 30 months

There were generally positive enough evidence to know whether the medium in which prompts were sent through affected their effectiveness but personal contact with a counsellor did enhance effectiveness.

In $71 \%(5 / 7)$ of studies, interven- Critically tion groups lost more body fat, low body weight, and dietary fat intake and maintained higher weight loss at 12 months.

Internet-based weight loss inter- Critically ventions enhanced by profession- low al feedback provided through the internet are more effective for weight loss than website-only programs but less effective than telephone counselling. 93\% $(13 / 14)$ of studies showed a further improvement in mean weight loss (weight maintenance) after the end of the trials. 


\begin{tabular}{|c|c|c|c|c|c|c|c|}
\hline Review & $\begin{array}{l}\text { Relevant } \\
\text { studies, } \mathrm{n} \\
\text { (total } \\
\text { studies) }\end{array}$ & $\begin{array}{l}\text { Method of synthe- } \\
\text { sis }\end{array}$ & Interventions & Outcomes & $\begin{array}{l}\text { Follow- } \\
\text { up }\end{array}$ & Summary of findings & $\begin{array}{l}\text { AM- } \\
\text { STAR-2 } \\
\text { rating }\end{array}$ \\
\hline $\begin{array}{l}\text { Seo et al } \\
(2015)[69]\end{array}$ & $31(31)$ & $\begin{array}{l}\text { Meta-analysis of } \\
\text { RCTs }\end{array}$ & Internet & $\begin{array}{l}\text { Waist circumfer- } \\
\text { ence }\end{array}$ & $\begin{array}{l}4 \text { weeks- } \\
2 \text { years }\end{array}$ & $\begin{array}{l}\text { Internet-based interventions } \\
\text { showed a significant reduction } \\
\text { in waist circumference (mean } \\
\text { change }-2.99 \mathrm{~cm} ; 95 \% \mathrm{CI}-3.68 \\
\text { to }-2.30 ; \mathrm{I}^{2}=93.3 \% \text { ) and signifi- } \\
\text { cantly better effects on waist cir- } \\
\text { cumference loss (mean loss } 2.38 \\
\text { cm; } 95 \% \text { CI } 1.61 \text { to } 3.25 \text {; } \\
\mathrm{I}^{2}=97.2 \% \text { ) than minimal interven- } \\
\text { tions such as information-only } \\
\text { groups; no differences with re- } \\
\text { spect to waist circumference } \\
\text { change between internet-based } \\
\text { interventions and paper-, phone- } \\
\text {, or person-based interventions } \\
\text { (mean change }-0.61 \mathrm{~cm} ; 95 \% \mathrm{CI} \\
-2.05 \text { to } 0.83 ; P=.42 ; \mathrm{k}=31 \text { ). }\end{array}$ & $\begin{array}{l}\text { Critically } \\
\text { low }\end{array}$ \\
\hline $\begin{array}{l}\text { Sherrington } \\
\text { et al }(2016) \\
{[70]}\end{array}$ & $12(12)$ & $\begin{array}{l}\text { Meta-analysis of } \\
\text { RCTs }\end{array}$ & Internet & Weight loss & $\begin{array}{l}3-24 \\
\text { months }\end{array}$ & $\begin{array}{l}\text { The internet-delivered weight } \\
\text { loss interventions providing per- } \\
\text { sonalized feedback resulted in an } \\
\text { MD of } 2.13 \mathrm{~kg}(P<.001) \text { greater } \\
\text { weight loss in comparison with } \\
\text { control groups receiving no per- } \\
\text { sonalized feedback. Heterogene- } \\
\text { ity levels showed considerable } \\
\text { and significant heterogeneity } \\
\left(\mathrm{I}^{2}=99 \% \text {; } P<.001\right) \text { between con- } \\
\text { trol groups not receiving person- } \\
\text { alized feedback and the internet- } \\
\text { delivered weight loss interven- } \\
\text { tions providing personalized } \\
\text { feedback. }\end{array}$ & $\begin{array}{l}\text { Critically } \\
\text { low }\end{array}$ \\
\hline $\begin{array}{l}\text { Elaheebocus } \\
\text { et al (2018) } \\
{[56]}\end{array}$ & $11(134)$ & $\begin{array}{l}\text { Narrative synthesis } \\
\text { of RCTs }\end{array}$ & Social media & Body weight & $\begin{array}{l}6-48 \\
\text { months }\end{array}$ & $\begin{array}{l}82 \%(9 / 11) \text { of studies using web- } \\
\text { based social networks had posi- } \\
\text { tive results for weight loss. }\end{array}$ & $\begin{array}{l}\text { Critically } \\
\text { low }\end{array}$ \\
\hline $\begin{array}{l}\text { Maher et al } \\
(2014)[61]\end{array}$ & $5(10)$ & Narrative synthesis & Social media & Weight & $\begin{array}{l}8 \text { weeks- } \\
24 \\
\text { months }\end{array}$ & $\begin{array}{l}\text { Findings were mixed, from negli- } \\
\text { gible to large effect sizes for } \\
\text { weight loss. }\end{array}$ & $\begin{array}{l}\text { Critically } \\
\text { low }\end{array}$ \\
\hline $\begin{array}{l}\text { Mita et al } \\
(2016)[65]\end{array}$ & $10(16)$ & $\begin{array}{l}\text { Meta-analysis of } \\
\text { RCTs }\end{array}$ & Social media & Weight change & $\begin{array}{l}1-12 \\
\text { months }\end{array}$ & $\begin{array}{l}\text { Meta-analysis of all trials showed } \\
\text { no significant differences for } \\
\text { body weight (significant mean } \\
\text { difference } 0.07 ; 95 \% \mathrm{CI}-0.17 \\
\text { to } 0.20 \text { ). }\end{array}$ & Moderate \\
\hline $\begin{array}{l}\text { Williams et } \\
\text { al }(2012) \\
{[75]}\end{array}$ & $10(22)$ & $\begin{array}{l}\text { Meta-analysis of } \\
\text { RCTs }\end{array}$ & Social media & $\begin{array}{l}\text { BMI; body weight; } \\
\text { diet }\end{array}$ & $\begin{array}{l}3 \text { months- } \\
24 \\
\text { months }\end{array}$ & $\begin{array}{l}\text { Meta-analysis showed no signifi- } \\
\text { cant differences in changes in } \\
\text { weight (SMD } 0 ; 95 \% \text { CI }-0.19 \\
\text { to } 0.19 ; 10 \text { studies); however, } \\
\text { pooled results from } 5 \text { studies } \\
\text { showed a significant decrease in } \\
\text { dietary fat consumption with so- } \\
\text { cial media (SMD }-0.35 ; 95 \% \text { CI } \\
-0.68 \text { to }-0.02) \text {. }\end{array}$ & $\begin{array}{l}\text { Critically } \\
\text { low }\end{array}$ \\
\hline
\end{tabular}




\begin{tabular}{|c|c|c|c|c|c|c|c|}
\hline Review & $\begin{array}{l}\text { Relevant } \\
\text { studies, } \mathrm{n} \\
\text { (total } \\
\text { studies) }\end{array}$ & $\begin{array}{l}\text { Method of synthe- } \\
\text { sis }\end{array}$ & Interventions & Outcomes & $\begin{array}{l}\text { Follow- } \\
\text { up }\end{array}$ & Summary of findings & $\begin{array}{l}\text { AM- } \\
\text { STAR-2 } \\
\text { rating }\end{array}$ \\
\hline $\begin{array}{l}\text { Willis et al } \\
(2017)[76]\end{array}$ & $5(5)$ & $\begin{array}{l}\text { Narrative synthesis } \\
\text { of all study types }\end{array}$ & Social media & $\begin{array}{l}\text { Body weight; body } \\
\text { composition; blood } \\
\text { pressure; and blood } \\
\text { markers }\end{array}$ & $\begin{array}{l}8 \text { weeks- } \\
6 \text { months }\end{array}$ & $\begin{array}{l}100 \%(5 / 5) \text { of studies reported a } \\
\text { reduction in baseline weight. } \\
60 \%(3 / 5) \text { of studies reported } \\
\text { significant decreases in body } \\
\text { weight when online social net- } \\
\text { works was paired with health } \\
\text { educator support. Only one study } \\
\text { reported a clinically significant } \\
\text { weight loss of } 55 \% \text {. }\end{array}$ & $\begin{array}{l}\text { Critically } \\
\text { low }\end{array}$ \\
\hline $\begin{array}{l}\text { Bacigalupo } \\
\text { et al (2013) } \\
{[50]}\end{array}$ & $5(7)$ & $\begin{array}{l}\text { Narrative analysis } \\
\text { of RCTs }\end{array}$ & Mobile & $\begin{array}{l}\text { Weight loss and } \\
\text { BMI }\end{array}$ & $\begin{array}{l}9-52 \\
\text { weeks }\end{array}$ & $\begin{array}{l}\text { Strong evidence for weight loss } \\
\text { in the short term with moderate } \\
\text { evidence for the medium term. }\end{array}$ & Low \\
\hline $\begin{array}{l}\text { Covolo et al } \\
(2017)[104]\end{array}$ & $21(40)$ & $\begin{array}{l}\text { Narrative synthesis } \\
\text { of RCTs }\end{array}$ & Mobile & $\begin{array}{l}\text { BMI and waist cir- } \\
\text { cumference }\end{array}$ & $\begin{array}{l}\text { 6-12 } \\
\text { months }\end{array}$ & $\begin{array}{l}62 \%(13 / 21) \text { of studies did not } \\
\text { find a statistical difference in } \\
\text { changes in weight. } 24 \%(5 / 21) \\
\text { of studies found that a mobile } \\
\text { app was more effective compared } \\
\text { with controls }(P<.05) \text {. In } 3 \text { stud- } \\
\text { ies, this did not differ significant- } \\
\text { ly between the } 2 \text { groups. }\end{array}$ & $\begin{array}{l}\text { Critically } \\
\text { low }\end{array}$ \\
\hline $\begin{array}{l}\text { Head et al } \\
(2013)[107]\end{array}$ & $3(19)$ & $\begin{array}{l}\text { Meta-analysis of } \\
\text { RCTs }\end{array}$ & $\begin{array}{l}\text { Mobile (SMS } \\
\text { text messaging) }\end{array}$ & Weight & $\begin{array}{l}\text { Mean } \\
81.26 \\
\text { days }\end{array}$ & $\begin{array}{l}\text { The weighted mean effect size } \\
\text { for weight loss was Cohen } \\
d=0.255 \text { ( } 95 \% \text { CI } .056 \text { to } .455 \text {; } \\
P=.01 ; \mathrm{k}=3) \text {. }\end{array}$ & $\begin{array}{l}\text { Critically } \\
\text { low }\end{array}$ \\
\hline $\begin{array}{l}\text { Liu et al } \\
\text { (2015) [59] }\end{array}$ & $9(14)$ & $\begin{array}{l}\text { Meta-analysis of } \\
\text { RCTs }\end{array}$ & Mobile & Weight and BMI & $\begin{array}{l}3-30 \\
\text { months }\end{array}$ & $\begin{array}{l}\text { Compared with the control } \\
\text { group, mobile phone intervention } \\
\text { was associated with significant } \\
\text { changes in body weight and body } \\
\text { mass index (weight }[\mathrm{kg}] / \text { height } \\
\left(\mathrm{m}^{2}\right) \text { of }-1.44 \mathrm{~kg}(95 \% \mathrm{CI}-2.12 \\
\text { to }-0.76) \text { and }-0.24 \text { units ( } 95 \% \\
\mathrm{CI}-0.40 \text { to }-0.08) \text {, respectively; } \\
\text { no differences between shorter } \\
\text { and longer trials (< or } \geq 6 \text { months; } \\
\mathrm{k}=22 \text { ). }\end{array}$ & $\begin{array}{l}\text { Critically } \\
\text { low }\end{array}$ \\
\hline $\begin{array}{l}\text { Lyzwinksi et } \\
\text { al (2014) } \\
{[60]}\end{array}$ & $8(17)$ & $\begin{array}{l}\text { Meta-analysis of } \\
\text { RCTs }\end{array}$ & Mobile & $\begin{array}{l}\text { Body weight and } \\
\text { BMI }\end{array}$ & $\begin{array}{l}8 \text { weeks- } \\
12 \\
\text { months }\end{array}$ & $\begin{array}{l}75 \%(6 / 8) \text { of studies of mobile } \\
\text { phone interventions found signif- } \\
\text { icant changes in weight favoring } \\
\text { the mobile phone intervention } \\
\text { groups over the controls; the } \\
\text { meta-analysis generated a medi- } \\
\text { um, significant effect size of } \\
0.430 \text { ( } 95 \% \text { CI } 0.252 \text { to } 0.609 \text {; } \\
P \leq .01) \text {, favoring mobile interven- } \\
\text { tions. }\end{array}$ & $\begin{array}{l}\text { Critically } \\
\text { low }\end{array}$ \\
\hline
\end{tabular}




\begin{tabular}{|c|c|c|c|c|c|c|c|}
\hline Review & $\begin{array}{l}\text { Relevant } \\
\text { studies, n } \\
\text { (total } \\
\text { studies) }\end{array}$ & $\begin{array}{l}\text { Method of synthe- } \\
\text { sis }\end{array}$ & Interventions & Outcomes & $\begin{array}{l}\text { Follow- } \\
\text { up }\end{array}$ & Summary of findings & $\begin{array}{l}\text { AM- } \\
\text { STAR-2 } \\
\text { rating }\end{array}$ \\
\hline
\end{tabular}

\begin{tabular}{|c|c|c|c|c|c|}
\hline $\begin{array}{l}\text { Palmer et al } \\
\text { (2018) [111] }\end{array}$ & $3(71)$ & $\begin{array}{l}\text { Meta-analysis of } \\
\text { RCTs }\end{array}$ & Mobile & $\begin{array}{l}\text { Body weight and } \\
\text { triglyceride levels }\end{array}$ & $\begin{array}{l}24 \text { hours- } \\
6 \text { months }\end{array}$ \\
\hline
\end{tabular}

There were, at best, modest ben- Moderate

efits of diet and physical activity interventions. The effect of SMS text messaging-based diet and physical activity interventions on incidence of diabetes was pooled (risk ratio $0.67 ; 95 \% \mathrm{CI} 0.49$ to $0.90 ; \mathrm{I}^{2}=0.0 \%$ ); end point weight was pooled (MD $-0.99 \mathrm{~kg} ; 95 \%$ CI -3.63 to $1.64 ; \mathrm{I}^{2}=29.4 \%$ ); percentage change in weight was pooled (MD $-3.1 ; 95 \% \mathrm{CI}-4.86$ to $-1.3 ; \mathrm{I}^{2}=0.3 \%$ ); and triglyceride levels was pooled (MD $-0.19 \mathrm{mmol} / \mathrm{L} ; 95 \% \mathrm{CI}-0.29$ to $\left.-0.08 ; \mathrm{I}^{2}=0 \%\right)$.

\begin{tabular}{|c|c|c|c|c|c|c|c|}
\hline $\begin{array}{l}\text { Schoeppe et } \\
\text { al (2016) } \\
{[68]}\end{array}$ & $10(27)$ & $\begin{array}{l}\text { Narrative synthesis } \\
\text { of RCTs, random- } \\
\text { ized trials, con- } \\
\text { trolled trials, and } \\
\text { pre- and poststud- } \\
\text { ies }\end{array}$ & Mobile & $\begin{array}{l}\text { Physical activity; } \\
\text { diet; weight status; } \\
\text { BMI; blood pres- } \\
\text { sure; sedentary be- } \\
\text { havior; and fitness }\end{array}$ & $\begin{array}{l}1-24 \\
\text { weeks }\end{array}$ & $\begin{array}{l}40 \%(4 / 10) \text { of studies that mea- } \\
\text { sured weight reported significant } \\
\text { improvement in weight status; } \\
\text { apps were more successful when } \\
\text { used alongside other intervention } \\
\text { components than when used } \\
\text { alone. }\end{array}$ & $\begin{array}{l}\text { Critically } \\
\text { low }\end{array}$ \\
\hline $\begin{array}{l}\text { Siopis et al } \\
\text { (2015) [71] }\end{array}$ & $6(14)$ & $\begin{array}{l}\text { Meta-analysis of } \\
\text { RCTs, quasi- } \\
\text { RCTs, and pre-post } \\
\text { studies }\end{array}$ & Mobile & $\begin{array}{l}\text { Body weight and } \\
\text { BMI }\end{array}$ & $\begin{array}{l}8 \text { weeks- } \\
12 \\
\text { months }\end{array}$ & $\begin{array}{l}\text { The weighted mean change in } \\
\text { body weight in intervention par- } \\
\text { ticipants was }-2.56 \mathrm{~kg}(95 \% \mathrm{CI} \\
-3.46 \text { to }-1.65) \text { and in controls, } \\
-0.37 \mathrm{~kg}(95 \% \mathrm{CI}-1.22 \text { to } 0.48) \text {. }\end{array}$ & $\begin{array}{l}\text { Critically } \\
\text { low }\end{array}$ \\
\hline $\begin{array}{l}\text { Wieland et } \\
\text { al (2012) } \\
{[74]}\end{array}$ & $18(18)$ & $\begin{array}{l}\text { Meta-analysis of } \\
\text { RCTs, quasi- } \\
\text { RCTs, and quasiex- } \\
\text { perimental studies }\end{array}$ & Computer based & Weight & $\begin{array}{l}4 \text { weeks- } \\
30 \\
\text { months }\end{array}$ & $\begin{array}{l}\text { At } 6 \text { months, computer-based in- } \\
\text { terventions led to greater weight } \\
\text { loss than minimal interventions } \\
\text { (MD }-1.5 \mathrm{~kg} \text {; } 95 \% \mathrm{CI}-2.1 \text { to } \\
-0.9 ; 2 \text { trials) but less weight loss } \\
\text { than in-person treatment (MD } 2.1 \\
\mathrm{~kg} ; 95 \% \mathrm{CI} 0.8 \text { to } 3.4 ; 1 \text { trial). At } \\
6 \text { months, computer-based inter- } \\
\text { ventions were superior to a mini- } \\
\text { mal control intervention in limit- } \\
\text { ing weight regain (MD - } 0.7 \mathrm{~kg} \text {; } \\
95 \% \mathrm{CI}-1.2 \text { to }-0.2 ; 2 \text { trials) but } \\
\text { not superior to infrequent in-per- } \\
\text { son treatment (MD } 0.5 \mathrm{~kg} ; 95 \% \\
-0.5 \text { to } 1.6 ; 2 \text { trials). }\end{array}$ & $\begin{array}{l}\text { Critically } \\
\text { low }\end{array}$ \\
\hline $\begin{array}{l}\text { Afshin et al } \\
\text { (2016) [99] }\end{array}$ & 35 (224) & $\begin{array}{l}\text { Narrative synthesis } \\
\text { and meta-synthesis } \\
\text { for RCTs and } \\
\text { quasiexperimental } \\
\text { studies }\end{array}$ & $\begin{array}{l}\text { Various: inter- } \\
\text { net and mobile }\end{array}$ & Weight & $\begin{array}{l}3-30 \\
\text { months }\end{array}$ & $\begin{array}{l}69 \%(24 / 35) \text { of studies reported } \\
\text { significant improvements in adi- } \\
\text { posity following the intervention. } \\
81 \% \text { (13/16) of RCTs reported } \\
\text { significant reductions in adiposi- } \\
\text { ty; using the internet in the } \\
\text { weight loss program resulted in } \\
0.68 \mathrm{~kg} \text { ( } 95 \% \text { CI } 0.08 \text { to } 1.29 \mathrm{~kg}) \\
\text { additional weight reduction over } \\
\text { a period of } 3 \text { to } 30 \text { months; in } \\
\text { studies finding significant weight } \\
\text { reduction, the magnitude of } \\
\text { weight change ranged from } 1 \text { to } \\
6 \mathrm{~kg} \text { after } 6 \text { months of follow-up. }\end{array}$ & $\begin{array}{l}\text { Critically } \\
\text { low }\end{array}$ \\
\hline
\end{tabular}




\begin{tabular}{|c|c|c|c|c|c|c|c|}
\hline Review & $\begin{array}{l}\text { Relevant } \\
\text { studies, } \mathrm{n} \\
\text { (total } \\
\text { studies) }\end{array}$ & $\begin{array}{l}\text { Method of synthe- } \\
\text { sis }\end{array}$ & Interventions & Outcomes & $\begin{array}{l}\text { Follow- } \\
\text { up }\end{array}$ & Summary of findings & $\begin{array}{l}\text { AM- } \\
\text { STAR-2 } \\
\text { rating }\end{array}$ \\
\hline $\begin{array}{l}\text { Allen et al } \\
\text { (2014) [48] }\end{array}$ & $38(39)$ & $\begin{array}{l}\text { Narrative synthesis } \\
\text { of randomized tri- } \\
\text { als }\end{array}$ & $\begin{array}{l}\text { Various: inter- } \\
\text { net, messaging, } \\
\text { chat rooms, and } \\
\text { mobile }\end{array}$ & Weight loss & $\begin{array}{l}5 \text { weeks- } \\
24 \\
\text { months }\end{array}$ & $\begin{array}{l}53 \%(21 / 39) \text { of RCTs reported } \\
\text { statistically significant weight } \\
\text { loss in the intervention group as } \\
\text { compared with the control group; } \\
\text { the proportion varied by mode of } \\
\text { delivery, the highest proportion } \\
\text { of successful trials involving } \\
\text { SMS text messaging or email } \\
(67 \%) \text {, followed by online chat } \\
\text { rooms }(50 \%) \text {, web-based ( } 48 \%) \text {, } \\
\text { and self-monitoring with technol- } \\
\text { ogy }(43 \%) \text {. }\end{array}$ & $\begin{array}{l}\text { Critically } \\
\text { low }\end{array}$ \\
\hline $\begin{array}{l}\text { Bassi et al } \\
(2014) \text { [51] }\end{array}$ & $8(28)$ & $\begin{array}{l}\text { Narrative analysis } \\
\text { of RCTs }\end{array}$ & $\begin{array}{l}\text { Various: inter- } \\
\text { net and mobile }\end{array}$ & BMI and weight & $\begin{array}{l}12 \\
\text { months }\end{array}$ & $\begin{array}{l}\text { Results were mixed; } 2 \text { studies } \\
\text { reported significant improve- } \\
\text { ments with weight loss; however, } \\
\text { effects were typically short lived, } \\
\text { and more weight is regained in a } \\
\text { primarily technology-based ap- } \\
\text { proach, as compared with person- } \\
\text { al contact. }\end{array}$ & $\begin{array}{l}\text { Critically } \\
\text { low }\end{array}$ \\
\hline $\begin{array}{l}\text { Carvalho de } \\
\text { Menzes et al } \\
(2016)[53]\end{array}$ & $18(18)$ & $\begin{array}{l}\text { Narrative synthesis } \\
\text { of all study types }\end{array}$ & $\begin{array}{l}\text { Various: email, } \\
\text { telephone, face- } \\
\text { to-face, and } \\
\text { websites }\end{array}$ & $\begin{array}{l}\text { Fat consumption, } \\
\text { fruit and vegetable } \\
\text { consumption, and } \\
\text { physical activity }\end{array}$ & $\begin{array}{l}1-36 \\
\text { months }\end{array}$ & $\begin{array}{l}\text { Approximately half the studies } \\
\text { showed weight loss in the inter- } \\
\text { vention group. }\end{array}$ & $\begin{array}{l}\text { Critically } \\
\text { low }\end{array}$ \\
\hline $\begin{array}{l}\text { Coons et al } \\
(2012)[54]\end{array}$ & $13(13)$ & $\begin{array}{l}\text { Narrative synthesis } \\
\text { of RCTs }\end{array}$ & $\begin{array}{l}\text { Various: } \mathrm{PDA}^{\mathrm{f}}, \\
\text { web-based, and } \\
\text { wearables }\end{array}$ & $\begin{array}{l}\text { Body mass; BMI; } \\
\mathrm{BP}^{\mathrm{g}} \text {; waist circum- } \\
\text { ference; RHR } \\
\text { physical activity; } \\
\text { body fat percent- } \\
\text { age; energy intake; } \\
\text { and } \mathrm{EE}^{\mathrm{i}}\end{array}$ & $\begin{array}{l}12 \\
\text { weeks-24 } \\
\text { months }\end{array}$ & $\begin{array}{l}50 \%(6 / 12) \text { of weight loss trials } \\
\text { reported significantly greater } \\
\text { weight loss among individuals } \\
\text { randomized to technology inter- } \\
\text { ventions compared with controls; } \\
\text { insufficient evidence to deter- } \\
\text { mine the effectiveness of inter- } \\
\text { ventions for weight maintenance. }\end{array}$ & $\begin{array}{l}\text { Critically } \\
\text { low }\end{array}$ \\
\hline $\begin{array}{l}\text { Dutton et al } \\
\text { (2014) [55] }\end{array}$ & $18(22)$ & $\begin{array}{l}\text { Narrative synthesis } \\
\text { of all study types }\end{array}$ & $\begin{array}{l}\text { Various: mo- } \\
\text { bile, internet, } \\
\text { and podcasts }\end{array}$ & Weight & $\begin{array}{l}3 \text { weeks- } \\
24 \\
\text { months }\end{array}$ & $\begin{array}{l}67 \%(12 / 18) \text { of trials found sig- } \\
\text { nificant differences in weight } \\
\text { loss at one or more assessments. }\end{array}$ & $\begin{array}{l}\text { Critically } \\
\text { low }\end{array}$ \\
\hline $\begin{array}{l}\text { Maxwell } \\
(2015)[64]\end{array}$ & $\begin{array}{l}\text { Not re- } \\
\text { ported }\end{array}$ & $\begin{array}{l}\text { Narrative synthesis } \\
\text { of all study types }\end{array}$ & $\begin{array}{l}\text { Technology in- } \\
\text { terventions, in- } \\
\text { cluding web- } \\
\text { based and mo- } \\
\text { bile }\end{array}$ & $\begin{array}{l}\text { Healthy eating and } \\
\text { active living }\end{array}$ & $\begin{array}{l}\text { Not re- } \\
\text { ported }\end{array}$ & $\begin{array}{l}\text { Men participate in technology- } \\
\text { based healthy lifestyle interven- } \\
\text { tions less than women; mainte- } \\
\text { nance of behavior is challenging. }\end{array}$ & $\begin{array}{l}\text { Critically } \\
\text { low }\end{array}$ \\
\hline $\begin{array}{l}\text { Podina and } \\
\text { Fodor }(2018) \\
{[66]}\end{array}$ & $43(47)$ & $\begin{array}{l}\text { Meta-analysis of } \\
\text { RCTs }\end{array}$ & $\begin{array}{l}\text { Various: mobile } \\
\text { messaging, mo- } \\
\text { bile app, and } \\
\text { website }\end{array}$ & $\begin{array}{l}\text { Weight, BMI, } \\
\text { waist circumfer- } \\
\text { ence, and percent- } \\
\text { age of body fat }\end{array}$ & $\begin{array}{l}3-24 \\
\text { months }\end{array}$ & $\begin{array}{l}\text { Standard active treatment was } \\
\text { more effective than eHealth inter- } \\
\text { ventions with regard to weight } \\
\text { ( } g=-0.31 ; 95 \% \mathrm{CI}-0.43 \text { to } \\
-0.20) \text {. There was a statistically } \\
\text { significant, albeit small effect } \\
\text { size favoring eHealth interven- } \\
\text { tions relative to passive control } \\
\text { groups for weight ( } g=0.34 ; 95 \% \\
\text { CI } 0.24 \text { to } 0.44) \text { and behavioral } \\
\text { outcomes }(g=0.17 ; 95 \% \text { CI } 0.07 \\
\text { to } 0.27) \text {. }\end{array}$ & $\begin{array}{l}\text { Critically } \\
\text { low }\end{array}$ \\
\hline
\end{tabular}




\begin{tabular}{|c|c|c|c|c|c|c|c|}
\hline Review & $\begin{array}{l}\text { Relevant } \\
\text { studies, } \mathrm{n} \\
\text { (total } \\
\text { studies) }\end{array}$ & $\begin{array}{l}\text { Method of synthe- } \\
\text { sis }\end{array}$ & Interventions & Outcomes & $\begin{array}{l}\text { Follow- } \\
\text { up }\end{array}$ & Summary of findings & $\begin{array}{l}\text { AM- } \\
\text { STAR-2 } \\
\text { rating }\end{array}$ \\
\hline $\begin{array}{l}\text { Ryan et al } \\
(2019)[67]\end{array}$ & $6(6)$ & $\begin{array}{l}\text { Narrative synthesis } \\
\text { of randomized tri- } \\
\text { als }\end{array}$ & $\begin{array}{l}\text { Various: mobile } \\
\text { or internet }\end{array}$ & Weight loss & $\begin{array}{l}5 \text { weeks- } \\
24 \\
\text { months }\end{array}$ & $\begin{array}{l}\text { Tailored interventions were } \\
\text { found to be more effective in } \\
\text { supporting weight loss than } \\
\text { generic or waitlist controls in } \\
66 \%(4 / 6) \text { of articles. Effect sizes } \\
\text { were very small to moderate, } \\
\text { with evidence of fluctuations in } \\
\text { effect sizes and differences of } \\
\text { effect between tailored and non- } \\
\text { tailored interventions, and be- } \\
\text { tween tailoring types, over time. }\end{array}$ & $\begin{array}{l}\text { Critically } \\
\text { low }\end{array}$ \\
\hline
\end{tabular}

\section{Nonactive controls}

\begin{tabular}{|c|c|c|c|c|c|c|c|}
\hline $\begin{array}{l}\text { An et al } \\
(2017) \text { [49] }\end{array}$ & $21(22)$ & $\begin{array}{l}\text { Meta-analysis of } \\
\text { RCTs, pre-post } \\
\text { studies, and cohort } \\
\text { studies }\end{array}$ & Social media & $\begin{array}{l}\text { Physical activity; } \\
\text { sedentary behav- } \\
\text { ior; diet; BMI; hip- } \\
\text { waist ratio; body } \\
\text { fat; and waist cir- } \\
\text { cumference }\end{array}$ & $\begin{array}{l}2-102 \\
\text { weeks }\end{array}$ & $\begin{array}{l}\text { Social media-based interventions } \\
\text { were found to reduce body } \\
\text { weight by } 1.01 \mathrm{~kg} \text { ( } 95 \% \text { CI } 0.45 \\
\text { to } 1.57), \text { BMI by } 0.92 \mathrm{~kg} / \mathrm{m}^{2} \\
\text { ( } 95 \% \text { CI } 0.29 \text { to } 1.54) \text {, and waist } \\
\text { circumference by } 2.65 \mathrm{~cm} \text { ( } 95 \% \\
\text { CI } 0.86 \text { to } 4.43 \text { ). }\end{array}$ & $\begin{array}{l}\text { Critically } \\
\text { low }\end{array}$ \\
\hline $\begin{array}{l}\text { Tang et al } \\
(2016) \text { [72] }\end{array}$ & $18(27)$ & $\begin{array}{l}\text { Meta-analysis of } \\
\text { RCTs }\end{array}$ & Various & $\begin{array}{l}\text { Body weight; BMI; } \\
\text { and waist circum- } \\
\text { ference }\end{array}$ & $\begin{array}{l}1-24 \\
\text { months }\end{array}$ & $\begin{array}{l}\text { Participants receiving internet- } \\
\text { based, self-directed interventions } \\
\text { lost significantly more weight } \\
\text { than those receiving minimal in- } \\
\text { tervention or no treatment (MD } \\
-1.72 \mathrm{~kg} ; 95 \% \mathrm{CI}-2.60 \text { to } \\
-0.84 ; \text { significant mean differ- } \\
\text { ence }-0.45 ; 95 \% \mathrm{CI}-0.67 \text { to } \\
\left.-0.23 ; \mathrm{I}^{2}=80 \% ; P<.001\right) \text { and a } \\
\text { significantly greater reduction in } \\
\text { BMI levels than those receiving } \\
\text { no treatment or minimal interven- } \\
\text { tion }\left(\mathrm{MD}-0.47 \mathrm{~kg} / \mathrm{m}^{2} ; 95 \% \mathrm{CI}\right. \\
-0.81 \text { to }-0.14 ; \text { significant mean } \\
\text { difference }-0.32 ; 95 \% \mathrm{CI}-0.61 \\
\text { to }-0.03 ; \mathrm{I}^{2}=90 \% ; P=.03 ; 13 \\
\text { evaluations). There was a greater } \\
\text { reduction in BMI (MD } 0.54 \\
\left.\mathrm{~kg} / \mathrm{m}^{2}\right) \text { and waist circumference } \\
(2.81 \mathrm{~cm}) \text { at } 0-4 \text { months follow- } \\
\text { up than at later times }(\mathrm{k}=27) \text {. }\end{array}$ & $\begin{array}{l}\text { Critically } \\
\text { low }\end{array}$ \\
\hline \multicolumn{8}{|l|}{ tive controls } \\
\hline $\begin{array}{l}\text { Beleigoli et } \\
\text { al (2019) } \\
{[52]}\end{array}$ & $11(11)$ & $\begin{array}{l}\text { Meta-analysis of } \\
\text { RCTs }\end{array}$ & Internet & Weight and BMI & $\begin{array}{l}3-12 \\
\text { months }\end{array}$ & $\begin{array}{l}\text { Compared with offline interven- } \\
\text { tions, digital interventions led to } \\
\text { a greater short-term (<6 months } \\
\text { follow-up) weight loss (MD } \\
-2.13 \mathrm{~kg} ; 95 \% \mathrm{CI}-2.71 \text { to } \\
-1.55 ; 393 \text { participants; high- } \\
\text { certainty evidence) but not in the } \\
\text { long-term (MD - } 0.17 \mathrm{~kg} \text {; } 95 \% \\
\mathrm{CI}-2.10 \text { to } 1.76 ; 1104 \text { partici- } \\
\text { pants; moderate-certainty evi- } \\
\text { dence). }\end{array}$ & $\begin{array}{l}\text { Critically } \\
\text { low }\end{array}$ \\
\hline
\end{tabular}




\begin{tabular}{|c|c|c|c|c|c|c|c|}
\hline Review & $\begin{array}{l}\text { Relevant } \\
\text { studies, n } \\
\text { (total } \\
\text { studies) }\end{array}$ & $\begin{array}{l}\text { Method of synthe- } \\
\text { sis }\end{array}$ & Interventions & Outcomes & $\begin{array}{l}\text { Follow- } \\
\text { up }\end{array}$ & Summary of findings & $\begin{array}{l}\text { AM- } \\
\text { STAR-2 } \\
\text { rating }\end{array}$ \\
\hline $\begin{array}{l}\text { Kodama et } \\
\text { al (2012) } \\
{[58]}\end{array}$ & $23(23)$ & $\begin{array}{l}\text { Meta-analysis of } \\
\text { RCTs }\end{array}$ & Internet & Weight loss & $\begin{array}{l}3-30 \\
\text { months }\end{array}$ & $\begin{array}{l}\text { Using the internet had a modest } \\
\text { but significant additional weight } \\
\text { loss effect compared with non- } \\
\text { web user control groups ( }-0.68 \\
\mathrm{~kg} ; P=.03 \text { ). Internet-based inter- } \\
\text { ventions were effective for } \\
\text { weight loss ( }-1.00 \mathrm{~kg} ; P<.001) \\
\text { but not a substitute for face-to- } \\
\text { face support ( }+1.27 \mathrm{~kg} ; P=.01) \text {. } \\
\text { An additional effect on weight } \\
\text { control was observed when the } \\
\text { aim of using the internet was } \\
\text { initial weight loss }(-1.01 \mathrm{~kg} ; \\
P=.03) \text { but was not observed } \\
\text { when the aim was weight mainte- } \\
\text { nance ( }+0.68 \mathrm{~kg} ; P=.26) \text {; further- } \\
\text { more, it was effective to use the } \\
\text { internet as an adjunct to face-to- } \\
\text { face care }(-1.00 \mathrm{~kg} ; P<.001) \text { but } \\
\text { adverse effects on weight loss } \\
\text { were found when it was used as } \\
\text { a substitute ( }+1.27 \mathrm{~kg} ; P=.01) \text {. } \\
\text { The weight loss effect was in- } \\
\text { significant ( }-0.20 \mathrm{~kg} ; P=.75) \text { in } \\
\text { studies with educational periods } \\
\geq 12 \text { months and was significant } \\
\text { in studies with an educational } \\
\text { period }<6 \text { months ( }-1.55 \mathrm{~kg} \text {; } \\
P=.001) \text {. }\end{array}$ & $\begin{array}{l}\text { Critically } \\
\text { Low }\end{array}$ \\
\hline
\end{tabular}

${ }^{\mathrm{a}} \mathrm{RCT}$ : randomized controlled trial.

${ }^{\mathrm{b}} \mathrm{HbA}_{1 \mathrm{c}}$ : glycated hemoglobin.

${ }^{\mathrm{c}}$ LDL: low-density lipoprotein.

${ }^{\mathrm{d}} \mathrm{MD}$ : mean difference.

${ }^{\text {e }}$ SMD: standardized mean difference.

${ }^{\mathrm{f}}$ PDA: personal digital assistant.

$\mathrm{g}_{\mathrm{BP}}$ : blood pressure.

${ }^{\mathrm{h}} \mathrm{RHR}$ : resting heart rate.

${ }^{\mathrm{i}} \mathrm{EE}$ : energy expenditure.

Internet interventions were found to be somewhat effective. A total of 5 narrative syntheses found them to be effective in approximately half of the studies $[47,62,99,100,108]$. In total, 3 meta-analyses quantified weight loss and other health effects. A total of 3 reviews found a significant reduction in weight: mean difference $-1.34 \mathrm{~kg}$ [102], SMD $2.13 \mathrm{~kg}$ [70], and a $0.68-\mathrm{kg}$ additional weight reduction over a period of 3 to 30 months [99]. A fourth review found that internet interventions significantly reduced waist circumference (mean change -2.99 cm) [69]. However, stratified analysis suggested that internet interventions were effective when used in combination with in-person counseling $(-1.93 \mathrm{~kg})$, rather than as a substitute [98]. This finding was supported by a narrative synthesis, which reported that internet interventions were more effective when they were enhanced to offer more than just educational resources (several studies found medium effect sizes) [62].

There were mixed results regarding the effectiveness of social media interventions. A total of 2 reviews were favorable $[49,56]$, including a meta-analysis that found that social media-based interventions reduced body weight by $1.01 \mathrm{~kg}$, BMI by 0.92 $\mathrm{kg} / \mathrm{m}^{2}$, and waist circumference by $2.65 \mathrm{~cm}$ but did not find significant changes in body fat or body fat percentage [49]. A total of 2 narrative syntheses found effects that were small and not meaningful $[61,76]$. Two meta-analyses found no significant effects on the diverse primary outcomes of the studies or on body weight $[65,75]$.

Most reviews of mobile phone interventions found that they were effective in achieving weight loss via diet and physical activity. One meta-analysis found a statistically significant medium effect size (Cohen $d=0.430$ ) in favor of mobile phone interventions [60]. Two others quantified the change in terms of body weight: mobile phone interventions were associated with significant changes in body weight of $-1.44 \mathrm{~kg}$ and in BMI of $-0.24 \mathrm{~kg} / \mathrm{m}^{2}$ compared with controls [59]; the weighted mean body weight change in intervention participants was $-2.56 \mathrm{~kg}$ compared with $-0.37 \mathrm{~kg}$ in controls [71]. These results were supported by 3 narrative syntheses, which found evidence that 
mobile interventions led to weight loss $[50,68,99]$. However, one review of apps to promote healthy lifestyles found that most trials did not show significant differences [104], and one of the reviews that looked at the use of apps for diet, physical activity, and sedentary behavior, which concluded that apps led to weight loss, also concluded that the apps were more successful when used alongside other intervention components than when used alone [68].

In total, 4 of 5 narrative syntheses that ranged over a variety of digital interventions found that around half of the interventions were effective compared with controls [48,51,53,54], with only one finding that a large majority $(81 \%)$ reported a significant reduction in adiposity [99]. One of these studies found that most trials reported within-group weight loss, even when there was no difference between digital interventions and controls [54]. Another study compared the success of different modes of delivery, finding the highest proportion of successful trials involving SMS text messaging or email (67\%), followed by online chat rooms (50\%), web-based (48\%), and self-monitoring with technology (43\%) [48].

\section{Effectiveness of Digital Interventions on Diet and Physical Activity (Weight Loss) Compared With Nonactive Intervention Controls}

A total of 6 reviews that reported results for digital interventions for diet and physical activity to no intervention or minimal intervention controls all agreed that digital interventions were more effective $[66,67,69,70,72,74]$. Table 5 provides a summary of the results.

Internet interventions were more effective than nonactive controls according to 3 meta-analyses and 1 narrative synthesis $[67,69,70,72]$. Internet interventions led to a greater reduction in waist circumference (mean change $-2.99 \mathrm{~cm}, 95 \% \mathrm{CI}-3.68$ to $-2.30, \mathrm{I} 2=93.3 \%$ vs $2.38 \mathrm{~cm}, 95 \%$ CI 1.61 to 3.25 , I2=97.2\%) [69]; internet-delivered personal feedback led to greater weight loss (mean difference $2.14 \mathrm{~kg}$ ) [70]; and self-directed internet interventions led to significantly more weight loss (mean difference $-1.56 \mathrm{~kg}$ ) and showed a significantly greater reduction in BMI (mean difference $-0.41 \mathrm{~kg} / \mathrm{m}^{2}$ ) [72].

Similar effects were observed for computer-based interventions, which led to greater weight loss at 6 months (mean difference $-1.5 \mathrm{~kg}$ ) and were superior to limiting weight regain (mean difference $-0.7 \mathrm{~kg}$ ) [74].

A narrative review of tailored internet interventions found that effect sizes ranged from very small to moderate [67]. This was supported by a meta-analysis that covered various digital interventions, which found small effect sizes favoring digital interventions for weight $(g=0.34)$ and behavioral outcomes $(g=0.17)[66]$.

\section{Effectiveness of Digital Interventions on Diet and Physical Activity (Weight Loss) Compared With Active Controls}

The effects of digital interventions on diet and physical activity (with regard to weight loss) compared with active controls were mixed. A total of 2 meta-analyses found no differences between web-based interventions and active offline interventions for weight loss outcomes [52,69]. One meta-analysis of a range of interventions found that standard active treatment was more effective for weight loss $(g=0.31)$ [66]. However, one meta-analysis found that using the internet had a modest but significant additional weight loss effect compared with offline control groups $(-0.68 \mathrm{~kg} ; P=.03)$, with a subgroup analysis showing that the internet was effective compared with controls for achieving weight loss (weight change $=-1.01 \mathrm{~kg}$ ) but not weight maintenance [58]. The same meta-analysis also found that it was effective to use the internet as an adjunct to face-to-face care $(-1 \mathrm{~kg} ; P<.001)$ but that adverse effects on weight loss were found when it was used as a substitute $(+1.27$ $\mathrm{kg} ; P=.01$ ) [58].

Computer-based interventions led to less weight loss than in-person treatment (mean difference $2.1 \mathrm{~kg}$ ) and were not superior to infrequent in-person treatment in limiting weight regain at 6 months [74].

\section{Sustainability of Effects on Diet and Physical Activity (Weight Loss) at Follow-Up}

Although technology-related health interventions may be effective, the maintenance of behavior is challenging [64], and there is insufficient evidence to determine the effectiveness of digital interventions on weight maintenance [54]. One review of internet interventions reported that, in studies finding significant weight reduction, the magnitude of weight change ranged from 1 to $6 \mathrm{~kg}$ after 6 months of follow-up [99].

Many studies included in the reviews had short follow-ups. Where longer follow-ups were reported, effectiveness typically diminished over time. Examples of the diminishing effects are clear in the 2 reviews. One meta-analysis of internet-delivered personal feedback found a greater reduction in BMI (mean difference $0.54 \mathrm{~kg} / \mathrm{m}^{2}$ ) and waist circumference $(2.81 \mathrm{~cm}$ ) at 0 to 4 months follow-up than at later times compared with undefined control groups [72]. A narrative synthesis supports these findings, concluding from multiple high-quality randomized controlled trials (RCTs) that weight loss occurs for a short term through mobile interventions, with moderate evidence for the medium term [50]. Two other meta-analyses reported significantly greater weight loss in favor of digital interventions in the medium term only ( $<6$ months): $2.13 \mathrm{~kg}$ [52] and $1.55 \mathrm{~kg}$ [58]. One meta-analysis assessed differences between trials of different lengths, reporting no differences between shorter and longer trials ( $<6$ or $\geq 6$ months) [59].

\section{Smoking}

\section{Review Characteristics}

There were 28 reviews on smoking (see Multimedia Appendix 6 for summary characteristics).

\section{Effectiveness of Digital Interventions on Smoking Compared With Mixed (Active and Nonactive) Controls}

Digital interventions were generally effective across different modes of delivery (Table 6). 
Table 6. Results of reviews on smoking, ordered by type of control and further ordered by mode of delivery of intervention.

\begin{tabular}{|c|c|c|c|c|c|c|c|}
\hline Review & $\begin{array}{l}\text { Relevant } \\
\text { studies, } \mathrm{n} \\
\text { (total } \\
\text { studies) }\end{array}$ & $\begin{array}{l}\text { Method of synthe- } \\
\text { sis }\end{array}$ & Interventions & Outcomes & $\begin{array}{l}\text { Follow- } \\
\text { up }\end{array}$ & Summary of findings & $\begin{array}{l}\text { AMSTAR- } \\
2 \text { rating }\end{array}$ \\
\hline \multicolumn{8}{|c|}{ Mixed (active and nonactive) controls } \\
\hline $\begin{array}{l}\text { Afshin et al } \\
(2016) \text { [99] }\end{array}$ & $22(224)$ & $\begin{array}{l}\text { Narrative synthesis } \\
\text { of RCTs }{ }^{\mathrm{a}} \text { and } \\
\text { quasiexperimental } \\
\text { studies }\end{array}$ & $\begin{array}{l}\text { Internet and } \\
\text { mobile inter- } \\
\text { ventions }\end{array}$ & Abstinence & $\begin{array}{l}1 \text { week- } \\
2 \text { years }\end{array}$ & $\begin{array}{l}77 \%(17 / 22) \text { of studies reported } \\
\text { a significant increase in absti- } \\
\text { nence. In studies reporting bene- } \\
\text { fits, the } \mathrm{OR}^{\mathrm{b}} \text { for } 7 \text {-day abstinence } \\
\text { at } 6 \text { months ranged from } 1.6 \\
\text { (95\% CI } 1.1 \text { to } 2.4) \text { to } 2.7(95 \% \\
\text { CI } 1.8 \text { to } 4.0) \text {. }\end{array}$ & $\begin{array}{l}\text { Critically } \\
\text { low }\end{array}$ \\
\hline $\begin{array}{l}\text { Boland et al } \\
(2016)[83] \\
\text { (Smoking on- } \\
\text { ly) }\end{array}$ & $13(13)$ & $\begin{array}{l}\text { Meta-analysis of } \\
\text { RCTs }\end{array}$ & $\begin{array}{l}\text { Various: web- } \\
\text { site or comput- } \\
\text { er program } \\
\text { and SMS text } \\
\text { messaging }\end{array}$ & Abstinence & $\begin{array}{l}1 \text { week- } \\
18 \\
\text { months }\end{array}$ & $\begin{array}{l}\text { Interventions increased the odds } \\
\text { of smoking cessation for disad- } \\
\text { vantaged groups at } 1 \text { month (OR } \\
1.70 ; 95 \% \text { CI } 1.10 \text { to } 2.63), 3 \\
\text { months (OR } 1.30 ; 95 \% \text { CI } 1.07 \\
\text { to } 1.59), 6 \text { months (OR } 1.29 \text {, } \\
95 \% \text { CI } 1.03 \text { to } 1.62 \text { ) and } 18 \\
\text { months postintervention (OR } \\
1.83,95 \% \text { CI } 1.11 \text { to } 3.01) \text {. }\end{array}$ & Low \\
\hline $\begin{array}{l}\text { Aneni et al } \\
(2014)[100]\end{array}$ & $3(29)$ & $\begin{array}{l}\text { Narrative synthesis } \\
\text { of RCTs }\end{array}$ & Internet & Cessation & $\begin{array}{l}12 \\
\text { months }\end{array}$ & $\begin{array}{l}3 \text { follow-up studies that mea- } \\
\text { sured smoking cessation showed } \\
\text { significant intervention effects, } \\
\text { although they were assessed to } \\
\text { be of low quality. }\end{array}$ & $\begin{array}{l}\text { Critically } \\
\text { low }\end{array}$ \\
\hline $\begin{array}{l}\text { Chebli et al } \\
(2016) \text { [103] }\end{array}$ & $9(16)$ & $\begin{array}{l}\text { Narrative synthesis } \\
\text { of RCTs }\end{array}$ & Internet & $\begin{array}{l}\text { Cessation and re- } \\
\text { duction }\end{array}$ & $\begin{array}{l}1-12 \\
\text { months }\end{array}$ & $\begin{array}{l}\text { Internet-based interventions may } \\
\text { have a positive effect on smoking } \\
\text { cessation. Several studies found } \\
\text { that web-based use and number } \\
\text { of log-ins was positively associ- } \\
\text { ated with quit outcomes. }\end{array}$ & $\begin{array}{l}\text { Critically } \\
\text { low }\end{array}$ \\
\hline $\begin{array}{l}\text { Cheung et al } \\
(2017)[85] \\
\text { (Smoking on- } \\
\text { ly) }\end{array}$ & $6(45)$ & $\begin{array}{l}\text { Narrative synthesis } \\
\text { and meta-analysis } \\
\text { of RCTs and quasi- } \\
\text { RCTs }\end{array}$ & Internet & Cessation & $\begin{array}{l}>4 \\
\text { weeks }\end{array}$ & $\begin{array}{l}\text { Only } 13 \%(6 / 45) \text { of studies pro- } \\
\text { vided data on effectiveness, with } \\
66 \%(4 / 6) \text { of studies demonstrat- } \\
\text { ing effectiveness. Smokers using } \\
\text { a web-based cessation interven- } \\
\text { tion were } 1.15 \text { to } 2.84 \text { times more } \\
\text { likely to become a former smok- } \\
\text { er compared with the control } \\
\text { condition (with a pooled } \mathrm{RR}^{\mathrm{c}} \\
1.39 ; 95 \% \text { CI } 1.18 \text { to } 1.65) \text {. }\end{array}$ & $\begin{array}{l}\text { Critically } \\
\text { low }\end{array}$ \\
\hline $\begin{array}{l}\text { Gainsbury and } \\
\text { Blaszczynski } \\
(2010)[87] \\
\text { (Smoking on- } \\
\text { ly) }\end{array}$ & $7(9)$ & $\begin{array}{l}\text { Narrative synthesis } \\
\text { of RCTs and pre- } \\
\text { experimental stud- } \\
\text { ies }\end{array}$ & Internet & $\begin{array}{l}\text { Abstinence, tobac- } \\
\text { co use, smoking } \\
\text { status, compliance, } \\
\text { nicotine depen- } \\
\text { dence, carbon } \\
\text { monoxide markers, } \\
\text { and toxicity }\end{array}$ & $\begin{array}{l}3-12 \\
\text { months }\end{array}$ & $\begin{array}{l}86 \%(6 / 7) \text { of studies reported } \\
\text { significantly greater self-reported } \\
\text { smoking quit rates or abstinence } \\
\text { at the end of the treatment trial } \\
\text { for participants in the internet } \\
\text { intervention compared with con- } \\
\text { trols. Several trials found im- } \\
\text { provements at } 3,6 \text {, and } 12 \\
\text { months. }\end{array}$ & $\begin{array}{l}\text { Critically } \\
\text { low }\end{array}$ \\
\hline $\begin{array}{l}\text { Graham et al } \\
(2016)[88] \\
\text { (Smoking on- } \\
\text { ly) }\end{array}$ & $40(40)$ & $\begin{array}{l}\text { Meta-analysis of } \\
\text { RCTs }\end{array}$ & Internet & Abstinence & $\begin{array}{l}7 \text { days- } 3 \\
\text { months }\end{array}$ & $\begin{array}{l}\text { Pooled results from } 15 \text { trials ( } 24 \\
\text { comparisons) found a significant } \\
\text { effect in favor of experimental } \\
\text { internet interventions (RR } 1.16 \text {; } \\
95 \% \text { CI } 1.03 \text { to } 1.31 ; \mathrm{I}^{2}=76.7 \% \text { ). }\end{array}$ & $\begin{array}{l}\text { Critically } \\
\text { low }\end{array}$ \\
\hline
\end{tabular}




\begin{tabular}{|c|c|c|c|c|c|c|c|}
\hline Review & $\begin{array}{l}\text { Relevant } \\
\text { studies, } \mathrm{n} \\
\text { (total } \\
\text { studies) }\end{array}$ & $\begin{array}{l}\text { Method of synthe- } \\
\text { sis }\end{array}$ & Interventions & Outcomes & $\begin{array}{l}\text { Follow- } \\
\text { up }\end{array}$ & Summary of findings & $\begin{array}{l}\text { AMSTAR- } \\
2 \text { rating }\end{array}$ \\
\hline $\begin{array}{l}\text { Hutton et al } \\
(2011)[90] \\
\text { (Smoking on- } \\
\text { ly) }\end{array}$ & $15(21)$ & $\begin{array}{l}\text { Narrative synthesis } \\
\text { of RCTs }\end{array}$ & Internet & Cessation & $\begin{array}{l}>1 \\
\text { month }\end{array}$ & $\begin{array}{l}\text { Two RCTs found that a multi- } \\
\text { component intervention with web } \\
\text { and nonweb-based elements was } \\
\text { more efficacious than a self-help } \\
\text { manual, and one of the } 2 \text { RCTs } \\
\text { found that web-based interven- } \\
\text { tions may be more effective than } \\
\text { no treatment. Three trials provid- } \\
\text { ed insufficient evidence to } \\
\text { demonstrate whether web-based } \\
\text { interventions were more effica- } \\
\text { cious than counselling. Tailored } \\
\text { websites in } 2 \text { RCTs and greater } \\
\text { website exposure in } 86 \% \text { (6/7) } \\
\text { of RCTs were associated with } \\
\text { higher rates of abstinence. }\end{array}$ & $\begin{array}{l}\text { Critically } \\
\text { low }\end{array}$ \\
\hline $\begin{array}{l}\text { Lustria et al } \\
(2013)[110]\end{array}$ & $8(40)$ & $\begin{array}{l}\text { Meta-analysis of } \\
\text { experimental and } \\
\text { quasiexperimental } \\
\text { studies }\end{array}$ & Internet & Abstinence & $\begin{array}{l}30 \text { days- } \\
6 \\
\text { months }\end{array}$ & $\begin{array}{l}\text { Web - based, tailored interven- } \\
\text { tions had significantly greater } \\
\text { improvement in smoking out- } \\
\text { comes compared with control } \\
\text { conditions, with small effects, } \\
\text { Cohen } d=0.151(\mathrm{k}=8 ; 95 \% \mathrm{CI} \\
0.11 \text { to } 0.19 ; P<.001) \text {. }\end{array}$ & $\begin{array}{l}\text { Critically } \\
\text { low }\end{array}$ \\
\hline $\begin{array}{l}\text { McCrabb et al } \\
(2019)[91] \\
\text { (Smoking on- } \\
\text { ly) }\end{array}$ & $45(45)$ & $\begin{array}{l}\text { Meta-analysis of } \\
\text { RCTs }\end{array}$ & Internet & Abstinence & $\begin{array}{l}1-18 \\
\text { months }\end{array}$ & $\begin{array}{l}\text { Interventions were effective in } \\
\text { the short term (OR } 1.29,95 \% \text { CI } \\
1.12 \text { to } 1.50 ; P=.001) \text { and long } \\
\text { term (OR } 1.1 .9,95 \% \text { CI } 1.06 \text { to } \\
1.35 ; P=.004) \text {. }\end{array}$ & Low \\
\hline $\begin{array}{l}\text { Shahab and } \\
\text { McEwen } \\
\text { (2009) [95] } \\
\text { (Smoking on- } \\
\text { ly) }\end{array}$ & $10(11)$ & $\begin{array}{l}\text { Meta-analysis of } \\
\text { RCTs }\end{array}$ & Internet & Cessation & $\begin{array}{l}>1 \\
\text { month }\end{array}$ & $\begin{array}{l}\text { Interactive interventions were } \\
\text { effective compared with untai- } \\
\text { lored booklets or emails (RR 1.8; } \\
95 \% \text { CI } 1.4 \text { to } 2.3 \text { ) increasing 6- } \\
\text { month abstinence by } 17 \% \text { (95\% } \\
\text { CI } 12 \text { to } 21 \% \text { ); no evidence was } \\
\text { found of a difference between } \\
\text { interactive and static interven- } \\
\text { tions. }\end{array}$ & $\begin{array}{l}\text { Critically } \\
\text { low }\end{array}$ \\
\hline $\begin{array}{l}\text { Taylor et al } \\
(2017) \text { [97] } \\
\text { (Smoking on- } \\
\text { ly) }\end{array}$ & $61(67)$ & $\begin{array}{l}\text { Meta-analysis of } \\
\text { RCTs and quasi- } \\
\text { RCTs }\end{array}$ & Internet & Cessation & $\begin{array}{l}\text { 6-12 } \\
\text { months }\end{array}$ & $\begin{array}{l}\text { Interactive and tailored internet- } \\
\text { based interventions with or } \\
\text { without additional behavioral } \\
\text { support are moderately more ef- } \\
\text { fective than nonactive controls } \\
\text { at } 6 \text { months or longer, but there } \\
\text { was no evidence that these inter- } \\
\text { ventions were better than other } \\
\text { active smoking treatments. }\end{array}$ & Moderate \\
\hline $\begin{array}{l}\text { Webb et al } \\
(2010)[113]\end{array}$ & $12(85)$ & $\begin{array}{l}\text { Meta-analysis of } \\
\text { RCTs }\end{array}$ & Internet & $\begin{array}{l}\text { Smoking absti- } \\
\text { nence }\end{array}$ & $\begin{array}{l}12 \\
\text { months }\end{array}$ & $\begin{array}{l}\text { Interventions that targeted } \\
\text { smoking abstinence tended to } \\
\text { have small effects on behavior } \\
\text { that did not reach statistical sig- } \\
\text { nificance (Cohen } d+=0.07 ; \mathrm{k}=12 \text {; } \\
95 \% \mathrm{CI}-0.04 \text { to } 0.18 \text { ). }\end{array}$ & $\begin{array}{l}\text { Critically } \\
\text { low }\end{array}$ \\
\hline $\begin{array}{l}\text { Head et al } \\
(2013)[107]\end{array}$ & $5(19)$ & $\begin{array}{l}\text { Meta-analysis of } \\
\text { RCTs }\end{array}$ & $\begin{array}{l}\text { SMS text mes- } \\
\text { saging }\end{array}$ & Smoking cessation & $\begin{array}{l}\text { Mean } \\
81.26 \\
\text { days }\end{array}$ & $\begin{array}{l}\text { The weighted mean effect size } \\
\text { for smoking cessation, Cohen } \\
d=0.447(95 \% \text { CI } .367 \text { to } .526 \text {; } \\
P=.001 ; \mathrm{k}=5) \text {. }\end{array}$ & $\begin{array}{l}\text { Critically } \\
\text { low }\end{array}$ \\
\hline
\end{tabular}




\begin{tabular}{|c|c|c|c|c|c|c|c|}
\hline Review & $\begin{array}{l}\text { Relevant } \\
\text { studies, } \mathrm{n} \\
\text { (total } \\
\text { studies) }\end{array}$ & $\begin{array}{l}\text { Method of synthe- } \\
\text { sis }\end{array}$ & Interventions & Outcomes & $\begin{array}{l}\text { Follow- } \\
\text { up }\end{array}$ & Summary of findings & $\begin{array}{l}\text { AMSTAR- } \\
2 \text { rating }\end{array}$ \\
\hline $\begin{array}{l}\text { Scott-Sheldon } \\
\text { et al (2016) } \\
\text { [94] (smoking } \\
\text { only) }\end{array}$ & $18(20)$ & $\begin{array}{l}\text { Meta-analysis of } \\
\text { RCTs }\end{array}$ & $\begin{array}{l}\text { SMS text mes- } \\
\text { saging }\end{array}$ & $\begin{array}{l}\text { Abstinence, } \\
\text { cigarette use, quit } \\
\text { attempts, and nico- } \\
\text { tine dependence }\end{array}$ & $\begin{array}{l}\text { Not re- } \\
\text { ported }\end{array}$ & $\begin{array}{l}\text { SMS text messaging was associ- } \\
\text { ated with significantly greater } \\
\text { odds of abstinence compared } \\
\text { with controls: } 7 \text {-day point preva- } \\
\text { lence (OR } 1.38,95 \% \text { CI } 1.22 \text { to } \\
1.55 ; \mathrm{k}=16) \text { and continuous absti- } \\
\text { nence (OR } 1.63,95 \% \text { CI } 1.19 \text { to } \\
2.24 \text {; k=7); interventions were } \\
\text { also more successful in reducing } \\
\text { cigarette consumption (Cohen } \\
d_{+}=0.14 ; 95 \% \text { CI } 0.05 \text { to } 0.23 \text {; } \\
\mathrm{k}=9) \text {. }\end{array}$ & $\begin{array}{l}\text { Critically } \\
\text { low }\end{array}$ \\
\hline $\begin{array}{l}\text { Spohr et al } \\
\text { (2015) [96] } \\
\text { (smoking on- } \\
\text { ly) }\end{array}$ & $10(13)$ & $\begin{array}{l}\text { Meta-analysis of } \\
\text { RCTs }\end{array}$ & $\begin{array}{l}\text { SMS text mes- } \\
\text { saging }\end{array}$ & Cessation & $\begin{array}{l}3 \text { and } 6 \\
\text { months }\end{array}$ & $\begin{array}{l}\text { Interventions generally increased } \\
\text { quit rates compared with controls } \\
\text { (OR } 1.36,95 \% \text { CI } 1.23 \text { to } 1.51 \text { ). } \\
\text { Intervention efficacy was higher } \\
\text { in studies with a 3-month follow- } \\
\text { up compared with a 6-month } \\
\text { follow-up. }\end{array}$ & $\begin{array}{l}\text { Critically } \\
\text { low }\end{array}$ \\
\hline $\begin{array}{l}\text { Palmer et al } \\
(2018)[111]\end{array}$ & $18(71)$ & $\begin{array}{l}\text { Meta-analysis of } \\
\text { RCTs }\end{array}$ & Mobile & $\begin{array}{l}\text { Abstinence and } \\
\text { cessation (verified } \\
\text { biochemically) }\end{array}$ & $\begin{array}{l}24 \\
\text { hours-6 } \\
\text { months }\end{array}$ & $\begin{array}{l}\text { The effect of SMS text messag- } \\
\text { ing-based smoking cessation } \\
\text { support on biochemically veri- } \\
\text { fied continuous abstinence was } \\
\text { pooled relative risk, RR } 2.19 \\
\left.\text { (95\% CI } 1.80 \text { to } 2.68 ; \mathrm{I}^{2}=0 \%\right) \\
\text { and on verified } 7 \text {-day point } \\
\text { prevalence of smoking cessation } \\
\text { was pooled RR } 1.51(95 \% \text { CI } \\
\left.1.06 \text { to } 2.15 ; \mathrm{I}^{2}=0 \%\right) \text {. }\end{array}$ & Moderate \\
\hline $\begin{array}{l}\text { Whittaker et al } \\
(2016) \text { [98] } \\
\text { (smoking on- } \\
\text { ly) }\end{array}$ & $12(12)$ & $\begin{array}{l}\text { Meta-analysis of } \\
\text { RCTs and quasi- } \\
\text { RCTs }\end{array}$ & Mobile & Cessation & $\begin{array}{l}6 \\
\text { months }\end{array}$ & $\begin{array}{l}\text { Smokers who received support } \\
\text { programs were } 1.7 \text { times more } \\
\text { likely to stay quit than smokers } \\
\text { who did not receive the programs } \\
\text { ( } 9.3 \% \text { quit with programs com- } \\
\text { pared with } 5.6 \% \text { who quit with } \\
\text { no programs). Most of the studies } \\
\text { were of programs relying mainly } \\
\text { on text messages. }\end{array}$ & Moderate \\
\hline $\begin{array}{l}\text { Danielsson et } \\
\text { al (2014) [86] } \\
\text { (smoking on- } \\
\text { ly) }\end{array}$ & $21(74)$ & $\begin{array}{l}\text { Narrative synthesis } \\
\text { of RCTs }\end{array}$ & Various & Abstinence & $\begin{array}{l}>3 \\
\text { months }\end{array}$ & $\begin{array}{l}\text { The studies showed mixed results } \\
\text { regarding internet interventions } \\
\text { and smoking, with some positive } \\
\text { effects for the smoking cessation } \\
\text { program that combined the use } \\
\text { of both the internet, mobile } \\
\text { phones (SMS text messaging), } \\
\text { and email. }\end{array}$ & $\begin{array}{l}\text { Critically } \\
\text { low }\end{array}$ \\
\hline
\end{tabular}




\begin{tabular}{|c|c|c|c|c|c|c|c|}
\hline Review & $\begin{array}{l}\text { Relevant } \\
\text { studies, } \mathrm{n} \\
\text { (total } \\
\text { studies) }\end{array}$ & $\begin{array}{l}\text { Method of synthe- } \\
\text { sis }\end{array}$ & Interventions & Outcomes & $\begin{array}{l}\text { Follow- } \\
\text { up }\end{array}$ & Summary of findings & $\begin{array}{l}\text { AMSTAR- } \\
2 \text { rating }\end{array}$ \\
\hline $\begin{array}{l}\text { HIQA (2017) } \\
\text { [89] (smoking } \\
\text { only) }\end{array}$ & $12(143)^{\mathrm{d}}$ & $\begin{array}{l}\text { Network meta- } \\
\text { analyses of RCTs }\end{array}$ & $\begin{array}{l}\text { Various: inter- } \\
\text { net and mobile }\end{array}$ & Cessation & $\begin{array}{l}6-12 \\
\text { months }\end{array}$ & $\begin{array}{l}\text { Internet-based interventions are } \\
\text { superior to control (brief advice } \\
\text { or written materials; RR } 1.43 \text {, } \\
95 \% \text { CI } 1.02 \text { to } 2.00 ; P=.04 ; \\
\text { k=5); Internet-based interven- } \\
\text { tions are superior to doing noth- } \\
\text { ing (RR } 1.46,95 \% \text { CI } 1.18 \text { to } \\
1.81 ; P<.001 ; \mathrm{k}=3) \text {; mobile } \\
\text { phone-based interventions ap- } \\
\text { pear to have similar effectiveness } \\
\text { to control (RR } 1.18,95 \% \text { CI } 0.88 \\
\text { to } 1.60 ; P=.27 ; \mathrm{k}=3) \text {; no evidence } \\
\text { of difference between mobile } \\
\text { phone-based interventions and } \\
\text { internet }(\mathrm{RR} 1.43,95 \% \text { CI } 0.88 \\
\text { to } 2.31 ; P=.15 ; \mathrm{k}=1) \text {. }\end{array}$ & Low \\
\hline $\begin{array}{l}\text { Hou et al } \\
(2013)[108]\end{array}$ & $5(38)$ & $\begin{array}{l}\text { Narrative synthesis } \\
\text { of studies with } \\
\text { comparison or con- } \\
\text { trol groups }\end{array}$ & $\begin{array}{l}\text { Web-based } \\
\text { computer pro- } \\
\text { grams }\end{array}$ & Cessation & $\begin{array}{l}\text { Not re- } \\
\text { ported }\end{array}$ & $\begin{array}{l}2 \text { studies found higher cessation } \\
\text { rates in intervention groups than } \\
\text { control. } 3 \text { studies found no signif- } \\
\text { icant differences in quit rates at } \\
\text { the end of the intervention or at } \\
\text { follow-ups. }\end{array}$ & $\begin{array}{l}\text { Critically } \\
\text { low }\end{array}$ \\
\hline $\begin{array}{l}\text { Myung et al } \\
\text { (2009) [92] } \\
\text { (smoking on- } \\
\text { ly) }\end{array}$ & $22(22)$ & $\begin{array}{l}\text { Meta-analysis of } \\
\text { RCTs }\end{array}$ & $\begin{array}{l}\text { Various: inter- } \\
\text { net or comput- } \\
\text { er based }\end{array}$ & $\begin{array}{l}\text { Abstinence and } \\
\text { biochemical mark- } \\
\text { ers }\end{array}$ & $\begin{array}{l}>3 \\
\text { months }\end{array}$ & $\begin{array}{l}\text { Intervention groups had a signif- } \\
\text { icant effect on smoking cessation } \\
\text { (RR } 1.44 ; 95 \% \text { CI } 1.27 \text { to } 1.64 \text { ). } \\
\text { Similar findings were observed } \\
\text { in web-based interventions (RR } \\
1.40 ; 95 \% \text { CI } 1.13 \text { to } 1.72 \text { ) and } \\
\text { in computer-based interventions } \\
\text { (RR } 1.48 ; 95 \% \text { CI } 1.25 \text { to } 1.76 \text { ). }\end{array}$ & $\begin{array}{l}\text { Critically } \\
\text { low }\end{array}$ \\
\hline $\begin{array}{l}\text { Naslund et al } \\
\text { (2017) [93] } \\
\text { (smoking on- } \\
\text { ly) }\end{array}$ & $7(7)$ & $\begin{array}{l}\text { Narrative synthesis } \\
\text { of all study types }\end{array}$ & Social media & Cessation & $\begin{array}{l}\text { 30-365 } \\
\text { days }\end{array}$ & $\begin{array}{l}71 \%(5 / 7) \text { of studies reported } \\
\text { significant effects on smoking- } \\
\text { related outcomes such as greater } \\
\text { abstinence, reduction in relapse, } \\
\text { and an increase in quit attempts. }\end{array}$ & $\begin{array}{l}\text { Critically } \\
\text { low }\end{array}$ \\
\hline $\begin{array}{l}\text { Elaheebocus } \\
\text { et al (2018) } \\
{[56]}\end{array}$ & 7 (134) & $\begin{array}{l}\text { Narrative synthesis } \\
\text { of RCTs }\end{array}$ & Social media & Cessation & $\begin{array}{l}6-48 \\
\text { months }\end{array}$ & $\begin{array}{l}100 \%(7 / 7) \text { of studies on smok- } \\
\text { ing cessation using web-based } \\
\text { social networks had positive re- } \\
\text { sults. }\end{array}$ & $\begin{array}{l}\text { Critically } \\
\text { low }\end{array}$ \\
\hline $\begin{array}{l}\text { Rooke et al } \\
(2010)[112]\end{array}$ & $13(34)$ & $\begin{array}{l}\text { Meta-analysis of } \\
\text { RCTs }\end{array}$ & $\begin{array}{l}\text { Computer } \\
\text { based }\end{array}$ & $\begin{array}{l}\text { Abstinence and re- } \\
\text { duction }\end{array}$ & $\begin{array}{l}1-156 \\
\text { weeks }\end{array}$ & $\begin{array}{l}\text { The weighted average effect size } \\
(\text { Cohen } d \text { ) was } 0.14 ; P<.001 \text { for } \\
\text { studies addressing tobacco use. }\end{array}$ & $\begin{array}{l}\text { Critically } \\
\text { low }\end{array}$ \\
\hline \multicolumn{8}{|l|}{ Nonactive controls } \\
\hline $\begin{array}{l}\text { Chen et al } \\
(2012)[84] \\
\text { (smoking on- } \\
\text { ly) }\end{array}$ & $60(60)$ & $\begin{array}{l}\text { Meta-analysis of } \\
\text { RCTs and quasi- } \\
\text { RCTs }\end{array}$ & $\begin{array}{l}\text { Various: com- } \\
\text { puter and oth- } \\
\text { er electronic } \\
\text { aids }\end{array}$ & Cessation & $\begin{array}{l}2 \text { days- } \\
30 \\
\text { months }\end{array}$ & $\begin{array}{l}\text { Computer and other electronic } \\
\text { aids increase the likelihood of } \\
\text { cessation compared with no inter- } \\
\text { vention or generic self-help mate- } \\
\text { rials but the effect is small (pro- } \\
\text { longed abstinence: RR } 1.32 ; 95 \% \\
\text { CI } 1.21 \text { to } 1.45 \text { ). }\end{array}$ & Low \\
\hline $\begin{array}{l}\text { Krebs et al } \\
(2010)[109]\end{array}$ & $32(88)$ & $\begin{array}{l}\text { Meta-analysis of } \\
\text { RCTs }\end{array}$ & $\begin{array}{l}\text { Computer tai- } \\
\text { lored }\end{array}$ & Abstinence & $\begin{array}{l}24 \\
\text { hours-9 } \\
\text { months }\end{array}$ & $\begin{array}{l}\text { Mean effect for the } 32 \text { studies } \\
\text { reporting point prevalence out- } \\
\text { come was } g=0.16 \text { ( } 95 \% \text { CI } 0.12 \\
\text { to } 0.19) \text {; mean effect for the } 16 \\
\text { studies reporting prolonged absti- } \\
\text { nence measures was } g=0.24 \\
(95 \% \text { CI } 0.20 \text { to } 0.31) \text {. }\end{array}$ & Low \\
\hline
\end{tabular}




\begin{tabular}{|c|c|c|c|c|c|c|c|}
\hline Review & $\begin{array}{l}\text { Relevant } \\
\text { studies, } \mathrm{n} \\
\text { (total } \\
\text { studies) }\end{array}$ & $\begin{array}{l}\text { Method of synthe- } \\
\text { sis }\end{array}$ & Interventions & Outcomes & $\begin{array}{l}\text { Follow- } \\
\text { up }\end{array}$ & Summary of findings & $\begin{array}{l}\text { AMSTAR- } \\
2 \text { rating }\end{array}$ \\
\hline $\begin{array}{l}\text { Covolo et al } \\
(2017)[104]\end{array}$ & $2(40)$ & $\begin{array}{l}\text { Narrative synthesis } \\
\text { of RCTs }\end{array}$ & Mobile apps & $\begin{array}{l}\text { 30-day point } \\
\text { prevalence cessa- } \\
\text { tion }\end{array}$ & 30 days & $\begin{array}{l}1 \text { trial compared } 2 \text { apps and } \\
\text { found no evidence of any differ- } \\
\text { ence; the other found text messag- } \\
\text { ing produced more abstinence } \\
\text { than an app }(P<.05) \text {. }\end{array}$ & $\begin{array}{l}\text { Critically } \\
\text { low }\end{array}$ \\
\hline
\end{tabular}

${ }^{\mathrm{a}} \mathrm{RCT}$ : randomized controlled trial.

${ }^{\mathrm{b}} \mathrm{OR}$ : odds ratio.

${ }^{\mathrm{c}} \mathrm{RR}$ : risk ratio.

${ }^{\mathrm{d}}$ In total, 12 relevant studies were included in the meta-analysis.

Internet interventions were generally found to be effective (Table 6). A total of 3 relevant meta-analyses found small or small-to-medium effects [85,89,91]: pooled RR 1.39 [85]; RR 1.40 [92], and RR 1.43 [88] and a short-term ( $\leq 6$ months) OR 1.29 (95\% CI 1.12 to $1.50 ; P=.001$ ) [91]; the latter showed that internet interventions were also successful for the individual outcomes of prolonged abstinence, that is, not smoking since a quit date (OR 1.43) and 30-day point prevalence abstinence, that is, not smoking one or more days before the follow-up (OR 1.75) [91]. However, one narrative synthesis noted that although the 3 studies that measured smoking cessation all showed significant intervention effects, they were all assessed to be of low quality [100].

The 3 reviews of mobile interventions that focused on SMS text messaging found it to be effective, again with small effects: Cohen $d=0.14$ [94]; OR 1.36 [96]; and OR 1.36 [98]. A fourth meta-analysis differentiated between specific outcomes and found a medium effect (RR 2.19) on biochemically verified continuous abstinence, and a small-to-medium effect (RR 1.51) on verified 7-day point prevalence of smoking cessation was pooled [111]. However, 2 reviews (including 1 meta-analysis) with information on mobile interventions in general did not find that they were effective $[89,99]$.

A meta-analysis of computer-delivered interventions also found a small but significant effect size associated with studies addressing tobacco use (Cohen $d=0.14$ ) [112].

There is mixed evidence from reviews that cover a variety of digital technologies. One narrative synthesis [99] and one meta-analysis found that they were effective, with interventions increasing the odds of smoking cessation at 1 month (OR 1.70) [83]. However, another narrative synthesis that included both phone and internet interventions found that there were mixed results [86].

A total of 2 reviews of social media interventions with narrative syntheses both found favorable effects of the interventions on outcomes related to smoking cessation [56,93].

As seen above, separate reviews of internet, mobile, and computer-delivered interventions found small effects for each. One meta-analysis looked for differences in effect sizes between different modes of intervention and found no statistically significant evidence of any differences in effect sizes of internet interventions, intensive advice, telephone support, individual counseling, or group behavior therapy [89].

\section{Effectiveness of Digital Interventions on Smoking Compared With Nonactive Controls}

There were small effects of digital interventions compared with nonactive controls for all modes of delivery (Table 6). Meta-analyses found small effects for pooled modes of digital interventions compared with controls: 1 month OR $1.70(\mathrm{k}=13)$ [83]; pooled estimate for prolonged abstinence, RR $1.32(\mathrm{k}=60)$ [84], and pooled estimate for point prevalence abstinence, RR 1.14 [84]. Internet interventions were more effective than nonactive controls [88-90,92,97], with 3 meta-analyses finding small effects compared with nonactive controls: RR 1.15 [97], RR 1.46 [89], and RR 1.60 [88]. Computer-tailored interventions had effects sizes of $g=0.16$ for point prevalence outcomes and $g=0.24$ for prolonged abstinence [109]. Mobile phone-based interventions appeared to have similar effectiveness to minimal controls (RR 1.18) and were more effective than no intervention (typically either waiting list control or no further contact until follow-up) [89].

\section{Effectiveness of Digital Interventions on Smoking Compared With Active Controls}

Most reviews concluded that internet interventions were not more effective than active controls, with 3 reviews (including 2 meta-analyses) not finding differences [88,90,97]. This included a meta-analysis that found no significant effects of internet interventions compared with face-to-face counseling or telephone counseling [88]. However, one narrative synthesis was more positive about the greater effect of internet interventions compared with conventional ones [99].

There was no information on mobile interventions compared with active controls, but one meta-analysis with mixed controls noted that the summary effect sizes favored the treatment groups even when 18 of the $20(90 \%)$ controlled trials used an active control and $12(66 \%)$ of these active controls included some smoking-related content, including smoke-free websites, self-help guidebooks, and smartphone apps [94].

\section{Sustainability of Effects on Smoking at Follow-Up}

There was agreement that the effects of internet interventions were sustained for up to 6 months $[83,87,91,95]$. This was quantified as interventions increasing the 6-month abstinence by $17 \%$ [95], increasing the odds of cessation at the 6-month 
follow-up (OR 1.29) [83], and increasing the likelihood of prolonged abstinence (ie, not smoking since a quit date; OR 1.19) [91]. However, there was disagreement about whether there were significant effects at 12-month follow-ups, with 2 reviews in favor [87,89], one quantifying the quit rate at 12 months as $8 \%$ [89] but another reporting that the positive results of internet interventions were not generally maintained at 1-year follow-up assessments [103].

There is mixed evidence on the sustainability of the effects of mobile interventions. A total of 2 meta-analyses found that mobile interventions were effective in the medium term, with a quit rate of $13 \%$ [89] and effects on biochemical measures of quitting at 6 months (RR 1.83) [98]. However, a study of SMS text messages suggested that any effect was not sustained [96].

Regarding computer-delivered interventions, one meta-analysis found that computer programs increased the odds of cessation at the 3-month (OR 2.04), 12-month (OR 1.68), and 18-month follow-up (OR 1.83) [83]. A meta-analysis of computer-delivered interventions did not find evidence of differential effects depending on the length of follow-up or number of sessions [112].

A total of 2 reviews that considered a range of different technologies found evidence of sustained effects. A narrative synthesis of internet and mobile interventions found that the OR for 7-day abstinence at 6 months ranged from 1.6 to 2.7 [99]. A meta-analysis found that digital interventions increased the odds of smoking cessation at 3 months (OR 1.30), 6 months (OR 1.29), and 18 months (OR 1.83) postintervention [83].

In a pooled analysis of web-based and computer-based interventions, the smoking cessation rate was $14.8 \%$ in the intervention group and $14.3 \%$ in the control group at the short-term 3-month follow-up $(P=.42), 11.7 \%$ and $7.0 \%$ at the midterm 6- to 10-month follow-up ( $P<.001$ ), and $9.9 \%$ and
$5.7 \%$ at the long-term 12-month follow-up $(P<.001)$, respectively [92].

One meta-analysis that covered multiple intervention types found that SMS text messaging had the highest OR (2.81) at the 1-month follow-up, followed by the use of computer programs at the 3-month follow-up (OR 2.04), 12-month follow-up (OR 1.68), and 18-month follow-up (OR 1.83) and websites at 6 months (OR 1.37), while a DVD intervention and integrated videotelephony did not increase the odds of cessation compared with no intervention [83].

\section{Alcohol}

A total of 13 papers covered alcohol [73,77-82,99,103,104,111-113]. Multimedia Appendix 5 presents a breakdown of the study characteristics.

\section{Effectiveness of Digital Interventions on Alcohol Consumption Compared With Mixed (Active and Nonactive) Controls}

In total, 7 of $8(88 \%)$ reviews with mixed controls reported favorable evidence that digital interventions were effective in decreasing alcohol consumption (Table 7), although one review noted that the controls were just as effective [103]. A total of 2 meta-analyses reported small effect sizes (Cohen $d=0.26$ [112] and Cohen $d=0.14$ [113]). A narrative synthesis of interventions using novel technologies found that, in studies finding benefits and reporting compliance with drinking recommendation as an outcome, the OR for drinking within the recommended limit ranged from 1.7 to 3.7 (small/medium to medium/large effects) [99]. In contrast, the sixth review, which surveyed mobile phone interventions, reported that the results were inconclusive for alcohol reduction, and the authors declined to perform a meta-analysis because the results were self-reported and therefore at risk of overstating the benefits [111]. 
Table 7. Effectiveness of digital interventions on alcohol consumption, sorted by controls and further ordered by intervention type.

\begin{tabular}{|c|c|c|c|c|c|c|c|}
\hline Review & $\begin{array}{l}\text { Relevant } \\
\text { studies, } \mathrm{n} \\
\text { (total } \\
\text { studies) }\end{array}$ & $\begin{array}{l}\text { Method of synthe- } \\
\text { sis }\end{array}$ & Interventions & Outcomes & $\begin{array}{l}\text { Follow- } \\
\text { up }\end{array}$ & Summary of findings & $\begin{array}{l}\text { AMSTAR- } \\
2 \text { rating }\end{array}$ \\
\hline \multicolumn{8}{|c|}{ Mixed (active and nonactive) controls } \\
\hline $\begin{array}{l}\text { Afshin et } \\
\text { al }(2016) \\
{[99]}\end{array}$ & $47(224)$ & $\begin{array}{l}\text { Narrative synthesis } \\
\text { of RCTs }{ }^{\mathrm{a}} \text { and } \\
\text { quasiexperimental } \\
\text { studies }\end{array}$ & $\begin{array}{l}\text { Various: inter- } \\
\text { net, mobile, } \\
\text { computer soft- } \\
\text { ware, and sen- } \\
\text { sors }\end{array}$ & $\begin{array}{l}\text { Alcohol frequency } \\
\text { and quantity, binge } \\
\text { drinking, estimated } \\
\text { blood alcohol concen- } \\
\text { tration, alcohol depen- } \\
\text { dency, and Alcohol } \\
\text { Use Disorder Identifi- } \\
\text { cation Test scores }\end{array}$ & $\begin{array}{l}1 \text { week- } \\
2 \text { years }\end{array}$ & $\begin{array}{l}34 \%(39 / 47) \text { of studies, } 41 \text { RCTs } \\
\text { and } 6 \text { quasiexperimental, report- } \\
\text { ed a significant decrease in alco- } \\
\text { hol use; } 83 \% \text { (33/41) of RCTs } \\
\text { reported statistically significant } \\
\text { benefits. }\end{array}$ & $\begin{array}{l}\text { Critically } \\
\text { low }\end{array}$ \\
\hline $\begin{array}{l}\text { Chebli et } \\
\text { al }(2016) \\
{[103]}\end{array}$ & $2(16)$ & $\begin{array}{l}\text { Narrative synthesis } \\
\text { of RCTs }\end{array}$ & Internet & $\begin{array}{l}\text { Cessation and reduc- } \\
\text { tion of alcohol }\end{array}$ & $\begin{array}{l}1-12 \\
\text { months }\end{array}$ & $\begin{array}{l}\text { Both studies demonstrated posi- } \\
\text { tive treatment outcomes in both } \\
\text { arms, but there were no differ- } \\
\text { ences between internet interven- } \\
\text { tion and control. }\end{array}$ & $\begin{array}{l}\text { Critically } \\
\text { low }\end{array}$ \\
\hline $\begin{array}{l}\text { Kaner et } \\
\text { al }(2017) \\
{[77](\mathrm{Al}-} \\
\text { cohol on- } \\
\text { ly) }\end{array}$ & $57(57)$ & $\begin{array}{l}\text { Narrative synthesis } \\
\text { and meta-analysis } \\
\text { of RCTs }\end{array}$ & $\begin{array}{l}\text { Computer and } \\
\text { mobile }\end{array}$ & $\begin{array}{l}\text { Alcohol consumption } \\
\text { and frequency }\end{array}$ & $\begin{array}{l}1-12 \\
\text { months }\end{array}$ & $\begin{array}{l}\text { Alcohol consumption reduced by } \\
\text { approximately } 23 \text { g per week } \\
\text { ( } 95 \% \text { CI } 15 \text { to 30) at follow-up } \\
\text { (1-12 months; based on } 41 \text { stud- } \\
\text { ies). Frequency of consumption } \\
\text { reduced (based on } 15 \text { studies): } \\
\text { participants who engaged with } \\
\text { digital interventions had less than } \\
\text { one drinking day per month few- } \\
\text { er than no intervention controls } \\
\text { (moderate - quality evidence); } \\
\text { had about one binge drinking } \\
\text { session less per month in the in- } \\
\text { tervention group (moderate - } \\
\text { quality evidence); and drank one } \\
\text { unit per occasion less than no in- } \\
\text { tervention control participants } \\
\text { (moderate - quality evidence). } \\
\text { Compared with face-to-face inter- } \\
\text { ventions, there was no difference } \\
\text { in alcohol consumption at the } \\
\text { end of follow-up (mean differ- } \\
\text { ence } 0.52 \text { g/week; } 95 \% \text { CI } \\
-24.59 \text { to } 25.63 \text {; low - quality } \\
\text { evidence). }\end{array}$ & Low \\
\hline $\begin{array}{l}\text { Kolar et } \\
\text { al }(2015) \\
{[78](\mathrm{Al}-} \\
\text { cohol on- } \\
\text { ly) }\end{array}$ & $2(18)$ & $\begin{array}{l}\text { Narrative synthesis } \\
\text { of all studies }\end{array}$ & Internet & $\begin{array}{l}\text { Alcohol quantity and } \\
\text { frequency }\end{array}$ & 1 month & $\begin{array}{l}100 \%(2 / 2) \text { of studies found re- } \\
\text { duced alcohol consumption in } \\
\text { both arms but no significant dif- } \\
\text { ferences between arms. }\end{array}$ & $\begin{array}{l}\text { Critically } \\
\text { low }\end{array}$ \\
\hline $\begin{array}{l}\text { Palmer et } \\
\text { al }(2018) \\
{[111]}\end{array}$ & $8(71)$ & $\begin{array}{l}\text { Narrative synthesis } \\
\text { of RCTs }\end{array}$ & Mobile & $\begin{array}{l}\text { Self-report alcohol } \\
\text { consumption }\end{array}$ & $\begin{array}{l}\text { Not re- } \\
\text { ported }\end{array}$ & $\begin{array}{l}\text { The effects of alcohol reduction } \\
\text { interventions were inconclusive. }\end{array}$ & Moderate \\
\hline $\begin{array}{l}\text { Rooke et } \\
\text { al }(2010) \\
{[112]}\end{array}$ & $9(34)$ & $\begin{array}{l}\text { Meta-analysis of } \\
\text { RCTs }\end{array}$ & $\begin{array}{l}\text { Computer-deliv- } \\
\text { ered }\end{array}$ & $\begin{array}{l}\text { Abstinence and reduc- } \\
\text { tion of alcohol }\end{array}$ & $\begin{array}{l}1-156 \\
\text { weeks }\end{array}$ & $\begin{array}{l}\text { The weighted average effect size } \\
(\text { Cohen } d) \text { was } 0.20(P<.001) \text {. }\end{array}$ & $\begin{array}{l}\text { Critically } \\
\text { low }\end{array}$ \\
\hline $\begin{array}{l}\text { Vernon et } \\
\text { al }(2010) \\
{[81](\mathrm{Al}-} \\
\text { cohol on- } \\
\text { ly) }\end{array}$ & $15(15)$ & $\begin{array}{l}\text { Narrative synthesis } \\
\text { of all studies }\end{array}$ & $\begin{array}{l}\text { Computer-deliv- } \\
\text { ered }\end{array}$ & Alcohol consumption & $\begin{array}{l}30 \text { days- } \\
12 \\
\text { months }\end{array}$ & $\begin{array}{l}\text { All but one intervention showed } \\
\text { significant improvement in at } \\
\text { least one drinking-related out- } \\
\text { come. However, interventions } \\
\text { were heterogenous and preinter- } \\
\text { vention alcohol consumption was } \\
\text { not standardized. }\end{array}$ & $\begin{array}{l}\text { Critically } \\
\text { low }\end{array}$ \\
\hline
\end{tabular}




\begin{tabular}{|c|c|c|c|c|c|c|c|}
\hline Review & $\begin{array}{l}\text { Relevant } \\
\text { studies, } \mathrm{n} \\
\text { (total } \\
\text { studies) }\end{array}$ & $\begin{array}{l}\text { Method of synthe- } \\
\text { sis }\end{array}$ & Interventions & Outcomes & $\begin{array}{l}\text { Follow- } \\
\text { up }\end{array}$ & Summary of findings & $\begin{array}{l}\text { AMSTAR- } \\
2 \text { rating }\end{array}$ \\
\hline $\begin{array}{l}\text { Webb et } \\
\text { al }(2010) \\
{[113]}\end{array}$ & $9(85)$ & $\begin{array}{l}\text { Meta-analysis of } \\
\text { RCTs }\end{array}$ & Internet & Alcohol consumption & $\begin{array}{l}\text { Not re- } \\
\text { ported }\end{array}$ & $\begin{array}{l}\text { Small effects were observed for } \\
\text { alcohol consumption (Cohen } \\
d_{+}=0.14 ; \mathrm{k}=9 ; 95 \% \text { CI } 0.00 \text { to } \\
0.27 \text { ). }\end{array}$ & $\begin{array}{l}\text { Critically } \\
\text { low }\end{array}$ \\
\hline
\end{tabular}

\section{Nonactive controls}

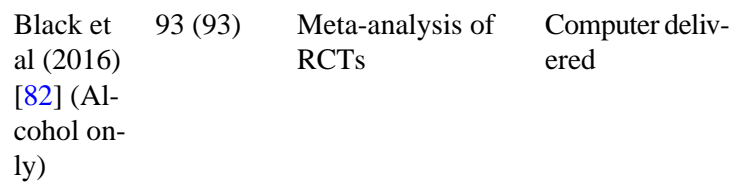

Alcohol consumption: Up to 2 Small effects averaging across total consumption $\quad$ years timepoints, Cohen $d=0.007$

Critically over a period of time; average alcohol consumption per drinking occasion or drinking day; peak consumption-max consumed on one occasion. Frequency of heavy episodic drinking and of any alcohol consumption

$\begin{aligned} & \text { Covolo et } \\ & \text { al }(2017)\end{aligned}$
$[104]$

Narrative synthesis Mobile

Alcohol frequency

(heavy episodic drinking frequency) to Cohen $d=0.15$ (total consumption); in the short term, there were small-to-medium effects (Cohen $d+=0.16$ to 0.31 ) and significant effects on all outcomes except drinking frequency; in the medium-to-long term, they produced small (Cohen $d+=0.07$ to 0.12 ), significant effects on all outcomes.

Contrary to expectation, it was ign the mobile app signif- low icantly increased the frequency of drinking occasions compared with the control group $(P=.001)$.

$\begin{array}{llll}\begin{array}{l}\text { Riper et } \\ \text { al (2011) }\end{array} & 9(9) & \begin{array}{l}\text { Meta-analysis of } \\ \text { RCTs }\end{array} & \text { Internet } \\ \text { [79] (Al- } & & & \\ \text { cohol on- } & & & \\ \text { ly) } & & & \\ \text { Riper et } & 16(16) & \text { Meta-analysis of } & \text { Internet } \\ \text { al (2014) } & & \text { RCTs } & \\ \text { [80] (Al- } & & & \\ \text { cohol on- } & & & \\ \text { ly) } & & \end{array}$

\section{Alcohol consumption} Up to 12 months

Alcohol consumption

1 to 12 months

An overall medium effect size ( $g=0.44 ; 95 \%$ CI 0.17 to 0.71 ; random effect model) in favor of the intervention groups was found.

A small but significant overall effect size in favor of internet interventions $(g=0.20 ; 95 \%$ CI 0.13 to $0.27 ; P=.001$ ) was found. Participants in internet-based interventions consumed approximately $22 \mathrm{~g}$ of ethanol less and were more likely to adhere to low-risk drinking guidelines (risk difference $0.13 ; 95 \%$ CI 0.09 to $0.17 ; P=.001)$.

Tsoli et al 4 (15) (2018) Meta-analysis of
RCTs

Interactive voice responses

Alcohol consumption 6 weeks12 months

The meta-analysis of included studies demonstrated that interac- low tive voice response-based inter-

[73] nificant had no statistically sigsumption $(g=-0.077 ; 95 \%$ CI -0.162 to $0.007 ; \mathrm{k}=4 ; P=.07)$.

Critically low

Critically low

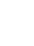
.

${ }^{\mathrm{a}} \mathrm{RCT}$ : randomized controlled trial.

\section{Effectiveness of Digital Interventions on Alcohol Consumption Compared With Nonactive Controls}

In total, 4 of $6(67 \%)$ reviews with nonactive controls found an effect of the intervention (Table 7). Of the 4 meta-analyses, 2 found a medium effect on alcohol consumption $(g=0.44$ [80] and $g=0.44$ [79]). A third found a small-to-medium effect on total alcohol consumption (Cohen $d+=0.31$ ), small effects on 2 other consumption measures, and a measure of frequency of heavy drinking episodes (Cohen $d+=0.16-0.19$ ) and no effect on drinking frequency [82]. The fourth review found no effect [73].

Two studies quantified the effect on alcohol consumption, finding a reduction in weekly consumption of $22 \mathrm{~g}$ of alcohol [80] and $23 \mathrm{~g}$ of alcohol [77], approximately 3 UK units. However, when studies with a high risk of bias were excluded, this number decreased to $11 \mathrm{~g}$ of alcohol (or $1.5 \mathrm{UK}$ units) per week [77]. With regard to frequency of consumption, participants who were given digital interventions had less than one drinking day per month, which is fewer compared with 
no-intervention controls (moderate-quality evidence); had approximately 1 binge-drinking session less per month in the intervention group compared with no-intervention controls (moderate-quality evidence); and drank 1 unit per occasion, which is less than the no-intervention control participants (moderate-quality evidence) [77]. Participants in internet interventions were significantly more likely to adhere to low-risk drinking guidelines at the immediate posttreatment follow-up, compared with the no-intervention controls [80].

Only one review was negative. A narrative synthesis of mobile apps reported that there was a single RCT on alcohol reduction where, contrary to expectation, the frequency of drinking occasions was higher in the intervention group [104].

\section{Effectiveness of Digital Interventions on Alcohol Consumption Compared With Active Controls}

In total, 2 reviews that separated active controls found that there were no significant differences between the intervention and control groups [77,99], one of which was specifically compared with face-to-face controls (Table 7) [77].

\section{Sustainability of Effects on Alcohol Consumption at Follow-Up}

The effectiveness of the interventions seemed to decrease over time (Table 7). One meta-analysis, which reported small-to-medium effects in the short term (Cohen $d+=0.16-0.19)$, found that in the medium to long term, there were small (Cohen $d+=0.07-0.12$ ), significant effects on all outcomes [82]. A total of 2 reviews reported that, for internet-based intervention studies where there were 3-, 6-, or 12-month follow-up data, no significant differences in effect remained in later follow-up $[80,103]$. In contrast, one review of computer- or mobile-delivered interventions found that positive differences in measures of drinking were seen at 1, 6, and 12 months [77], and one review of internet interventions found a medium effect size $(g=0.39)$, lasting up to 6 or 9 months posttreatment, as compared with no intervention; the effects of the interventions beyond 9 months could not be assessed, but 2 studies in the review suggested that they had faded out by 12 months [79].

\section{Other Combinations}

\section{Review Characteristics}

A total of 11 reviews covered a number of areas of behavior [101-107,109,110,112,113]. The breakdown of their characteristics is shown in Multimedia Appendix 5.

\section{Effectiveness of Other Digital Combination Interventions Compared With Mixed (Active and Nonactive) Controls}

All 5 reviews of internet interventions concluded that they were effective in changing behavior (Table 8). A total of 3 meta-analyses found small effects: Cohen $d=0.19$ [105], Cohen $d=0.139$ [110], and Cohen $d=0.16$ [113]. However, one study reported that the effect sizes were heterogeneous [113]. The fourth meta-analysis quantified the effects on health outcomes, finding statistically significant reductions in systolic blood pressure (mean difference $-2.66 \mathrm{~mm} \mathrm{Hg}$ ), diastolic blood pressure (mean difference $-1.26 \mathrm{~mm} \mathrm{Hg}$ ), glycated hemoglobin level (mean difference $-0.13 \%$ ), and low-density lipoprotein cholesterol level (mean difference -2.18 mg/dL) [102]. 
Table 8. Effectiveness of digital interventions on other combinations of outcomes, sorted by controls and further sorted by intervention type.

\begin{tabular}{|c|c|c|c|c|c|c|c|}
\hline Review & $\begin{array}{l}\text { Relevant } \\
\text { studies, n } \\
\text { (total stud- } \\
\text { ies) }\end{array}$ & $\begin{array}{l}\text { Method of synthe- } \\
\text { sis }\end{array}$ & Interventions & Outcomes & Follow-up & Summary of findings & $\begin{array}{l}\text { AMSTAR-2 } \\
\text { rating }\end{array}$ \\
\hline \multicolumn{8}{|c|}{ Mixed (active and nonactive) controls } \\
\hline $\begin{array}{l}\text { Beishuizen } \\
\text { et al (2016) } \\
{[102]}\end{array}$ & $15(57)$ & $\begin{array}{l}\text { Meta-analysis of } \\
\text { RCTs }^{\mathrm{a}}\end{array}$ & Internet & $\begin{array}{l}\text { Systolic blood } \\
\text { pressure, dias- } \\
\text { tolic blood } \\
\text { pressure, } \\
\mathrm{HbA}_{1 \mathrm{c}} \text { b level, } \\
\text { cholesterol } \\
\text { level, weight, } \\
\text { and level of } \\
\text { physical activ- } \\
\text { ity }\end{array}$ & $\begin{array}{l}3-60 \\
\text { months }\end{array}$ & $\begin{array}{l}\text { Intervention groups had a reduction } \\
\text { in systolic blood pressure (mean } \\
\text { difference }-2.66 \mathrm{~mm} \mathrm{Hg} \text {; } 95 \% \mathrm{CI} \\
-3.81 \text { to }-1.52 \text { ), diastolic blood } \\
\text { pressure (mean difference }-1.26 \mathrm{~mm} \\
\mathrm{Hg} ; 95 \% \mathrm{CI}-1.92 \text { to }-0.60), \mathrm{HbA}_{1 \mathrm{c}} \\
\text { level (mean difference }-0.13 \% ; 95 \% \\
\mathrm{CI}-0.22 \text { to }-0.05 \text { ), and } \mathrm{LDL}^{\mathrm{c}} \\
\text { cholesterol level (mean difference } \\
-2.18 \mathrm{mg} / \mathrm{dL} ; 95 \% \mathrm{CI}-3.96 \text { to } \\
-0.41 \text { ). There were larger effects in } \\
\text { internet interventions that combined } \\
\text { the internet application with human } \\
\text { support (blended care). }\end{array}$ & Low \\
\hline $\begin{array}{l}\text { Chebli et al } \\
(2016) \\
{[103]}\end{array}$ & $11(16)$ & $\begin{array}{l}\text { Narrative synthesis } \\
\text { of RCTs }\end{array}$ & Internet & $\begin{array}{l}\text { Cessation and } \\
\text { reduction (for } \\
\text { both alcohol } \\
\text { and smoking) }\end{array}$ & $\begin{array}{l}1-12 \\
\text { months }\end{array}$ & $\begin{array}{l}\text { Internet-based interventions may } \\
\text { have a positive effect on smoking } \\
\text { cessation. Several studies found that } \\
\text { web-based use and number of log- } \\
\text { ins were positively associated with } \\
\text { quit outcomes. Both studies on alco- } \\
\text { hol demonstrated positive treatment } \\
\text { outcomes in both arms, but there } \\
\text { were no differences between the in- } \\
\text { ternet-based intervention and con- } \\
\text { trol. }\end{array}$ & $\begin{array}{l}\text { Critically } \\
\text { low }\end{array}$ \\
\hline $\begin{array}{l}\text { Webb et al } \\
(2010) \\
{[113]}\end{array}$ & $85(85)$ & $\begin{array}{l}\text { Meta-analysis of } \\
\text { RCTs }\end{array}$ & Internet & $\begin{array}{l}\text { Smoking absti- } \\
\text { nence, level of } \\
\text { physical activ- } \\
\text { ity, alcohol } \\
\text { consumption, } \\
\text { and dietary be- } \\
\text { havior }\end{array}$ & $\begin{array}{l}3-12 \\
\text { months }\end{array}$ & $\begin{array}{l}\text { Interventions had a statistically } \\
\text { small but significant effect on } \\
\text { health-related behavior (Cohen } \\
d+=0.16 ; 95 \% \text { CI } 0.09 \text { to } 0.23 \text { ). The } \\
\text { effect size of interventions targeting } \\
\text { a single area of health behavior was } \\
\text { not significantly different to the ef- } \\
\text { fect size of those targeting multiple } \\
\text { areas of health behaviors, but the } \\
\text { numerical difference was in favor } \\
\text { of single-area studies (Cohen } \\
d+=0.17 \text { versus Cohen } d+=0.12) \text {. }\end{array}$ & $\begin{array}{l}\text { Critically } \\
\text { low }\end{array}$ \\
\hline
\end{tabular}




\begin{tabular}{|c|c|c|c|c|c|c|c|}
\hline Review & $\begin{array}{l}\text { Relevant } \\
\text { studies, n } \\
\text { (total stud- } \\
\text { ies) }\end{array}$ & $\begin{array}{l}\text { Method of synthe- } \\
\text { sis }\end{array}$ & Interventions & Outcomes & Follow-up & Summary of findings & $\begin{array}{l}\text { AMSTAR-2 } \\
\text { rating }\end{array}$ \\
\hline $\begin{array}{l}\text { Lustria et } \\
\text { al }(2013) \\
{[110]}\end{array}$ & $40(40)$ & $\begin{array}{l}\text { Meta-analysis of } \\
\text { experimental and } \\
\text { quasiexperimental } \\
\text { studies }\end{array}$ & $\begin{array}{l}\text { Tailored in- } \\
\text { ternet-based } \\
\text { interventions }\end{array}$ & $\begin{array}{l}\text { Levels of } \\
\text { physical activ- } \\
\text { ity, fruit and } \\
\text { vegetable in- } \\
\text { take, saturated } \\
\text { fat intake, and } \\
\text { abstinence } \\
\text { from smoking } \\
\text { and alcohol } \\
\text { consumption }\end{array}$ & $\begin{array}{l}1-24 \\
\text { months }\end{array}$ & 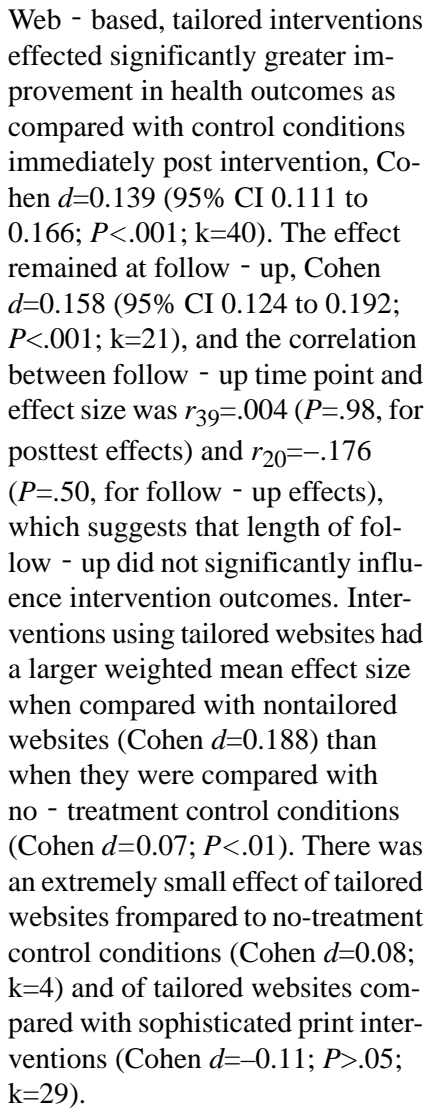 & $\begin{array}{l}\text { Critically } \\
\text { low }\end{array}$ \\
\hline $\begin{array}{l}\text { Covolo et } \\
\text { al }(2017) \\
{[104]}\end{array}$ & $39(40)$ & $\begin{array}{l}\text { Narrative synthesis } \\
\text { of RCTs }\end{array}$ & Mobile apps & $\begin{array}{l}\text { BMI and waist } \\
\text { circumfer- } \\
\text { ence; various } \\
\text { physical activ- } \\
\text { ity levels; fruit } \\
\text { and vegetable } \\
\text { intake, high- } \\
\text { sugar food in- } \\
\text { take, smoking } \\
\text { cessation, and } \\
\text { number of } \\
\text { drinking days }\end{array}$ & $\begin{array}{l}6 \text { months- } \\
2 \text { years }\end{array}$ & $\begin{array}{l}\text { Only } 25 \%(10 / 40) \text { of RCTs found } \\
\text { statistical differences between inter- } \\
\text { vention and control groups. }\end{array}$ & $\begin{array}{l}\text { Critically } \\
\text { low }\end{array}$ \\
\hline $\begin{array}{l}\text { Armanasco } \\
\text { et al (2017) } \\
{[101]}\end{array}$ & $35(51)$ & $\begin{array}{l}\text { Meta-analysis of } \\
\text { RCTs and quasiex- } \\
\text { perimental studies }\end{array}$ & $\begin{array}{l}\text { Text messag- } \\
\text { ing }\end{array}$ & $\begin{array}{l}\text { Weight, level } \\
\text { of physical ac- } \\
\text { tivity, and } \\
\text { smoking cessa- } \\
\text { tion }\end{array}$ & $\begin{array}{l}1 \text { to } 66 \\
\text { weeks }\end{array}$ & $\begin{array}{l}\text { The overall pooled effect of interven- } \\
\text { tions was Cohen } d=0.24 \text { ( } 95 \% \mathrm{CI} \\
0.16 \text { to } 0.32 ; P<.001 ; \mathrm{k}=35) \text { using } \\
\text { outcome data collected most proxi- } \\
\text { mal to the intervention end. } 7 \text { stud- } \\
\text { ies collected data following a no- } \\
\text { intervention maintenance period and } \\
\text { showed a small but significant } \\
\text { pooled maintenance effect (Cohen } \\
d=0.17 ; 95 \% \text { CI } 0.03 \text { to } 0.31 ; \\
P=.017 ; \mathrm{k}=7) \text {. }\end{array}$ & $\begin{array}{l}\text { Critically } \\
\text { low }\end{array}$ \\
\hline
\end{tabular}




\begin{tabular}{|c|c|c|c|c|c|c|c|}
\hline Review & $\begin{array}{l}\text { Relevant } \\
\text { studies, } \mathrm{n} \\
\text { (total stud- } \\
\text { ies) }\end{array}$ & $\begin{array}{l}\text { Method of synthe- } \\
\text { sis }\end{array}$ & Interventions & Outcomes & Follow-up & Summary of findings & $\begin{array}{l}\text { AMSTAR-2 } \\
\text { rating }\end{array}$ \\
\hline $\begin{array}{l}\text { Head et al } \\
(2013) \\
{[107]}\end{array}$ & $12(19)$ & $\begin{array}{l}\text { Meta-analysis of } \\
\text { RCTs }\end{array}$ & $\begin{array}{l}\text { Text messag- } \\
\text { ing }\end{array}$ & $\begin{array}{l}\text { Smoking ces- } \\
\text { sation, weight } \\
\text { loss, and level } \\
\text { of physical ac- } \\
\text { tivity }\end{array}$ & $\begin{array}{l}\text { Mean } \\
81.26 \text { days }\end{array}$ & $\begin{array}{l}\text { The overall weighted mean effect } \\
\text { size representing the impact of these } \\
\text { interventions on health outcomes } \\
\text { was Cohen } d=0.329 \text { ( } 95 \% \text { CI } 0.274 \\
\text { to } 0.385 ; P<.001) \text {. Correlations be- } \\
\text { tween effect size and follow-up } \\
\left(r_{18}=-.12 ; P=.62\right) \text { and effect size } \\
\text { and retention }\left(r_{18}=.14 ; P=.56\right) \text { were } \\
\text { not statistically significant. Effect } \\
\text { sizes for interventions that em- } \\
\text { ployed no-treatment control groups } \\
\text { (Cohen } d=0.369), \text { however, were } \\
\text { significantly larger than those that } \\
\text { employed alternative comparisons } \\
\text { (Cohen } d=0.226 ; Q_{B}=.5 .16, \mathrm{df}=1 ; \\
P=.02) \text {. }\end{array}$ & $\begin{array}{l}\text { Critically } \\
\text { low }\end{array}$ \\
\hline $\begin{array}{l}\text { De Leon et } \\
\text { al }(2014) \\
{[106]}\end{array}$ & $55(55)$ & $\begin{array}{l}\text { Narrative synthesis } \\
\text { of RCTs }\end{array}$ & $\begin{array}{l}\text { Text mes- } \\
\text { sages and } \\
\text { emails } \\
\text { (prompts) }\end{array}$ & $\begin{array}{l}\text { Smoking ces- } \\
\text { sation, diet in- } \\
\text { take, and } \\
\text { physical activ- } \\
\text { ity level }\end{array}$ & $\begin{array}{l}3 \text { weeks-9 } \\
\text { months }\end{array}$ & $\begin{array}{l}76 \%(42 / 55) \text { of articles found statis- } \\
\text { tically significant positive behav- } \\
\text { ioral outcomes of prompts, the mode } \\
\text { by which the prompt was sent did } \\
\text { not seem to impact its success. }\end{array}$ & $\begin{array}{l}\text { Critically } \\
\text { low }\end{array}$ \\
\hline $\begin{array}{l}\text { Rooke et al } \\
(2010) \\
{[112]}\end{array}$ & $34(34)$ & $\begin{array}{l}\text { Meta-analysis of } \\
\text { RCTs }\end{array}$ & $\begin{array}{l}\text { Computer } \\
\text { delivered }\end{array}$ & $\begin{array}{l}\text { Abstinence } \\
\text { and reduction } \\
\text { for both smok- } \\
\text { ing and alco- } \\
\text { hol }\end{array}$ & $\begin{array}{l}1-156 \\
\text { weeks }\end{array}$ & $\begin{array}{l}\text { The weighted average effect size } \\
\text { (Cohen } d \text { ) was } 0.20(P<.001) \text {; how- } \\
\text { ever, lower effect sizes were associ- } \\
\text { ated with studies addressing tobacco } \\
\text { use (Cohen } d=0.14) \text {. There was sig- } \\
\text { nificant heterogeneity between } \\
\text { studies targeting tobacco versus al- } \\
\text { cohol use ( } Q=5.65 ; P=.02) \text {, with } \\
\text { studies on alcohol consumption } \\
\text { producing significantly higher effect } \\
\text { sizes than tobacco studies (Cohen } \\
d=0.26 \text { and } 0.12 \text {, respectively). Ef- } \\
\text { fect sizes were higher for studies in } \\
\text { which the comparison condition was } \\
\text { an attention/placebo (Cohen } d=0.22) \\
\text { relative to studies in which the } \\
\text { comparison condition was an active } \\
\text { comparison (Cohen } d=0.10) \text {; studies } \\
\text { employing active treatments as the } \\
\text { comparison condition mainly pro- } \\
\text { duced effect sizes close to zero. }\end{array}$ & $\begin{array}{l}\text { Critically } \\
\text { low }\end{array}$ \\
\hline \multicolumn{8}{|c|}{ Nonactive controls } \\
\hline
\end{tabular}




\begin{tabular}{|c|c|c|c|c|c|c|c|}
\hline Review & $\begin{array}{l}\text { Relevant } \\
\text { studies, n } \\
\text { (total stud- } \\
\text { ies) }\end{array}$ & $\begin{array}{l}\text { Method of synthe- } \\
\text { sis }\end{array}$ & Interventions & Outcomes & Follow-up & Summary of findings & $\begin{array}{l}\text { AMSTAR-2 } \\
\text { rating }\end{array}$ \\
\hline $\begin{array}{l}\text { Krebs et al } \\
(2010) \\
{[109]}\end{array}$ & $76(88)$ & $\begin{array}{l}\text { Meta-analysis of } \\
\text { RCTs }\end{array}$ & $\begin{array}{l}\text { Computer- } \\
\text { tailored inter- } \\
\text { ventions }\end{array}$ & $\begin{array}{l}\text { Dietary intake } \\
\text { of fat, vegeta- } \\
\text { bles, fruit; var- } \\
\text { ious levels of } \\
\text { physical activ- } \\
\text { ity, and smok- } \\
\text { ing abstinence } \\
\text { over various } \\
\text { timescales }\end{array}$ & $\begin{array}{l}1-24 \\
\text { months }\end{array}$ & $\begin{array}{l}\text { The overall effect size was } g=0.17 \\
\text { ( } 95 \% \text { CI } 0.14 \text { to } 0.19 \text { ) using a fixed } \\
\text { effects model and } g=0.17 \text { using a } \\
\text { random effects model ( } 95 \% \text { CI } 0.14 \\
\text { to } 0.20 \text { ). Effects peak from } 4 \text { to } 12 \\
\text { months postbaseline with a mean } \\
\text { effect size of } g=0.20 \text {, and while they } \\
\text { decline after } 12 \text { months postbase- } \\
\text { line, the mean effect size at long- } \\
\text { term follow-up ( } g=0.12 \text { ) remains to } \\
\text { be statistically significant ( } 95 \% \text { CI } \\
0.08 \text { to } 0.16 \text { ). The meta-analysis } \\
\text { found a trend for increasing effect } \\
\text { sizes across studies that intervened } \\
\text { on } 1 \text { ( } g=0.15 \text { ), } 2 \text { ( } g=0.21 \text { ), and } 3 \\
\text { ( } g=0.24) \text { areas of behavior, but this } \\
\text { trend did not continue with the } 1 \\
\text { study that intervened on } 4 \text { areas } \\
\text { ( } g=0.12 \text { ). }\end{array}$ & Low \\
\hline
\end{tabular}

${ }^{\mathrm{a}} \mathrm{RCT}$ : randomized controlled trial.

${ }^{b} \mathrm{HbA}_{1 \mathrm{c}}$ : glycated hemoglobin.

${ }^{\mathrm{c}}$ LDL: low-density lipoprotein.

Of the 3 reviews of mobile interventions, a review of SMS text messaging concluded that they were effective, but the narrative synthesis of apps was the only review in the other combinations category that did not find a preponderance of positive results [104]. The effect sizes for the SMS text messaging interventions were small (Cohen $d=0.24$ [101] and Cohen $d=0.329$ [107]), and the effect sizes in the meta-analysis were heterogeneous [107].

Other modes of intervention continued in the pattern of positive, albeit small, effects. Computer-delivered interventions had a small effect (Cohen $d=0.20$ ) [112]. Prompts delivered by SMS text messaging or email were effective in changing diet, physical activity, and smoking behaviors [106].

\section{Relative Effect of Interventions by Number of Behaviors Targeted}

A total of 3 meta-analyses that compared interventions targeting single versus multiple areas of behaviors found that both types of interventions were effective but they differed in whether they found interventions with single or multiple targets to be more effective. A total of 2 meta-analyses of internet-based interventions found that the effect size of interventions targeting a single area of health behavior was not significantly different to the effect size of those targeting multiple areas of health behaviors, but the numerical difference was in favor of single-area studies (Cohen $d=0.146$ vs Cohen $d=0.121$ for multiple-behavior studies [110]; Cohen $d+=0.17$ vs Cohen $d+=0.12$ [113]). However, the computer feedback-based meta-analysis found a trend for increased effect sizes across studies that intervened in $1(g=0.15), 2(g=0.21)$, and $3(g=0.24)$ areas of behavior, but this trend did not continue with the one study that intervened in 4 areas $(g=0.12)$ [109]. The review covered diet, physical activity, smoking cessation, and mammography.

\section{Relative Effect of Intervention by Area of Behaviors Targeted}

The review of SMS text messaging interventions found that interventions targeting smoking cessation and physical activity were more successful than interventions targeting other areas of health behavior, including alcohol and weight loss [107]. In contrast, a review of computer-delivered interventions found that interventions targeting smoking had statistically significantly lower effect sizes than those targeting alcohol (Cohen $d=0.26$ and 0.12 , respectively) [112].

\section{Effect of Other Combination Interventions Compared With Nonactive Controls}

Digital interventions had their largest effect sizes when compared with nonactive controls; however, the effect sizes were generally small. Internet interventions had the largest effect size when compared with waitlists and placebos, but the effect sizes were small: Cohen $d=0.28$ [105] and Cohen $d=0.22$ [107]. Tailored websites had extremely small effect sizes when compared with no-treatment control conditions (Cohen $d=0.07$ ), and the effects were not statistically significant compared with nontailored print materials [110]. There were medium effect sizes for SMS text messaging interventions that employed no-treatment control groups (Cohen $d=0.369$ ) [107].

\section{Effect of Other Combination Interventions Compared With Active Controls}

The effects of digital interventions compared with active controls were very small or nonexistent.

A total of 2 meta-analyses found that there was an extremely small effect size (Cohen $d=0.08$ ) [110] or no significant difference [105] when internet-based interventions were compared with sophisticated print interventions. There were larger effects in internet-based interventions that combined 
internet application with human support (blended care) [102]. Computer-delivered interventions had very small effect sizes when the comparison condition was an active comparison (Cohen $d=0.10$ ); studies employing active treatments as the comparison condition mainly produced effect sizes close to zero [112]. SMS text messaging interventions had small effect sizes compared with active comparisons (Cohen $d=0.226$ ), which was statistically significantly smaller than their effect size compared with the no-treatment control groups [107].

Although digital feedback was effective, it seems that it was no more effective than feedback delivered by nondigital means, and 2 reviews that examined feedback interventions found that the medium did not affect behavior change (SMS text messaging, print, email, telephone, newspaper articles [106] and print, computer, telephone, etc [109]).

\section{Sustainability of Effects at Follow-Up}

There was mixed evidence on the sustainability of interventions. A meta-analysis of internet-based interventions found the largest effect size at 1 month to 4 months [105], but a meta-analysis of computer-tailored interventions found that effects peaked from 4 months to 12 months postbaseline [109]. A total of 3 meta-analyses found that the correlation between effect size and follow-up was not significant for internet-based [110], SMS text messaging [107], or computer-delivered interventions [112].

The effects did seem to decline in the long run, and the 2 meta-analyses that explicitly examined effectiveness 1 year postintervention (for internet-based and computer-delivered interventions) found that the effect size declined, although it remained statistically significant $[102,109]$.

\section{Adherence (Considered for All Behavioral Areas)}

Typically, adherence data were not reported. Where reported, there were generally decreases in program usage over the intervention period [75]. Rates of attrition were variable and sometimes very high, for instance, in one diet review, it was $0 \%$ to $84 \%$ [61] and in 2 smoking reviews it was $\geq 60 \%$ [86] and $35 \%$ to $84 \%$ (median 70\%) [93]. Bucking the trend, a couple of reviews reported high adherence to digital physical activity interventions [46,60]. Many reviews found a dose-response relationship, whereby the effectiveness of the intervention was positively associated with dietary usage [20,21,61], weight loss [47], smoking [87,90,93,103], and alcohol [79]. However, there was no unanimity, for instance, one combination review found that the attrition rate was lower in internet-based interventions than in face-to-face settings [103] and 2 others found no evidence that retention influenced outcomes [107,110].

\section{Discussion}

\section{Principal Findings}

We reviewed 94 systematic reviews and meta-analyses that examined the effectiveness of digital interventions in changing health-related behavior and improving health outcomes in the areas of diet, physical activity, diet and physical activity combined, alcohol consumption, and smoking cessation, alone or in any other combination. The effectiveness of digital interventions differed according to the area of health behavior reviewed. Small positive effects were evident in smoking- and alcohol-related interventions. Similar findings were observed in the combined diet and physical activity interventions, as well as in other outcome combinations. However, there was little evidence of the effectiveness of stand-alone diet interventions, and evidence of the effectiveness of physical activity interventions was mixed, with some consistently positive evidence for mobile interventions and some promising evidence for exergaming. Digital interventions were most effective in the short-to-mid term (approximately 3 to 6 months), but there was insufficient evidence about their long-term effect. Typically, they were more effective than no intervention. There is mixed evidence on their effectiveness compared with nondigital interventions.

Our secondary objective was to identify differences in effectiveness between the modes of delivery of digital interventions. We identified internet-based interventions to be one of the more effective interventions for each area studied, except for physical activity alone. Mobile interventions were particularly effective for diet and physical activity combined (medium effects), but they were also effective for alcohol and smoking (small effects) and physical activity alone. Social media interventions were not effective for diet and physical activity combined (weight loss interventions), they had mixed effects for diet, and there was limited evidence for other areas. Computer-delivered technologies had small effects for smoking and alcohol consumption, but the effects for diet and physical activity were mixed.

The effect sizes reported in the reviews were generally below the National Institute for Health Care Excellence (NICE) guidelines for effectiveness of interventions, although it was often difficult to compare results with NICE guidelines, as different measures were used.

For weight management, NICE guideline PH53 (Recommendation 13) states that commissioned lifestyle weight management programs should have at least a $60 \%$ completion rate and should be likely to lead to an average weight loss of at least $3 \%$, with at least $30 \%$ of participants losing at least $5 \%$ of their weight [114]. It should be noted that this is based on all participants, that is, those who attend at least one session. For those completing the service, that is, attending at least $75 \%$ of sessions, the key performance indicator of $50 \%$ losing at least $5 \%$ has been set [115]. In contrast, most reviews reported changes in weight in kilograms or changes in BMI, rather than percentage weight loss. The highest effect sizes for BMI in our review were $-0.92 \mathrm{~kg} / \mathrm{m}^{2}$ [49] and $-0.43 \mathrm{~kg} / \mathrm{m}^{2}$ [38], which are extremely unlikely to represent a $3 \%$ or $5 \%$ weight loss in individuals with overweight or obesity. The largest changes in weight found in the reviews of digital interventions were -2.71 $\mathrm{kg}$ in one study [72] and $-2.56 \mathrm{~kg}$ in one meta-analysis [71]. In comparison, the Hartman-Boyce evidence review that supports NICE guideline PH53 found an average effect size of $-2.59 \mathrm{~kg}$ for face-to-face services at 12 months (intention-to-treat analysis) [116]. Most effect sizes from digital interventions did not reach the effect size of face-to-face services.

For smoking, the national outcome measure for stop smoking services is 4-week quits. Smokers attempting to stop without 
additional support generally have a success rate of $25 \%$ at 4 weeks for carbon monoxide-validated quits and a success rate of about $35 \%$ at 4 weeks for self-reported quits [117]. Therefore, to show an impact, services must achieve success rates equivalent to or in excess of these rates that smokers achieve without support. Patients who receive stop smoking service support (behavioral support and pharmacotherapy) are 3 times more likely to quit than those with no support [118], and there is a cessation rate of $35 \%$ for brief intervention services but only at the 4-week point [117]. It is difficult to compare the results of our review with these services because the outcomes of digital smoking interventions are often expressed as ORs for smoking cessation rather than cessation rates. The only available review that used cessation rates demonstrated a cessation rate of $14.8 \%$ [92]. This is lower than the observed rate from stop smoking services or brief advice, but the follow-up point was considerably later and cessation rates decreased over time.

For alcohol, the average weekly reduction in drinking from brief advice interventions is $20 \mathrm{~g}$ of alcohol (about $2.5 \mathrm{UK}$ units) [119], so the reductions in weekly alcohol consumption achieved by digital alcohol interventions are comparable when including all interventions (22 $\mathrm{g}$ of alcohol or $3 \mathrm{UK}$ units) but lower if one restricts attention to high-quality evidence ( $11 \mathrm{~g}$ of alcohol or 1.5 UK units) [77].

To the extent that the effectiveness of digital interventions is below the NICE guidelines, doctors and organizations should be cautious about recommending them to patients who would benefit from behavior change in the 4 areas of our review. However, it may be valuable to recommend them to people who refuse a face-to-face intervention. On the basis of the evidence that we found, digital interventions for weight loss should combine diet and exercise unless they are mobile interventions targeting physical activity.

For digital interventions for both smoking and combined diet and physical activity, there were studies showing significant health effects but not significant behavioral effects. This seems paradoxical. However, it is possible that even a small increase in physical activity or a small improvement in diet, which may not be statistically significant, can improve health markers over an intervention period, particularly in the most inactive and less fit individuals. Light physical activity is beneficial for health outcomes, including cardiometabolic risk factors [120]. We also noted that, although statistically significant, the health effects are small and possibly not meaningful.

\section{Limitations}

Owing to the rapid nature of our review, we did not perform full hand searches or consult experts. This may mean that we overlooked some reviews. At the other end of the spectrum, by reviewing reviews, there is the possibility that some studies were double counted if they were covered in more than one review.

Our ability to make inferences from the literature reviewed is limited for the following reasons.

Heterogeneity was consistently high across reviews. Heterogeneity of effects probably reflects heterogeneity of interventions, which could be a consequence of rapid advances in digital devices and systems. There were also heterogeneous outcome measures. As the reviews covered different types of digital interventions and outcome measures, it was difficult to make comparisons. Differing outcome measures may have differentially impacted the effectiveness of modes of intervention or the general effectiveness of interventions in the areas we investigated. For instance, smaller effect sizes were reported in studies addressing smoking use than in studies on alcohol consumption, possibly because studies addressing alcohol use tended to use reductions in drinking behavior as their outcome variable, while studies addressing smoking use tended to apply the more stringent standard of abstinence [112].

Follow-up times were relatively short, so we cannot know if behavior change would be sustained in the long term. Some trials only provided behavioral data, so we cannot be sure of health outcomes. A review of physical activity found that the average rate of sustained use of digital health interventions over 10 weeks was 50\% [64]. This is consistent with the findings of another systematic review on physical activity apps, which concluded that apps are effective in the short term (up to 3 months) but not longer [121].

Most trials reported intent-to-treat analysis, and typically, adherence data were not reported. This makes it difficult to assess nonsignificant effects to determine whether they resulted from ineffectiveness of treatment or from nonadherence. Where attrition rates were reported, they were often high.

Anecdotally, digital interventions are being used both to supplement and replace face-to-face services. However, most reviews did not discriminate between these functions. In the domain of weight loss, 5 reviews reported enhanced effects on weight loss in interventions that incorporated personal contact or counseling [51,54,57,62,74]. One meta-analysis showed that infrequent in-person treatment was superior in limiting weight gain to computer-based interventions (mean difference $0.5 \mathrm{~kg}$ ). Digital interventions that particularly benefit from involving people alongside are thought to include sensitive tailoring of feedback [57,62] and social support [54].

Confidence ratings were critically low in 79 of 93 reviews $(85 \%)$. However, when isolated, those reviews that were rated critically low presented findings that were consistent with the overall findings: equivocal evidence on effectiveness for diet or physical activity outcomes but consistent findings of short-term effects for alcohol, smoking, and other combined outcomes.

Even when the AMSTAR-2 [16] ratings were moderated (so that justifying any publication language inclusion criterion and providing a list of justifications for excluding reviews were no longer considered critical flaws), 74 of 93 (80\%) reviews were rated critically low. During the generation of confidence ratings, it was noted that many reviews failed to satisfy items 2 and 13. These are considered critical items for all review types. Item 2 specifies that, as a minimum, reviews state that a protocol containing research questions, search strategy, inclusion/exclusion criteria, and a risk of bias assessment was completed before conducting the review. Item 13 dictates that reviews should account for the risk of bias in individual studies when interpreting/discussing the results of the review. The 
inclusion of these items in the AMSTAR-2 rating may represent an aspiration to improve standards. Our AMSTAR-2 quality ratings are consistent with other evidence that suggests that it is possible to satisfy PRISMA standards yet still have poor methodological quality [122]. However, judging reviews according to such high standards, such that they are virtually all rated as being very low, masks the differences in quality.

\section{Future Research Work}

This is the first review of reviews on the effectiveness of digital interventions with such a large scope. It summarizes the state of our knowledge of digital interventions for health improvement behaviors in nonclinical populations. However, the literature could be developed to be more helpful for professionals and organizations who need to decide whether to promote digital interventions, which ones to promote, which areas of behaviors to promote them for, and who to promote them to.

For policy purposes, reviews with mixed controls are of limited use. It matters whether a digital intervention is being compared with no intervention or an active nondigital control. It also matters whether the intervention is a stand-alone digital intervention or whether digital is being used as an adjunct to face-to-face services. We cannot assume that a digital intervention that is successful as an adjunct will also be successful as a stand-alone intervention. Therefore, reviews are needed to separately summarize the evidence base for these different ways of using digital interventions. It could be helpful to have well-structured and coordinated reviews that collate a high-level picture for each area of behavior, which can be updated on a regular basis. We need comparisons with national measures of effectiveness, such as the NICE guidelines, to more easily influence policy.

We also need reviews that can help us determine which interventions are most effective. In the future, it would be helpful to conduct comparative research on the mode of delivery of digital interventions (including comparisons of effect sizes), so that we can determine the most promising interventions for further development. There was also a lack of evidence about the long-term effects of interventions, and more studies on the sustainability of behavior change after digital interventions are needed. It would be especially useful to have this information in comparison with active controls.

Professionals also need to know who should be recommended digital interventions. Therefore, it would also be useful to know whether effect sizes are consistent across various subgroups of the population or whether digital interventions have different effects in different subgroups. We were not specifically looking for this information, but the reviews that were surveyed had mixed findings about whether the effectiveness of digital interventions varied with sex and age. Three meta-analyses found no significant association between sex and effect size or age and effect size $[102,109,110]$. However, one study found that the effect of interventions declined as age increased [105]. There may also be sex-based differences in adherence, and it is plausible, though not proven, that adherence moderates effect size. One narrative synthesis found that women and middle-aged participants were more likely to use web-based intervention services than men and younger participants, and women were more adherent to the overall intervention [103]. There is even less information about differential effects according to socioeconomic status (SES). In the domain of smoking, one review found that the relative effectiveness of technology-based interventions appeared to be comparable between low- and high-SES groups [83].

The acceptability of digital interventions to their target users also warrants further study. In one review of digital interventions of addictive behaviors, participants expressed a preference for internet-based services because of the convenience and increased confidentiality, and individuals who might not otherwise seek treatment said they would consider an internet-based intervention [103].

Providers may be drawn to digital tools in the hope that they are cost-effective. While not the purpose of our review of reviews, we noted insufficient evidence in the reviews to draw any preliminary inferences about the cost-effectiveness of digital interventions. The evidence from the reviews was mixed. There was evidence in favor (internet-based health interventions [41]), evidence against (adaptive e-learning interventions [22]), and mixed evidence: 1 of $3(33 \%)$ web-based interventions was cost-effective compared with in-person interventions at 6 months [52]. Cost-effectiveness may also depend on whether digital interventions supplement or replace face-to-face interventions. Cost-threshold analyses indicated that some form of electronic intervention is likely to be cost-effective when added to nonelectronic behavioral support, but there is substantial uncertainty with regard to determining the most effective (thus most cost-effective) type of electronic intervention, which warrants further research [84]. Future work will need to investigate cost-effectiveness to allocate resources to developing the most promising digital tools.

\section{Conclusions}

Our review of reviews summarizes the state of our knowledge of digital interventions for health improvement behaviors in nonclinical populations. We found positive but small effects for digital interventions that targeted diet and physical activity combined, greater effects—but still small—for smoking and alcohol consumption, and positive, medium-sized effects for mobile interventions for physical activity alone. More high-quality research is needed to assess the sustainability of the effects of digital interventions in the long term, the differences between modes of delivery for digital interventions, their effect on different population subgroups, their cost-effectiveness compared with existing behavior change approaches, and in particular whether they are better used as an adjunct to or replacement for face-to-face treatment. We need the answers to these questions to be able to make an informed decision about whether digital behavior change tools should be integrated into the NHS Health Check program. 


\section{Acknowledgments}

The authors are grateful to Nicola Pearse-Smith for conducting the searches, Rachel Clarke for advice, and Shevez Jeffers for help with the copyediting. This work was funded by Public Health England (PHE). The authors were employed by, or were interns at, PHE when researching this paper. AY was affiliated with the Centre for Diet and Activity Research, MRC Epidemiology Unit, University of Cambridge, when this work was undertaken and is currently affiliated with the Department of Public Health, Environments \& Society, Faculty of Public Health \& Policy, London School of Hygiene \& Tropical Medicine.

\section{Conflicts of Interest}

None declared.

\section{Multimedia Appendix 1}

PRISMA (Preferred Reporting Items for Systematic Reviews and Meta-Analyses) checklist.

[DOC File, 63 KB-Multimedia Appendix 1]

\section{Multimedia Appendix 2}

Search strategy.

[DOCX File, $20 \mathrm{~KB}-$ Multimedia Appendix 2]

\section{Multimedia Appendix 3}

Data extraction form.

[DOC File, 98 KB-Multimedia Appendix 3]

\section{Multimedia Appendix 4}

List of excluded reviews.

[XLSX File (Microsoft Excel File), 20 KB-Multimedia Appendix 4]

\section{Multimedia Appendix 5}

Breakdown of the reviews included in the study.

[DOCX File, 16 KB-Multimedia Appendix 5]

\section{Multimedia Appendix 6}

Key characteristics of the included studies.

[XLSX File (Microsoft Excel File), 43 KB-Multimedia Appendix 6]

\section{References}

1. UK Factsheet. British Heart Foundation. 2021 Mar. URL: https://www.bhf.org.uk/-/media/files/research/heart-statistics/ bhf-cvd-statistics-uk-factsheet.pdf [accessed 2021-03-30]

2. The NHS Long Term Plan. National Health Service Long Term Plan. 2019 Jan 07. URL: https://www.longtermplan.nhs.uk/ wp-content/uploads/2019/08/nhs-long-term-plan-version-1.2.pdf [accessed 2021-03-30]

3. Prevention is better than cure: our vision to help you live well for longer. Department of Health and Social Care. 2018 Jan 23. URL: https://assets.publishing.service.gov.uk/government/uploads/system/uploads/attachment data/file/753688/ Prevention is better than cure 5-11.pdf [accessed 2021-03-30]

4. Thomas K. NHS Health Check Best Practice Guidance. Public Health England.: Public Health England; 2016. URL: https:/ /www.networks.nhs.uk/nhs-networks/healthcare-science/chief-scientific-officers-16-17-workstreams/ diagnostics-supporting-mental-health-t-f-group/lab-in-a-bag-training-workshops/nhs-health-check-best-practice-guidance [accessed 2021-04-20]

5. Public Health England. NHS Health Check: stocktake and action plan. gov.uk. 2018 Jan 23. URL: https://www.gov.uk/ government/publications/ nhs-health-check-stocktake-and-action-plan\#: :text=This\%20stocktake\%20and\%20action\%20plan,in\%20the\%20next\%205\%20years [accessed 2021-03-30]

6. Clifton PM, Keogh JB. Effects of different weight loss approaches on CVD risk. Curr Atheroscler Rep 2018 Apr 25;20(6):1-8. [doi: 10.1007/s11883-018-0728-8]

7. Physical Activity, Health Improvement and Protection. Start Active, Stay Active: A report on physical activity from the four home countries' Chief Medical Officers. Department of Health. 2011 Jul 11. URL: https://assets. 
publishing.service.gov.uk/government/uploads/system/uploads/attachment_data/file/830943/withdrawn_dh_128210.pdf [accessed 2021-03-30]

8. World Health Organisation. Fact sheet about health benefits of smoking cessation. World Health Organisation. 2019. URL: https://www.who.int/tobacco/quitting/benefits/en/ [accessed 2021-03-30]

9. Holmes M, Dale CE, Zuccolo L, Silverwood RJ, Guo Y, Ye Z, InterAct Consortium. Association between alcohol and cardiovascular disease: Mendelian randomisation analysis based on individual participant data. Br Med J 2014 Jul 10;349:g4164 [FREE Full text] [doi: 10.1136/bmj.g4164] [Medline: 25011450]

10. Hollands GJ, French DP, Griffin SJ, Prevost AT, Sutton S, King S, et al. The impact of communicating genetic risks of disease on risk-reducing health behaviour: systematic review with meta-analysis. Br Med J 2016 Mar 15;352:1-11. [doi: 10.1136/bmj.i1102]

11. Shefer G, Silarova B, Usher-Smith J, Griffin S. The response to receiving phenotypic and genetic coronary heart disease risk scores and lifestyle advice - a qualitative study. BMC Public Health 2016 Dec 3;16(1):1-11. [doi: 10.1186/s12889-016-3867-2]

12. Silarova B, Lucas J, Butterworth AS, Di Angelantonio E, Girling C, Lawrence K, et al. Information and Risk Modification Trial (INFORM): design of a randomised controlled trial of communicating different types of information about coronary heart disease risk, alongside lifestyle advice, to achieve change in health-related behaviour. BMC Public Health 2015 Sep 07;15:868 [FREE Full text] [doi: 10.1186/s12889-015-2192-5] [Medline: 26345710]

13. Silarova B, Sharp S, Usher-Smith JA, Lucas J, Payne RA, Shefer G, et al. Effect of communicating phenotypic and genetic risk of coronary heart disease alongside web-based lifestyle advice: the INFORM Randomised Controlled Trial. Heart 2019 Jul;105(13):982-989. [doi: 10.1136/heartjnl-2018-314211] [Medline: 30928969]

14. Murray E, Hekler EB, Andersson G, Collins L, Doherty A, Hollis C, et al. Evaluating digital health interventions: key questions and approaches. Am J Prev Med 2016 Nov;51(5):843-851 [FREE Full text] [doi: 10.1016/j.amepre.2016.06.008] [Medline: 27745684]

15. Moher D, Liberati A, Tetzlaff J, Altman DG, PRISMA Group. Preferred reporting items for systematic reviews and meta-analyses: the PRISMA statement. Ann Intern Med 2009 Aug 18;151(4):264-9, W64. [doi: 10.7326/0003-4819-151-4-200908180-00135] [Medline: 19622511]

16. Shea BJ, Reeves BC, Wells G, Thuku M, Hamel C, Moran J, et al. AMSTAR 2: a critical appraisal tool for systematic reviews that include randomised or non-randomised studies of healthcare interventions, or both. Br Med J 2017 Sep 21;358:j4008 [FREE Full text] [doi: 10.1136/bmj.j4008] [Medline: 28935701]

17. Carey R. A taxonomy of modes of delivery of behaviour change interventions: development and evaluation. In: The European Health Psychologist. 2017 Dec 31 Presented at: European Health Psychology Society Conference; 2017; Padova, Italy p. 666-666 URL: https://osf.io/d4qyk/

18. Cohen J. Statistical Power Analysis for the Behavioral Sciences. New York: Routledge; 1988:1-546.

19. Olivier J, May W, Bell M. Relative effect sizes for measures of risk. Commun Stat Theory Methods 2017 Mar 23;46(14):6774-6781. [doi: 10.1080/03610926.2015.1134575]

20. Burke LE, Wang J, Sevick MA. Self-monitoring in weight loss: a systematic review of the literature. J Am Diet Assoc 2011 Jan;111(1):92-102 [FREE Full text] [doi: 10.1016/j.jada.2010.10.008] [Medline: 21185970]

21. DiFilippo KN, Huang W, Andrade JE, Chapman-Novakofski KM. The use of mobile apps to improve nutrition outcomes: a systematic literature review. J Telemed Telecare 2015 Jul;21(5):243-253. [doi: 10.1177/1357633X15572203] [Medline: 25680388]

22. Harris J, Felix L, Miners A, Murray E, Michie S, Ferguson E, et al. Adaptive e-learning to improve dietary behaviour: a systematic review and cost-effectiveness analysis. Health Technol Assess 2011 Oct;15(37):1-160 [FREE Full text] [doi: 10.3310/hta15370] [Medline: 22030014]

23. McCarroll R, Eyles H, Ni Mhurchu C. Effectiveness of mobile health (mHealth) interventions for promoting healthy eating in adults: A systematic review. Prev Med 2017 Dec;105:156-168. [doi: 10.1016/j.ypmed.2017.08.022] [Medline: 28882743]

24. Bock C, Jarczok MN, Litaker D. Community-based efforts to promote physical activity: a systematic review of interventions considering mode of delivery, study quality and population subgroups. J Sci Med Sport 2014 May;17(3):276-282. [doi: 10.1016/j.jsams.2013.04.009] [Medline: 23693030]

25. Bort-Roig J, Gilson ND, Puig-Ribera A, Contreras RS, Trost SG. Measuring and influencing physical activity with smartphone technology: a systematic review. Sports Med 2014 May;44(5):671-686. [doi: 10.1007/s40279-014-0142-5] [Medline: 24497157]

26. Bottorff JL, Seaton CL, Johnson ST, Caperchione CM, Oliffe JL, More K, et al. An updated review of interventions that include promotion of physical activity for adult men. Sports Med 2015 Jun;45(6):775-800 [FREE Full text] [doi: 10.1007/s40279-014-0286-3] [Medline: 25430599]

27. Buchholz SW, Wilbur J, Ingram D, Fogg L. Physical activity text messaging interventions in adults: a systematic review. Worldviews Evid Based Nurs 2013 Aug;10(3):163-173. [doi: 10.1111/wvn.12002] [Medline: 23746267]

28. Cheatham S, Stull KR, Fantigrassi M, Motel I. The efficacy of wearable activity tracking technology as part of a weight loss program: a systematic review. J Sports Med Phys Fitness 2018 Apr;58(4):534-548. [doi:

10.23736/S0022-4707.17.07437-0] [Medline: 28488834] 
29. Davies CA, Spence JC, Vandelanotte C, Caperchione CM, Mummery W. Meta-analysis of internet-delivered interventions to increase physical activity levels. Int J Behav Nutr Phys Act 2012 Apr 30;9:52 [FREE Full text] [doi: 10.1186/1479-5868-9-52] [Medline: 22546283]

30. Direito A, Carraça E, Rawstorn J, Whittaker R, Maddison R. mHealth technologies to influence physical activity and sedentary behaviors: behavior change techniques, systematic review and meta-analysis of randomized controlled trials. Ann Behav Med 2017 Apr;51(2):226-239. [doi: 10.1007/s12160-016-9846-0] [Medline: 27757789]

31. Elavsky S, Knapova L, Klocek A, Smahel D. Mobile health interventions for physical activity, sedentary behavior, and sleep in adults aged 50 years and older: a systematic literature review. J Aging Phys Act 2019 Aug 01;27(4):565-593. [doi: 10.1123/japa.2017-0410] [Medline: 30507266]

32. Freak-Poli R, Cumpston M, Peeters A, Clemes SA. Workplace pedometer interventions for increasing physical activity. Cochrane Database Syst Rev 2013 Apr 30(4):CD009209. [doi: 10.1002/14651858.CD009209.pub2] [Medline: 23633368]

33. George ES, Kolt GS, Duncan MJ, Caperchione CM, Mummery WK, Vandelanotte C, et al. A review of the effectiveness of physical activity interventions for adult males. Sports Med 2012 Apr 01;42(4):281-300. [doi: 10.2165/11597220-000000000-00000] [Medline: 22350570]

34. Hakala S, Rintala A, Immonen J, Karvanen J, Heinonen A, Sjögren T. Effectiveness of physical activity promoting technology-based distance interventions compared to usual care. Systematic review, meta-analysis and meta-regression. Eur J Phys Rehabil Med 2017 Dec;53(6):953-967. [doi: 10.23736/S1973-9087.17.04585-3] [Medline: 28466628]

35. Jahangiry L, Farhangi MA, Shab-Bidar S, Rezaei F, Pashaei T. Web-based physical activity interventions: a systematic review and meta-analysis of randomized controlled trials. Public Health 2017 Nov;152:36-46. [doi: 10.1016/j.puhe.2017.06.005] [Medline: 28734170]

36. Jenkins A, Christensen H, Walker JG, Dear K. The effectiveness of distance interventions for increasing physical activity: a review. Am J Health Promot 2009;24(2):102-117. [doi: 10.4278/ajhp.0801158] [Medline: 19928483]

37. Johnson D, Deterding S, Kuhn K, Staneva A, Stoyanov S, Hides L. Gamification for health and wellbeing: a systematic review of the literature. Internet Interv 2016 Nov;6:89-106 [FREE Full text] [doi: 10.1016/j.invent.2016.10.002] [Medline: 30135818]

38. Flores Mateo G, Granado-Font E, Ferré-Grau C, Montaña-Carreras X. Mobile phone apps to promote weight loss and increase physical activity: a systematic review and meta-analysis. J Med Internet Res 2015 Nov 10;17(11):e253 [FREE Full text] [doi: 10.2196/jmir.4836] [Medline: 26554314]

39. Muellmann S, Forberger S, Möllers T, Bröring E, Zeeb H, Pischke CR. Effectiveness of eHealth interventions for the promotion of physical activity in older adults: a systematic review. Prev Med 2018 Mar;108:93-110. [doi: 10.1016/j.ypmed.2017.12.026] [Medline: 29289643]

40. Müller AM, Khoo S. Non-face-to-face physical activity interventions in older adults: a systematic review. Int J Behav Nutr Phys Act 2014 Mar 10;11(1):35 [FREE Full text] [doi: 10.1186/1479-5868-11-35] [Medline: 24612748]

41. Muntaner A, Vidal-Conti J, Palou P. Increasing physical activity through mobile device interventions: a systematic review. Health Informatics J 2016 Sep;22(3):451-469. [doi: 10.1177/1460458214567004] [Medline: 25649783]

42. O'Reilly GA, Spruijt-Metz D. Current mHealth technologies for physical activity assessment and promotion. Am J Prev Med 2013 Oct;45(4):501-507 [FREE Full text] [doi: 10.1016/j.amepre.2013.05.012] [Medline: 24050427]

43. Peng W, Lin J, Crouse J. Is playing exergames really exercising? A meta-analysis of energy expenditure in active video games. Cyberpsychol Behav Soc Netw 2011 Nov;14(11):681-688. [doi: 10.1089/cyber.2010.0578] [Medline: 21668370]

44. Song Y. Feasibility and effectiveness of mobile phones in physical activity promotion for adults 50 years and older: a systematic review. Top Geriatr Rehabil 2018;34(3):A-222. [doi: 10.1097/tgr.0000000000000197]

45. Stephenson A, McDonough SM, Murphy MH, Nugent CD, Mair JL. Using computer, mobile and wearable technology enhanced interventions to reduce sedentary behaviour: a systematic review and meta-analysis. Int J Behav Nutr Phys Act 2017 Aug 11;14(1):105 [FREE Full text] [doi: 10.1186/s12966-017-0561-4] [Medline: 28800736]

46. Street TD, Lacey SJ, Langdon RR. Gaming your way to health: a systematic review of exergaming programs to increase health and exercise behaviors in adults. Games Health J 2017 Jun;6(3):136-146. [doi: 10.1089/g4h.2016.0102] [Medline: $\underline{28448175]}$

47. Aalbers T, Baars M, Rikkert MO. Characteristics of effective internet-mediated interventions to change lifestyle in people aged 50 and older: a systematic review. Ageing Res Rev 2011 Sep;10(4):487-497. [doi: 10.1016/j.arr.2011.05.001] [Medline: $\underline{21628005}]$

48. Allen JK, Stephens J, Patel A. Technology-assisted weight management interventions: systematic review of clinical trials. Telemed J E Health 2014 Dec;20(12):1103-1120 [FREE Full text] [doi: 10.1089/tmj.2014.0030] [Medline: 25409001]

49. An R, Ji M, Zhang S. Effectiveness of social media-based interventions on weight-related behaviors and body weight status: review and meta-analysis. Am J Health Behav 2017 Nov 01;41(6):670-682. [doi: 10.5993/AJHB.41.6.1] [Medline: 29025495]

50. Bacigalupo R, Cudd P, Littlewood C, Bissell P, Hawley MS, Buckley Woods H. Interventions employing mobile technology for overweight and obesity: an early systematic review of randomized controlled trials. Obes Rev 2013 Apr;14(4):279-291 [FREE Full text] [doi: 10.1111/obr.12006] [Medline: 23167478] 
51. Bassi N, Karagodin I, Wang S, Vassallo P, Priyanath A, Massaro E, et al. Lifestyle modification for metabolic syndrome: a systematic review. Am J Med 2014 Dec;127(12):1242.e1-1242.e10. [doi: 10.1016/j.amjmed.2014.06.035] [Medline: $\underline{25004456]}$

52. Beleigoli AM, Andrade AQ, Cançado AG, Paulo MN, De Fátima H Diniz M, Ribeiro AL. Web-Based Digital Health Interventions for Weight Loss and Lifestyle Habit Changes in Overweight and Obese Adults: Systematic Review and Meta-Analysis. J Med Internet Res 2019 Jan 08;21(1):e298 [FREE Full text] [doi: 10.2196/jmir.9609] [Medline: 30622090]

53. Carvalho de Menezes M, Bedeschi LB, Santos LC, Lopes AC. Interventions directed at eating habits and physical activity using the Transtheoretical Model: a systematic review. Nutr Hosp 2016 Sep 20;33(5):586. [doi: 10.20960/nh.586] [Medline: 27759990]

54. Coons MJ, Demott A, Buscemi J, Duncan JM, Pellegrini CA, Steglitz J, et al. Technology interventions to curb obesity: a systematic review of the current literature. Curr Cardiovasc Risk Rep 2012 Apr;6(2):120-134 [FREE Full text] [doi: 10.1007/s12170-012-0222-8] [Medline: 23082235]

55. Dutton GR, Laitner MH, Perri MG. Lifestyle interventions for cardiovascular disease risk reduction: a systematic review of the effects of diet composition, food provision, and treatment modality on weight loss. Curr Atheroscler Rep 2014 Oct;16(10):442 [FREE Full text] [doi: 10.1007/s11883-014-0442-0] [Medline: 25092578]

56. Elaheebocus SM, Weal M, Morrison L, Yardley L. Peer-based social media features in behavior change interventions: systematic review. J Med Internet Res 2018 Feb 22;20(2):e20 [FREE Full text] [doi: 10.2196/jmir.8342] [Medline: 29472174]

57. Fry JP, Neff RA. Periodic prompts and reminders in health promotion and health behavior interventions: systematic review. J Med Internet Res 2009 May 14;11(2):e16 [FREE Full text] [doi: 10.2196/jmir.1138] [Medline: 19632970]

58. Kodama S, Saito K, Tanaka S, Horikawa C, Fujiwara K, Hirasawa R, et al. Effect of web-based lifestyle modification on weight control: a meta-analysis. Int J Obes (Lond) 2012 May;36(5):675-685. [doi: 10.1038/ijo.2011.121] [Medline: 21694698]

59. Liu F, Kong X, Cao J, Chen S, Li C, Huang J, et al. Mobile phone intervention and weight loss among overweight and obese adults: a meta-analysis of randomized controlled trials. Am J Epidemiol 2015 Mar 01;181(5):337-348 [FREE Full text] [doi: 10.1093/aje/kwu260] [Medline: 25673817]

60. Lyzwinski L. A systematic review and meta-analysis of mobile devices and weight loss with an intervention content analysis. J Personalized Med 2014 Jun 30;4(3):311-385. [doi: 10.3390/jpm4030311] [Medline: 25563356]

61. Maher CA, Lewis LK, Ferrar K, Marshall S, De Bourdeaudhuij I, Vandelanotte C. Are health behavior change interventions that use online social networks effective? A systematic review. J Med Internet Res 2014 Feb 14;16(2):e40 [FREE Full text] [doi: 10.2196/jmir.2952] [Medline: 24550083]

62. Manzoni GM, Pagnini F, Corti S, Molinari E, Castelnuovo G. Internet-based behavioral interventions for obesity: an updated systematic review. Clin Pract Epidemiol Ment Health 2011 Mar 04;7:19-28 [FREE Full text] [doi: 10.2174/1745017901107010019] [Medline: 21552423]

63. Maon S, Edirippulige S, Ware R, Batch J. The use of web-based interventions to prevent excessive weight gain. J Telemed Telecare 2012 Jan;18(1):37-41. [doi: 10.1258/jtt.2011.110306] [Medline: 22101608]

64. Maxwell K. Technology as a Health Intervention and the Self-Efficacy of Men. Walden University. 2015. URL: https:/ /scholarworks.waldenu.edu/dissertations/418/ [accessed 2021-04-20]

65. Mita G, Ni Mhurchu C, Jull A. Effectiveness of social media in reducing risk factors for noncommunicable diseases: a systematic review and meta-analysis of randomized controlled trials. Nutr Rev 2016 Apr;74(4):237-247 [FREE Full text] [doi: 10.1093/nutrit/nuv106] [Medline: 26946250]

66. Podina IR, Fodor LA. Critical review and meta-analysis of multicomponent behavioral e-health interventions for weight loss. Health Psychol 2018 Jun;37(6):501-515. [doi: 10.1037/hea0000623] [Medline: 29733617]

67. Ryan K, Dockray S, Linehan C. A systematic review of tailored eHealth interventions for weight loss. Digit Health 2019;5:2055207619826685 [FREE Full text] [doi: 10.1177/2055207619826685] [Medline: 30783535]

68. Schoeppe S, Alley S, Van Lippevelde W, Bray NA, Williams SL, Duncan MJ, et al. Efficacy of interventions that use apps to improve diet, physical activity and sedentary behaviour: a systematic review. Int J Behav Nutr Phys Act 2016 Dec 07;13(1):127 [FREE Full text] [doi: 10.1186/s12966-016-0454-y] [Medline: 27927218]

69. Seo D, Niu J. Evaluation of internet-based interventions on waist circumference reduction: a meta-analysis. J Med Internet Res 2015 Jul 21;17(7):e181 [FREE Full text] [doi: 10.2196/jmir.3921] [Medline: 26199208]

70. Sherrington A, Newham JJ, Bell R, Adamson A, McColl E, Araujo-Soares V. Systematic review and meta-analysis of internet-delivered interventions providing personalized feedback for weight loss in overweight and obese adults. Obes Rev 2016 Jun;17(6):541-551 [FREE Full text] [doi: 10.1111/obr.12396] [Medline: 26948257]

71. Siopis G, Chey T, Allman-Farinelli M. A systematic review and meta-analysis of interventions for weight management using text messaging. J Hum Nutr Diet 2015 Feb;28 Suppl 2:1-15. [doi: 10.1111/jhn.12207] [Medline: 24480032]

72. Tang JC, Abraham C, Greaves CJ, Nikolaou V. Self-directed interventions to promote weight loss: a systematic review and meta-analysis. Health Psychol Rev 2016 May 10;10(3):358-372. [doi: 10.1080/17437199.2016.1172979]

73. Tsoli S, Sutton S, Kassavou A. Interactive voice response interventions targeting behaviour change: a systematic literature review with meta-analysis and meta-regression. BMJ Open 2018 Feb 24;8(2):e018974. [doi: 10.1136/bmjopen-2017-018974] 
74. Wieland L, Falzon L, Sciamanna CN, Trudeau KJ, Brodney S, Schwartz JE, et al. Interactive computer-based interventions for weight loss or weight maintenance in overweight or obese people. Cochrane Database Syst Rev 2012 Aug 15(8):CD007675 [FREE Full text] [doi: 10.1002/14651858.CD007675.pub2] [Medline: 22895964]

75. Williams G, Hamm MP, Shulhan J, Vandermeer B, Hartling L. Social media interventions for diet and exercise behaviours: a systematic review and meta-analysis of randomised controlled trials. BMJ Open 2014 Feb 12;4(2):e003926 [FREE Full text] [doi: 10.1136/bmjopen-2013-003926] [Medline: 24525388]

76. Willis EA, Szabo-Reed AN, Ptomey LT, Steger FL, Honas JJ, Washburn RA, et al. Do weight management interventions delivered by online social networks effectively improve body weight, body composition, and chronic disease risk factors? A systematic review. J Telemed Telecare 2017 Feb;23(2):263-272 [FREE Full text] [doi: 10.1177/1357633X16630846] [Medline: 26880695]

77. Kaner E, Beyer FR, Garnett C, Crane D, Brown J, Muirhead C, et al. Personalised digital interventions for reducing hazardous and harmful alcohol consumption in community-dwelling populations. Cochrane Database Syst Rev 2017 Sep 25;9:CD011479 [FREE Full text] [doi: 10.1002/14651858.CD011479.pub2] [Medline: 28944453]

78. Kolar C, von Treuer K. Alcohol misuse interventions in the workplace: a systematic review of workplace and sports management alcohol interventions. Int J Ment Health Addiction 2015 Jun 20;13(5):563-583. [doi: 10.1007/s11469-015-9558-x]

79. Riper H, Spek V, Boon B, Conijn B, Kramer J, Martin-Abello K, et al. Effectiveness of E-self-help interventions for curbing adult problem drinking: a meta-analysis. J Med Internet Res 2011 Jun 30;13(2):e42 [FREE Full text] [doi: 10.2196/jmir.1691] [Medline: 21719411]

80. Riper H, Blankers M, Hadiwijaya H, Cunningham J, Clarke S, Wiers R, et al. Effectiveness of guided and unguided low-intensity internet interventions for adult alcohol misuse: a meta-analysis. PLoS One 2014;9(6):e99912 [FREE Full text] [doi: 10.1371/journal.pone.0099912] [Medline: 24937483]

81. Vernon ML. A review of computer-based alcohol problem services designed for the general public. J Subst Abuse Treat 2010 Apr;38(3):203-211 [FREE Full text] [doi: 10.1016/j.jsat.2009.11.001] [Medline: 20015607]

82. Black N, Mullan B, Sharpe L. Computer-delivered interventions for reducing alcohol consumption: meta-analysis and meta-regression using behaviour change techniques and theory. Health Psychol Rev 2016 Sep;10(3):341-357. [doi: 10.1080/17437199.2016.1168268] [Medline: 26999311]

83. Boland V, Stockings EA, Mattick RP, McRobbie H, Brown J, Courtney RJ. The methodological quality and effectiveness of technology-based smoking cessation interventions for disadvantaged groups: a systematic review and meta-analysis. Nicotine Tob Res 2018 Feb 07;20(3):276-285. [doi: 10.1093/ntr/ntw391] [Medline: 28034998]

84. Chen Y, Madan J, Welton N, Yahaya I, Aveyard P, Bauld L, et al. Effectiveness and cost-effectiveness of computer and other electronic aids for smoking cessation: a systematic review and network meta-analysis. Health Technol Assess 2012 Oct;16(38):1-205, iii [FREE Full text] [doi: 10.3310/hta16380] [Medline: 23046909]

85. Cheung KL, Wijnen B, de Vries H. A review of the theoretical basis, effects, and cost effectiveness of online smoking cessation interventions in the netherlands: a mixed-methods approach. J Med Internet Res 2017 Jun 23;19(6):e230 [FREE Full text] [doi: 10.2196/jmir.7209] [Medline: 28645889]

86. Danielsson A, Eriksson A, Allebeck P. Technology-based support via telephone or web: a systematic review of the effects on smoking, alcohol use and gambling. Addict Behav 2014 Dec;39(12):1846-1868. [doi: 10.1016/j.addbeh.2014.06.007] [Medline: 25128637]

87. Gainsbury S, Blaszczynski A. A systematic review of Internet-based therapy for the treatment of addictions. Clin Psychol Rev 2011 Apr;31(3):490-498. [doi: 10.1016/j.cpr.2010.11.007] [Medline: 21146272]

88. Graham A, Carpenter K, Cha S, Cole S, Jacobs M, Raskob M, et al. Systematic review and meta-analysis of internet interventions for smoking cessation among adults. Subst Abuse Rehabil 2016;7:55-69 [FREE Full text] [doi: 10.2147/SAR.S101660] [Medline: 27274333]

89. Health Information and Quality Authority. Health technology assessment (HTA) of smoking cessation interventions. Health Information and Quality Authority. 2017. URL: https://www.hiqa.ie/sites/default/files/2017-04/

Smoking\%20Cessation\%20HTA.pdf [accessed 2021-03-30]

90. Hutton HE, Wilson LM, Apelberg BJ, Tang EA, Odelola O, Bass EB, et al. A systematic review of randomized controlled trials: web-based interventions for smoking cessation among adolescents, college students, and adults. Nicotine Tob Res 2011 Apr;13(4):227-238. [doi: 10.1093/ntr/ntq252] [Medline: 21350042]

91. McCrabb S, Baker AL, Attia J, Skelton E, Twyman L, Palazzi K, et al. Internet-based programs incorporating behavior change techniques are associated with increased smoking cessation in the general population: a systematic review and meta-analysis. Ann Behav Med 2019 Feb 01;53(2):180-195. [doi: 10.1093/abm/kay026] [Medline: 29750240]

92. Myung S, McDonnell DD, Kazinets G, Seo HG, Moskowitz JM. Effects of web- and computer-based smoking cessation programs: meta-analysis of randomized controlled trials. Arch Intern Med 2009 May 25;169(10):929-937. [doi: 10.1001/archinternmed.2009.109] [Medline: 19468084]

93. Naslund JA, Kim SJ, Aschbrenner KA, McCulloch LJ, Brunette MF, Dallery J, et al. Systematic review of social media interventions for smoking cessation. Addict Behav 2017 Oct;73:81-93 [FREE Full text] [doi: 10.1016/j.addbeh.2017.05.002] [Medline: 28499259] 
94. Scott-Sheldon LA, Lantini R, Jennings EG, Thind H, Rosen RK, Salmoirago-Blotcher E, et al. Text messaging-based interventions for smoking cessation: a systematic review and meta-analysis. JMIR Mhealth Uhealth 2016 May 20;4(2):e49 [FREE Full text] [doi: 10.2196/mhealth.5436] [Medline: 27207211]

95. Shahab L, McEwen A. Online support for smoking cessation: a systematic review of the literature. Addiction 2009 Nov;104(11):1792-1804. [doi: 10.1111/j.1360-0443.2009.02710.x] [Medline: $\underline{19832783}$ ]

96. Spohr SA, Nandy R, Gandhiraj D, Vemulapalli A, Anne S, Walters ST. Efficacy of SMS text message interventions for smoking cessation: a meta-analysis. J Subst Abuse Treat 2015 Sep;56:1-10. [doi: 10.1016/j.jsat.2015.01.011] [Medline: 25720333]

97. Taylor G, Dalili MN, Semwal M, Civljak M, Sheikh A, Car J. Internet-based interventions for smoking cessation. Cochrane Database Syst Rev 2017 Sep 04;9:CD007078 [FREE Full text] [doi: 10.1002/14651858.CD007078.pub5] [Medline: 28869775]

98. Whittaker R, McRobbie H, Bullen C, Rodgers A, Gu Y. Mobile phone-based interventions for smoking cessation. Cochrane Database Syst Rev 2016 Apr 10;4:CD006611 [FREE Full text] [doi: 10.1002/14651858.CD006611.pub4] [Medline: 27060875]

99. Afshin A, Babalola D, Mclean M, Yu Z, Ma W, Chen C, et al. Information technology and lifestyle: a systematic evaluation of internet and mobile interventions for improving diet, physical activity, obesity, tobacco, and alcohol use. J Am Heart Assoc 2016 Aug 31;5(9):e003058 [FREE Full text] [doi: 10.1161/JAHA.115.003058] [Medline: 27581172]

100. Aneni EC, Roberson LL, Maziak W, Agatston AS, Feldman T, Rouseff M, et al. PLoS One 2014;9(1):e83594 [FREE Full text] [doi: 10.1371/journal.pone.0083594] [Medline: 24421894]

101. Armanasco AA, Miller YD, Fjeldsoe BS, Marshall AL. Preventive health behavior change text message interventions: a meta-analysis. Am J Prev Med 2017 Mar;52(3):391-402. [doi: 10.1016/j.amepre.2016.10.042] [Medline: 28073656]

102. Beishuizen CR, Stephan BC, van Gool WA, Brayne C, Peters RJ, Andrieu S, et al. Web-based interventions targeting cardiovascular risk factors in middle-aged and older people: a systematic review and meta-analysis. J Med Internet Res 2016 Mar 11;18(3):e55 [FREE Full text] [doi: 10.2196/jmir.5218] [Medline: 26968879]

103. Chebli J, Blaszczynski A, Gainsbury SM. Internet-based interventions for addictive behaviours: a systematic review. J Gambl Stud 2016 Dec;32(4):1279-1204. [doi: 10.1007/s10899-016-9599-5] [Medline: 27002522]

104. Covolo L, Ceretti E, Moneda M, Castaldi S, Gelatti U. Does evidence support the use of mobile phone apps as a driver for promoting healthy lifestyles from a public health perspective? A systematic review of Randomized Control Trials. Patient Educ Couns 2017 Dec;100(12):2231-2243. [doi: 10.1016/j.pec.2017.07.032] [Medline: 28855063]

105. Cugelman B, Thelwall M, Dawes P. Online interventions for social marketing health behavior change campaigns: a meta-analysis of psychological architectures and adherence factors. J Med Internet Res 2011 Feb 14;13(1):e17 [FREE Full text] [doi: 10.2196/jmir.1367] [Medline: 21320854]

106. De Leon E, Fuentes LW, Cohen JE. Characterizing periodic messaging interventions across health behaviors and media: systematic review. J Med Internet Res 2014 Mar 25;16(3):e93 [FREE Full text] [doi: 10.2196/jmir.2837] [Medline: 24667840]

107. Head KJ, Noar SM, Iannarino NT, Grant Harrington N. Efficacy of text messaging-based interventions for health promotion: a meta-analysis. Soc Sci Med 2013 Nov;97:41-48. [doi: 10.1016/j.socscimed.2013.08.003] [Medline: 24161087]

108. Hou S, Charlery SR, Roberson K. Systematic literature review of Internet interventions across health behaviors. Health Psychol Behav Med 2014 Jan 01;2(1):455-481 [FREE Full text] [doi: 10.1080/21642850.2014.895368] [Medline: 25750795]

109. Krebs P, Prochaska JO, Rossi JS. A meta-analysis of computer-tailored interventions for health behavior change. Prev Med 2010;51(3-4):214-221 [FREE Full text] [doi: 10.1016/j.ypmed.2010.06.004] [Medline: 20558196]

110. Lustria ML, Noar SM, Cortese J, Van Stee SK, Glueckauf RL, Lee J. A meta-analysis of web-delivered tailored health behavior change interventions. J Health Commun 2013;18(9):1039-1069. [doi: 10.1080/10810730.2013.768727] [Medline: 23750972]

111. Palmer M, Sutherland J, Barnard S, Wynne A, Rezel E, Doel A, et al. The effectiveness of smoking cessation, physical activity/diet and alcohol reduction interventions delivered by mobile phones for the prevention of non-communicable diseases: a systematic review of randomised controlled trials. PLoS One 2018;13(1):e0189801 [FREE Full text] [doi: 10.1371/journal.pone.0189801] [Medline: 29304148]

112. Rooke S, Thorsteinsson E, Karpin A, Copeland J, Allsop D. Computer-delivered interventions for alcohol and tobacco use: a meta-analysis. Addiction 2010 Aug;105(8):1381-1390. [doi: 10.1111/j.1360-0443.2010.02975.x] [Medline: 20528806]

113. Webb TL, Joseph J, Yardley L, Michie S. Using the internet to promote health behavior change: a systematic review and meta-analysis of the impact of theoretical basis, use of behavior change techniques, and mode of delivery on efficacy. J Med Internet Res 2010 Feb 17;12(1):e4 [FREE Full text] [doi: 10.2196/jmir.1376] [Medline: 20164043]

114. Weight management: lifestyle services for overweight or obese adults. National Institute for Health and Care Excellence. 2014. URL: https://www.nice.org.uk/guidance/ph53/resources/ weight-management-lifestyle-services-for-overweight-or-obese-adults-pdf-1996416726469 [accessed 2021-03-30]

115. Thomson L. Key Performance Indicators: Tier 2 Weight Management Services for Adults. Public Health England. 2017. URL: https://assets.publishing.service.gov.uk/government/uploads/system/uploads/attachment data/file/656531/ adult_weight_management_key_performance_indicators.pdf [accessed 2021-03-30] 
116. Hartmann-Boyce J, Aveyard P, Koshiaris C, Jebb SA. Development of tools to study personal weight control strategies: OxFAB taxonomy. Obesity (Silver Spring) 2016 Feb;24(2):314-320 [FREE Full text] [doi: 10.1002/oby.21341] [Medline: 26748902]

117. West R, May S, West M, Croghan E, McEwen A. Performance of English stop smoking services in first 10 years: analysis of service monitoring data. Br Med J 2013 Aug 19;347:f4921 [FREE Full text] [doi: 10.1136/bmj.f4921] [Medline: 23963106]

118. West R, Papadakis S. Stop smoking services: increased chances of quitting. National Centre for Smoking Cessation and Training. 2019. URL: https://www.ncsct.co.uk/usr/pub/Stop\%20smoking\%20services\%20effectiveness.pdf [accessed 2021-03-30]

119. Kaner E, Beyer F, Dickinson HO, Pienaar E, Campbell F, Schlesinger C, et al. Effectiveness of brief alcohol interventions in primary care populations. Cochrane Database Syst Rev 2007 Apr 18(2):CD004148. [doi: 10.1002/14651858.CD004148.pub3] [Medline: 17443541]

120. Amagasa S, Machida M, Fukushima N, Kikuchi H, Takamiya T, Odagiri Y, et al. Is objectively measured light-intensity physical activity associated with health outcomes after adjustment for moderate-to-vigorous physical activity in adults? A systematic review. Int J Behav Nutr Phys Act 2018 Jul 09;15(1):65 [FREE Full text] [doi: 10.1186/s12966-018-0695-z] [Medline: 29986718]

121. Romeo A, Edney S, Plotnikoff R, Curtis R, Ryan J, Sanders I, et al. Can smartphone apps increase physical activity? Systematic review and meta-analysis. J Med Internet Res 2019 Mar 19;21(3):e12053 [FREE Full text] [doi: 10.2196/12053] [Medline: 30888321$]$

122. Johnson BT, Hennessy EA. Systematic reviews and meta-analyses in the health sciences: best practice methods for research syntheses. Soc Sci Med 2019 Jul;233:237-251. [doi: 10.1016/j.socscimed.2019.05.035] [Medline: 31233957]

\author{
Abbreviations \\ CVD: cardiovascular disease \\ NHS: National Health Service \\ NICE: National Institute for Health Care Excellence \\ PHE: Public Health England \\ PRISMA: Preferred Reporting Items for Systematic Reviews and Meta-Analyses \\ RCT: randomized controlled trial \\ RR: risk ratio \\ SES: socioeconomic status \\ SMD: standardized mean difference
}

Edited by R Kukafka; submitted 04.06.20; peer-reviewed by S Hakala, T Stütz; comments to author 14.07.20; revised version received 05.10.20; accepted 03.02.21; published 14.05.21

Please cite as:

Gold N, Yau A, Rigby B, Dyke C, Remfry EA, Chadborn T

Effectiveness of Digital Interventions for Reducing Behavioral Risks of Cardiovascular Disease in Nonclinical Adult Populations: Systematic Review of Reviews

J Med Internet Res 2021;23(5):e19688

URL: https://www.jmir.org/2021/5/e19688

doi: $10.2196 / 19688$

PMID:

CNatalie Gold, Amy Yau, Benjamin Rigby, Chris Dyke, Elizabeth Alice Remfry, Tim Chadborn. Originally published in the Journal of Medical Internet Research (https://www.jmir.org), 14.05.2021. This is an open-access article distributed under the terms of the Creative Commons Attribution License (https://creativecommons.org/licenses/by/4.0/), which permits unrestricted use, distribution, and reproduction in any medium, provided the original work, first published in the Journal of Medical Internet Research, is properly cited. The complete bibliographic information, a link to the original publication on https://www.jmir.org/, as well as this copyright and license information must be included. 